\title{
Fator de Bayes a Posteriori para Comparar os Coeficientes de Modelos de Regressão
}

\author{
Margareth Moreira Cordeiro
}

Orientador: DR. Josemar RodRigues

Dissertação apresentada ao Instituto de Ciências Matemáticas de São Carlos - USP, como parte dos requisitos para obtenção do título de mestre em "Ciências de Computação e Matemática Computacional".

São Carlos 
Ao meu marido Acácio e ao meu filho Luiz Acácio, grandes companheiros e amigos, pelo apoio, compreensão, amor e carinho. 
NÃo conto gozar a MINHa VIDA; NEM EM GOZÁ-LA PENSO.

SÓ QUERO TORNÁ-LA GRANDE, AINDA QUE PARA ISSO TENHA DE SER O MEU CORPO E A (MINHA ALMA) A LENHA DESSE FOGO.

Fernando Pessoa 


\section{AGRADECIMENTOS}

A Deus por mais esta realização em minha vida.

Ao prof. Dr. Josemar Rodrigues, pelo estimulo e apoio desde o meu ingresso no curso de pós-graduação até a concretização deste trabalho, pela felicidade de ter sido sua orientada e poder presenciar tantos exemplos de sabedoria, humildade, disposição e incentivos constantes.

Aos meus pais Alcides e Ruth e sogros Luiz e Therezinha, que souberam entender os momentos de ausência, pelo insentivo e amor.

Aos Professores, Funcionários e Colegas da Pós-Graduação pelo harmonioso convívio, tornando mais felizes o decorrer destes anos.

À CAPES pelo apoio financeiro parcial recebido.

À todas as pessoas que, direta ou inderetamente, contribuíram na realização deste trabalho. 


\section{RESUMO}

Nesta dissertação, abordamos uma análise Bayesiana para comparar os coe-

ficientes de modelos de regressão linear. Esta análise foi baseada no fator de Bayes a posteriori introduzido por Aitkin (1991), considerando-se diferentes restriçôes sobre o modelo adotado e distribuições a priori não informativas. Em todos os modelos adotados observou-se que o fator de Bayes a posteriori não é influenciado pela distribuição a priori e pelo tamanho da amostra (paradoxo de Lindley).

Fez-se um estudo numérico para comparar o fator de Bayes a posteriori e o teste da razão de verossimilhança, concluindo-se que o fator de Bayes a posteriori é mais eficiente.

Também foi proposto um novo critério denominado de teste da razão de entropia a posteriori. Os resultados obtidos através de simulações, quando comparados com o fator de Bayes a posteriori, indicaram que dependendo da escala utilizada o critério proposto é mais eficiente.

Desenvolveu-se ainda uma aplicação do fator de Bayes a posteriori para dados relacionados ao desenvolvimento de um sensor de corrente elétrica utilizando fibras ópticas (Vieira, 1992). 


\section{ABSTRACT}

The Bayesian analysis is covered in this dissertation to compare the coefficients of linear regression models. This analysis was based on the posterior Bayes factor introduced by Aitkin (1991), taking into account different restrictions on the adopted model and non informative priors distributions. On all the adopted models it was noted that the posterior Bayes factor is not influenced by the a prior distribution and by the sample size (Lindley paradox).

A numerical study was made to compare the posterior Bayes factor and the likelihood ratio test. The conclusion was that the posterior Bayes factor is more efficient.

It was also proposed a new criterium called posterior entropy ratio test. The results achieved by means of simulations when compared with the posterior Bayes factor showed that depending on the scale used the proposed criterium is more efficient.

A posterior Bayes factor application was also developed for data related to the development of an electric current sensor by the use of optical fibers (Vieira, 1992). 


\section{Conteúdo}

1 Introdução 1

2 Formulação do Modelo e o Fator de Bayes a Posteriori 7

2.1 Introdução . . . . . . . . . . . . . . . 7

2.2 Variáveis sem Erro $\ldots \ldots \ldots \ldots \ldots \ldots \ldots$

2.3 Variáveis com Erros $\ldots \ldots \ldots \ldots \ldots \ldots \ldots \ldots$

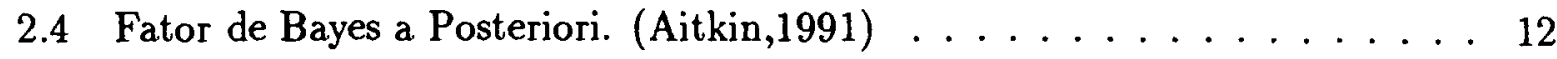

3 Comparação de dois Modelos sem Erro nas Variáveis 14

3.1 Introdução . . . . . . . . . . . . . . . . . 14

3.2 Comparação de dois Modelos de Regressão com Variâncias Iguais e Desconhecidas . . . . . . . . . . . . . . . 15

3.2.1 Comentários Importantes: $\ldots \ldots \ldots \ldots \ldots \ldots \ldots \ldots$ 
3.3 Comparação de dois Modelos de Regressão com Variâncias Desiguais e Desconhecidas . . . . . . . . . . . . . . . . . 23

3.3.1 Comentários Importantes: $\ldots \ldots \ldots \ldots \ldots \ldots$

3.4 Comparação Simultânea dos Coeficientes e Variâncias . . . . . . . . . . 31

3.4 .1 Interpretações $\ldots \ldots \ldots \ldots \ldots \ldots \ldots \ldots \ldots \ldots$

3.5 Ilustração Numérica $\ldots \ldots \ldots \ldots \ldots \ldots \ldots$

4 Comparação de dois Modelos com Erros nas Variáveis 39

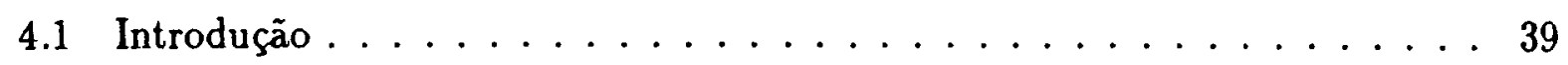

4.2 Fator de Atenuação Conhecido . . . . . . . . . . . 40

4.2 .1 Comentários Importantes: $\ldots \ldots \ldots \ldots \ldots$

4.3 Fator de Atenuação Desconhecido e Iguais . . . . . . . . . . 48

4.4 Ilustração Numérica $\ldots \ldots \ldots \ldots \ldots \ldots$

5 Teste da Razão de Verossimilhança $\quad 56$

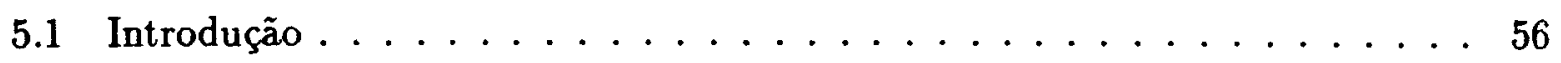

5.2 Teste da Razão de Verossimilança Generalizado . . . . . . . . . 57

5.3 Teste da Razão de Verossimilhança para Modelos Lineares . . . . . . . 58

5.4 Comparação do Teste da Razão de Verossimilhança com o Fator de Bayes a Posteriori . . . . . . . . . . . . . . . 63 


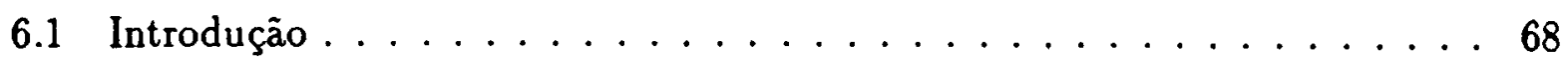

6.2 Formulação do Teste da Razão de Entropia a Posteriori . . . . . . . . . 69

6.3 Comparação de Modelos via Teste da Razão de Entropia a Posteriori . . 70

6.4 Comparação do Fator de Bayes a Posteriori com o Teste da Razão de Entropia a Posteriori . . . . . . . . . . . . . . 75

$\begin{array}{lll}7 & \text { Exemplo de Aplicação } & 87\end{array}$

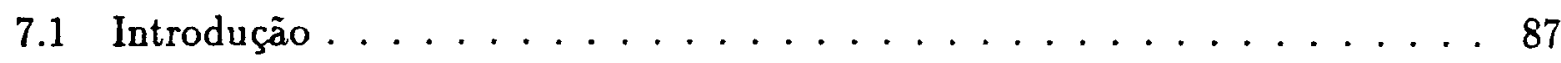

7.2 Obtenção dos Dados $\ldots \ldots \ldots \ldots \ldots \ldots \ldots$

7.3 Verificação da Adequação do Modelo . . . . . . . . . . . . . 89

7.4 Teste para Comparar os Coeficientes $\ldots \ldots \ldots \ldots \ldots \ldots$

8 Conclusões Finais e Perspectivas Futuras 98

8.1 Perspectivas Futuras $\ldots \ldots \ldots \ldots \ldots \ldots \ldots$

$\begin{array}{lll}\text { Apêndice 1 Fórmulas } & 101\end{array}$

Apêndice 2 Aproximação de Integrais pelo Método de Laplace 103

Apêndice 3 Família da Verossimilhança Penalizada 106 
Apêndice 4 Parâmetros Ortogonais

Apêndice 5 Tabelas das Constantes

Bibliografia 


\section{Capítulo 1}

\section{Introdução}

O problema de comparar modelos tem sido estudado com grande profundidade tanto do ponto de vista clássico como Bayesiano. Historicamente, a abordagem Bayesiana está baseada no fator de Bayes introduzido por Jeffreys em 1939. O fator de Bayes consiste em comparar modelos utilizando a razão das médias das funções de verossimilhança em relação as suas distribuições a priori, ou seja ,

$$
B=\frac{\bar{L}_{1}^{B}}{\bar{L}_{2}^{B}}
$$

onde

$$
\bar{L}_{j}^{B}=\int L_{j}\left(\Theta_{j}\right) \Pi_{j}\left(\Theta_{j}\right) d \Theta_{j} \quad ; j=1,2,
$$

é a média da função de verossimilhança $L_{j}$ em relação a priori $\Pi_{j}\left(\theta_{j}\right)$ e ao modelo $\mathbf{j}$.

O fator de Bayes proporciona, no enfoque Bayesiano, o peso de evidência do modelo $1\left(M_{1}\right)$ sobre o modelo $2\left(M_{2}\right)$. Este peso de evidência 
depende da priori $\Pi_{j}\left(\theta_{j}\right)$ e pode ser muito sensível as suas variações. Se a priori $\Pi_{j}\left(\Theta_{j}\right)$ representa uma opinião subjetiva e precisa acerca de $\Theta_{j}$, então tal sensibilidade não deve ser assunto de preocupação, mas muitos Bayesianos e não - Bayesianos sentem-se mais confortáveis com conclusões Bayesianas que são insensíveis a variações na priori do que com aquelas que são muito sensiveis (Box e Tiao, 1973).

Devido a ausência de um método preciso para formular a priori $\Pi_{j}\left(\Theta_{j}\right)$, que expressa a opinião subjetiva sobre $\theta_{j}$, o estatístico necessita de alguma maneira expressar a sua opinião sobre $\Theta_{j}$. O uso de difusa, vaga, ou priori de Jeffreys como representações de priori não informativa é muito comum em inferência Bayesiana, porém quando o espaço paramétrico é ilimitado e as hipóteses nulas são pontuais, isto pode levar ao paradoxo de Lindley (1957).

O paradoxo de Lindley ocorre quando o fator de Bayes aumenta com n (tamanho da amostra). Neste caso, para um n suficientemente grande teremos uma maior sustentação de $M_{1}$ na base do fator de Bayes, independente se $M_{1}$ é correto ou não. Podemos verificar este paradoxo através do seguinte exemplo citado em Aitkin (1991).

Seja $M_{1}$ um modelo normal com média $\mu_{1}$ e variância $\sigma^{2}$, com $\mu_{1}$ especificado, e $M_{2}$ um modelo normal com média $\mu_{2}$ e variância $\sigma^{2}$, com $\mu_{2}$ não especificado e $\sigma^{2}$ sendo conhecido. Considerando uma priori vaga sobre $\mu_{2}$ da forma $\Pi\left(\mu_{2}\right)=\frac{1}{2 C}$, onde $\mu_{2}$ pertence ao intervalo $(-C, C)$ e C é uma constante relativamente grande, o fator de Bayes para uma amostra de n observações é dado por: 


$$
B=2 C \frac{n^{1 / 2}}{\sigma} \phi(Z) /\left\{\Phi\left(n^{1 / 2} \frac{\bar{y}+C}{\sigma}\right)-\Phi\left(n^{1 / 2} \frac{\bar{y}-C}{\sigma}\right)\right\}
$$

onde o denominador da expressão acima rapidamente aproxima-se da unidade quando $\mathrm{C}$ aumenta. $\mathrm{O}$ valor de $\mathrm{B}$ pode ser arbitrariamente grande quando $\mathbf{C} \rightarrow \infty$ ou quando $\mathbf{n} \rightarrow \infty$, qualquer que seja o valor fixado de $Z$. Segundo Aitkin (1991), isto ocorre porque a priori uniforme coloca peso crescente para valores de $\mu_{2}$, quando $\mathrm{C}$ ou $\mathrm{n}$ tendem ao infinito, valores estes que são desprezíveis segundo a função de verossimilhança. Como a priori coloca pesos onde a função de verossimilhança é desprezível, podemos afirmar que a priori não tem que ser necessariamente uniforme, ou seja toda priori não uniforme mostrará que o fator de Bayes tenderá ao infinito quando $n$ cresce.

Estas dificuldades são bem conhecidas e têm sido freqüentemente discutidas; para maiores detalhes ver Shafer (1982). Este problema tem sido resolvido de diferentes formas. Para Casela e Berger (1987), o paradoxo não ocorre com modelos que especificam a hipótese através de intervalos e neste caso, as hipóteses nulas pontuais não são apropriadas, isto é, o paradoxo ocorre devido a forma da hipótese e não da análise do problema. Este argumento limita o uso da análise Bayesiana à hipóteses formuladas por intervalos e com isso excluindo a maioria dos problemas básicos de comparação de modelos.

Também tem sido argumentado que o paradoxo ocorre por causa do uso inadequado de priori não informativas. Na discussão de Shafer (1982), DeGroot diz, "Quando as distribuições a priori difusas são usadas em inferência Bayesiana, elas devem ser usadas com cuidado. Embora elas possam ser úteis em problemas de estimação, elas nunca são apropriadas para teste 
de hipótese".

Este argumento induz a utilização da análise Bayesiana com priori informativa, isto é, especificando a priori e avaliando a sua sensibilidade na conclusão dos resultados. Isto tem sido implementado por Smith e outros (1985).

Outras abordagens têm sido utilizadas com o objetivo de formular a distribuição a priori. Spiegelhalter e Smith (1982) propuseram um valor específico para $2 \mathrm{C}$ através de um mecanismo que eles chamaram de "Imaginary Training Sample" e aplicaram esta abordagem em casos genéricos.

Spiegelhalter e Smith (1980) também propuseram o uso da priori $\mathrm{N}\left(\mu_{1}, \sigma^{2} / n\right)$ para $\mu_{2}$, quando a alternativa em relação a hipótese nula é local. Neste caso, o fator de Bayes de $M_{1}$ para $M_{2}$ é dado por:

$$
B=2^{1 / 2} \exp \left\{-\frac{n\left(\bar{y}-\mu_{1}\right)^{2}}{4 \sigma^{2}}\right\}=2^{1 / 2} \exp \left\{-\frac{1}{4} Z^{2}\right\}
$$

Embora B em (1.1), não seja função de n e portanto não sofre do paradoxo de Lindley, exige um valor de $\mathrm{Z}$ muito grande para evidenciar contra $M_{1}$. Para um valor de $B$ de $1 / 20$ que corresponde a uma probabilidade a posteriori para $M_{1}$ de $1 / 21$, supondo uma priori não uniforme, requer $|Z|=3.66$.

Como a priori em (1.1) dá um peso maior para modelos $M_{2}$ numa vizinhança de $M_{1}$, Spiegelhalter e Smith denominaram este fator de Fator de Bayes Local. Apesar de ser apropriado em alguns problemas, é claro que 
quando $\mathrm{n}$ aumenta a priori sob $M_{2}$ favorece cada vez mais o modelo $M_{1}$, mesmo que o modelo alternativo $M_{2}$ seja apropriado. Esta abordagem, introduzida por Spiegelhalter e Smith, segundo Aitkin (1991) é questionável e deixa em aberto algumas questões relevantes.

Em virtude das desvantagens encontradas no fator de Bayes ou fator de Bayes local, Aitkin (1991) sugeriu um procedimento Bayesiano alternativo, que está baseado na média a posteriori em vez da média a priori, o qual denominou de Fator de Bayes a Posteriori. O fator de Bayes a posteriori, segundo o autor, teria algumas vantagens em relação ao fator de Bayes, por exemplo: é insensível em relação a priori e evita o paradoxo de Lindley.

O objetivo principal deste trabalho é utilizar o fator de Bayes a posteriori para comparar os coeficientes de regressão de modelos lineares e verificar as suas vantagens.

No Capítulo 2, definimos os modelos de regressão linear simples, sem erro de mensuração e com erro de mensuração, que serão usados para comparar os coeficientes de regressão, e também apresentamos formalmente o fator de Bayes a posteriori.

No Capítulo 3, comparamos dois modelos de regressão sem levar em conta o erro de mensuração nas variáveis. Para estes modelos consideramos três situações: a primeira considerando que as variâncias são iguais e desconhecidas, a segunda considerando que as variâncias são desiguais e desconhecidas e na terceira comparamos simultaneamente o coeficiente e a variância. Nos três casos verificamos, através de simulações, a sensibilidade em relação a priori e também o paradoxo de Lindley. 
No Capítulo 4, comparamos dois modelos de regressão levando em conta o erro de mensuração nas variáveis, onde a constante de atenuação, $K_{j}$, foi considerada conhecida e desconhecida. Também, através de simulaçōes, foram verificadas as vantagens do fator de Bayes a posteriori.

Embora o objetivo principal deste trabalho seja uma análise Bayesiana para comparar modelos, apresentamos no Capítulo 5 um teste para comparar modelos baseado no teste da razão de verossimilhança. Este teste foi incluído no trabalho, devido a necessidade de comparar o comportamento do fator de Bayes a posteriori com um procedimento não Bayesiano.

Também considerando o modelo de regressão linear sem erro de mensuração, propomos no Capítulo 6 um teste Bayesiano baseado no conceito de entropia introduzido por Shannon (1948), que denotamos de Teste da Razão de Entropia a Posteriori. Assim sendo, foi possivel fazer um estudo comparativo entre o fator de Bayes a posteriori encontrado no Capítulo 3 e este teste, utilizando para isso a escala de evidência de Jeffrey (1961).

No Capitulo 7, utilizando dados pertencentes a um trabalho de pesquisa de Vieira (1992), apresentamos um exemplo prático de aplicação do fator de Bayes a posteriori. Estes dados estão relacionados ao desenvolvimento de um sensor de corrente elétrica utilizando fibras ópticas.

Finalmente no Capítulo 8, são apresentadas algumas conclusões e comentários sobre as propostas futuras de pesquisa nesta área. 


\section{Capítulo 2}

\section{Formulação do Modelo e o Fator de Bayes a Posteriori}

\subsection{Introdução}

Neste capitulo definimos dois modelos que serão usados para comparar os coeficientes de regressão. O primeiro modelo a ser definido será sem erro nas variáveis que é um modelo de regressão linear simples passando pela origem e o segundo, é um modelo de regressão linear simples com erro de mensuração, onde faremos uma reparametrização ortogonal e chegaremos num modelo condicional.

Será introduzido o fator de Bayes a posteriori proposto por Aitkin (1991), que está baseado na média a posteriori da função de verossimilhança. O uso da média a posteriori tem algumas vantagens, que são: reduzir a sensibilidade para variações na priori e evitar o paradoxo de Lindley. 


\subsection{Variáveis sem Erro}

Toda metodologia de regressão linear envolve a especificação da relação linear entre variável resposta e variável preditora. Denotando a variável resposta ou variável dependente por $Y$ e $p$ variáveis preditoras ou variáveis independentes por $X_{1}, X_{2}, \ldots, X_{p}$, a relação linear tem a forma

$$
Y=\alpha+\beta_{1} X_{1}+\beta_{2} X_{2}+\ldots+\beta_{p} X_{p}+e,
$$

onde $\alpha, \beta_{1}, \beta_{2}, \ldots, \beta_{p}$ são parâmetros desconhecidos chamados de coeficientes de regressão. $O$ último termo, e, desta relação é inserido para refletir o fato que a resposta observada está sujeita a variabilidade e não pode ser expressa como uma combinação linear das variáveis preditoras. O erro, e , é devido a flutuação aleatória da resposta.

A regressão linear contendo somente uma variável independente é chamada de regressão linear simples. A regressão linear contendo mais que uma variável independente é referida como uma regressão linear múltipla.

Nesta seção vamos considerar dois modelos de regressão linear, passando pela origem $(\alpha=0)$, onde há somente uma variável independente.

Neste caso, o modelo é definido da seguinte maneira:

$$
\begin{aligned}
Y_{i j}=\beta_{j} X_{i j}+e_{i j} \quad, i & =1, \ldots, n_{j} \\
\mathbf{j} & =1,2,
\end{aligned}
$$


onde

$Y_{i j}$ : o valor da variável resposta ou variável dependente na í- ésima observação amostral para j - ésima população .

$\beta_{j}$ : parâmetro populacional.

$X_{i j}$ : variável conhecida, isto é, o valor da variável independente na i- ésima observação amostral para j - ésima população .

$e_{i j}$ : erro aleatório independente e normalmente distribuido com média zero e variância desconhecida $\sigma_{j}^{2}$, que denotamos por $e_{i j} \sim N\left(0, \sigma_{j}^{2}\right)$; $e_{i j}$ e $e_{r s}$ são não - correlacionados, i.e. $\operatorname{cov}\left(e_{i j}, e_{r s}\right)=0$ para todo $(\mathbf{i}, \mathbf{j}) \neq(\mathbf{r}, \mathbf{s})$

\subsection{Variáveis com Erros}

Apesar dos constantes avanços tecnológicos terem tornado cada vez mais precisos os procedimentos de mensuração, não é realista supor que tais variáveis são medidas sem erro e, o mais comum, é não termos acesso aos seus verdadeiros valores. Como o interesse recai, geralmente, em estimar os paramêtros de uma função, a presença de erros de mensuração afetará a precisão dos mesmos. Isto é importante em vários tipos de estudo, como por exemplo, no nosso caso onde queremos comparar a inclinação de duas regressões, onde parte da diferença encontrada (ou mesmo toda) pode ser devida a tais erros. 
Definiremos o modelo de regressão linear simples com erro de mensuração, onde $y_{i j}$ e $x_{i j}$ não são observados diretamente, ou seja, os valores observados são $Y_{i j}$ e $X_{i j}$, o modelo é dado por:

$$
\begin{aligned}
Y_{i j} & =y_{i j}+e_{i j} \\
X_{i j} & =x_{i j}+u_{i j} \\
y_{i j} & =\beta_{j} x_{i j}
\end{aligned}
$$

onde $i=1, \ldots, n_{j}$ e $j=1,2$. As variáveis aleatórias $x_{i j}, e_{i j}$ e $u_{i j}$ são independentes e normalmente distribuídas com média $\mu_{x}$, zero, zero e variâncias $\sigma_{x}^{2}, \sigma_{e}^{2}$, e $\sigma_{u}^{2}$, respectivamente, que denotamos por:

$$
\left(x_{i j}, e_{i j}, u_{i j}\right) \sim N \quad\left[\left(\mu_{x_{j}}, 0,0\right) ; \operatorname{diag}\left(\sigma_{x_{j}}^{2}, \sigma_{e_{j}}^{2}, \sigma_{u_{j}}^{2}\right)\right] .
$$

Consideramos também que $x_{i j}, e_{i j}$ e $u_{i j}$ são não correlacionados e $\mu_{x}$, conhecido. Sem perda de generalidade, vamos supor que $\mu_{x_{j}}=0$.

Usando resultados conhecidos da teoria de normal multivariada, verificamos que o par $\left(X_{i j}, Y_{i j}\right)$ tem uma distribuição normal bivariada com o vetor de médias e a matriz de covariância dados por:

$$
\left(\begin{array}{c}
X_{i j} \\
Y_{i j}
\end{array}\right) \sim N_{2}\left[\left(\begin{array}{c}
\mu_{x_{j}} \\
\beta_{j} \mu_{x_{j}}
\end{array}\right) ;\left(\begin{array}{cc}
\sigma_{x_{j}}^{2}+\sigma_{u j}^{2} & \beta_{j} \sigma_{x_{j}}^{2} \\
\beta_{j} \sigma_{x_{j}}^{2} & \beta_{j}^{2} \sigma_{x_{j}}^{2}+\sigma_{e_{j}}^{2}
\end{array}\right)\right]
$$

Da distribuição normal bivariada para $\left(X_{i j}, Y_{i j}\right)$ segue que a distribuição condicional (Rodrigues, Cordani - 1990 ) de $Y_{i j}$ dado $X_{i j}$ é normal 
com média $K_{j} X_{i j} \beta_{j}$ e variância $\sigma_{u,}^{2} K_{j} \beta_{j}^{2}+\sigma_{e j}^{2}$, onde

$$
K_{j}=\frac{\sigma_{x_{j}}^{2}}{\sigma_{x_{j}}^{2}+\sigma_{u_{j}}^{2}} .
$$

A constante $K_{j}$ é conhecida como fator de atenuação. Esta denomimação é decorrente do fato de $K_{j} \in[0,1]$. Outras denominações podem ser encontradas dependendo do contexto, por exemplo, coeficiente de atenuação, razão de confiabilidade, herdabilidade, comunalidade, etc. $O$ fator de atenuação $K_{j}$ é a proporção da variabilidade da quantidade não observável $\left(x_{j}\right)$ pela variabilidade da quantidade observável $\left(X_{j}\right)$ (ver Fuller, 1987).

Utilizando o procedimento dado por Cox \& Reid (1987) temos a seguinte reparametrização ortogonal (ver Apêndice 4) para o modelo condicional:

$$
\left\{\begin{array}{l}
\beta_{j}=\beta_{j} \\
\lambda_{j}=\sigma_{u_{j}}^{2} K_{j} \beta_{j}^{2}+\sigma_{e_{j}}^{2}
\end{array}\right.
$$

consequentemente,

$$
Y_{i j} / X_{i j} \sim N \quad\left[\begin{array}{lllll}
K_{j} & X_{i j} & \beta_{j} & , & \lambda_{j}
\end{array}\right]
$$




\subsection{Fator de Bayes a Posteriori. (Aitkin,1991)}

A comparação de modelos utilizando a razão das médias das funções de verossimilhança em relação as suas priori, ou fator de Bayes, foi introduzida por Jeffreys (1939) e intensamente utilizada por Good (1947) e Box e Hill (1967), dentre outros. Para uma referência mais completa sugerimos Spiegelhalter e Smith (1982). Uma teoria unificada dos vários critérios existentes para modelos lineares baseados no fator de Bayes, foi introduzida por Smith e Spiegelhalter (1980). Em um recente artigo, Aitkin (1991) discutiu as desvantagens do fator de Bayes e introduziu o fator de Bayes a posteriori como uma alternativa mais realista aos clássicos testes $\mathbf{F}$ para comparar modelos. Para calcular o fator de Bayes a posteriori, inicialmente temos que calcular a média a posteriori da função de verossimilhança $L_{j}\left(\Theta_{j}\right)$ dos dados observados segundo o modelo $M_{j}$, para $j=1,2$, isto é

$$
\bar{L}_{j}^{A}=\int L_{j}\left(\Theta_{j}\right) \Pi\left(\Theta_{j} / \text { dados }\right) d \Theta_{j},
$$

onde $\Pi\left(\Theta_{j} /\right.$ dados $)$ é a densidade a posteriori de $\Theta_{j}$ em relação a priori $\Pi_{j}\left(\Theta_{j}\right)$, isto é,

$$
\Pi\left(\Theta_{j} / \text { dados }\right)=\frac{L_{j}\left(\Theta_{j}\right) \Pi_{j}\left(\Theta_{j}\right)}{\int L_{j}\left(\Theta_{j}\right) \Pi_{j}\left(\Theta_{j}\right) d \Theta_{j}} .
$$

Logo, $\bar{L}_{j}^{A}$ pode ser expresso como 


$$
\begin{gathered}
\bar{L}_{j}^{A}=\frac{\int L_{j}^{2}\left(\Theta_{j}\right) \Pi_{j}\left(\Theta_{j}\right) d \Theta_{j}}{\int L_{j}\left(\Theta_{j}\right) \Pi_{j}\left(\Theta_{j}\right) d \Theta_{j}}, \text { ou }, \\
\bar{L}_{j}^{A}=\frac{l_{2}^{(j)}}{l_{1}^{(j)}},
\end{gathered}
$$

onde $l_{k}^{(j)}=\int L_{j}^{k}\left(\Theta_{j}\right) \Pi\left(\theta_{j}\right) d \Theta_{j}, j=1,2$.

O fator de Bayes a posteriori para o modelo $M_{1}$ versus o modelo $M_{2}$, é dado pela razão da média a posteriori denotado por $\mathbf{A}$ :

$$
A=\frac{\bar{L}_{1}^{A}}{\bar{L}_{2}^{A}} .
$$

O fator de Bayes a posteriori é uma medida da evidência da amostra a favor de $M_{1}$ contra o modelo $M_{2}$. Aitkin (1991) sugere que para valores de A menor que $\frac{1}{20}, \frac{1}{100}, \frac{1}{1000}$ constitui forte, muito forte e esmagadora evidência a favor de $M_{2}$ contra $M_{1}$. Para mais detalhes sobre o fator de Bayes a posteriori, sugerimos o artigo de Aitkin , 1991.

O fator de Bayes a posteriori possui algumas vantagens que são: reduzir a sensibilidade para variaçōes na priori e não ser afetado pelo paradoxo de Lindley. 


\section{Capítulo 3}

\section{Comparação de dois Modelos sem Erro nas Variáveis}

\subsection{Introdução}

O problema a ser resolvido consiste em utilizar o fator de Bayes a posteriori, introduzido no Capítulo 2, para comparar os coeficientes de dois modelos de regressão sem erros nas variáveis (2.1). Para isso usaremos densidades a priori não informativa e, como em geral os métodos Bayesianos estão sujeitos a algunas limitações relacionadas a resolução de integrais que podem não apresentar soluções analíticas explícitas, usaremos o método de Laplace para aproximação de integrais (ver por exemplo, Kass, Tierney e Kadane, 1990).

Vamos considerar em primeiro lugar o caso em que as variâncias são iguais e desconhecidas, $\sigma_{1}^{2}=\sigma_{2}^{2}=\sigma^{2}$ e em segundo, as variâncias desiguais e desconhecidas, $\sigma_{1}^{2} \neq \sigma_{2}^{2}$. O caso em que desejamos comparar simultaneamente os coeficientes e variâncias também será considerado. Finalizaremos 
com uma ilustração numérica realizada através de simulações de Monte Carlo.

\subsection{Comparação de dois Modelos de Regressão com Variâncias Iguais e Desconhecidas}

Nesta seção, consideramos o modelo definido em (2.1), porém com $e_{i j}$ sendo uma variável aleatória normalmente distribuída com média zero e variância desconhecida $\sigma^{2}$, que representamos por $e_{i j} \sim N\left(0, \sigma^{2}\right)$.

Nosso objetivo é testar a hipótese $H_{0}: \beta_{1}=\beta_{2}=\beta$ contra a hipótese alternativa $H_{1}: \beta_{1} \neq \beta_{2}$. O modelo sob $H_{0}$ chamaremos de $M_{1}$ e o modelo sob $H_{1}$ chamaremos de $M_{2}$.

Sob o modelo $M_{1}$, temos o parâmetro $\Theta_{1}=\left(\beta, \sigma^{2}\right)$ para o qual assumimos a priori não informativa da forma

$$
\Pi\left(\Theta_{1}\right) \propto \frac{1}{\left(\sigma^{2}\right)^{r}} .
$$

Para $r=\frac{3}{2},(3.1)$ é a priori de Jeffreys; para $r=1,(3.1)$ é a priori não informativa de Box Tiao (1974) e para $r=\frac{1}{2},(3.1)$ é uma priori difusa.

A função de verossimilhança é dada por:

$$
L_{1}\left(\Theta_{1}\right) \propto \frac{1}{\left(\sigma^{2}\right)^{\frac{n}{2}}} \exp \left\{-\frac{1}{2 \sigma^{2}} \sum_{j=1}^{2} \sum_{i=1}^{n_{j}}\left(Y_{i j}-\beta X_{i j}\right)^{2}\right\}
$$


Por outro lado, sobre o modelo $M_{2}$, temos o parâmetro $\Theta_{2}=\left(\beta_{1}, \beta_{2}, \sigma^{2}\right)$ para o qual consideramos a priori (3.1) e a função de verossimilhança como sendo:

$$
L_{2}\left(\Theta_{2}\right) \propto \frac{1}{\left(\sigma^{2}\right)^{\frac{n}{2}}} \quad \exp \left\{-\frac{1}{2 \sigma^{2}} \sum_{j=1}^{2} \sum_{i=1}^{n_{j}}\left(Y_{i j}-\beta_{j} X_{i j}\right)^{2}\right\},
$$

onde $n=n_{1}+n_{2}$.

\section{Lema 1}

Para o modelo definido em (2.1) com $\sigma_{1}^{2}=\sigma_{2}^{2}=\sigma^{2}$, sob $M_{1}$ temos que

$$
\bar{L}_{1}^{A}=\frac{\Gamma(n+r-1)}{\Gamma\left(\frac{n}{2}+r-1\right)}(R S S)^{-\frac{n}{2}} 2^{-\left(\frac{n}{2}+r-1\right)} 2^{-\frac{1}{2}} e^{-S / 2}
$$

onde RSS é dado por (3.7) e $S$ por (3.9).

\section{Prova:}

Combinando (3.1) e (3.2), obtemos:

$$
\begin{aligned}
l_{k}^{(1)} & \propto \int \frac{1}{\left(\sigma^{2}\right)^{\frac{k n}{2}+r}} \exp \left\{-\frac{k}{2 \sigma^{2}} \sum_{j=1}^{2} \sum_{i=1}^{n_{j}}\left(Y_{i j}-\beta X_{i j}\right)^{2}\right\} d \Theta_{1} \\
& =\iint \frac{1}{\left(\sigma^{2}\right)^{\frac{k n}{2}+r}} \exp \left\{-\frac{k}{2 \sigma^{2}} \sum_{j=1}^{2} \sum_{i=1}^{n_{j}}\left(Y_{i j}-\beta X_{i j}\right)^{2}\right\} d \sigma^{2} d \beta
\end{aligned}
$$


para $k=1,2$.

Integrando (3.5) em relação a $\sigma^{2}$ e usando a fórmula da integral dada no Apêndice 1.a, obtemos:

$$
l_{k}^{(1)} \propto \Gamma\left(\frac{k n}{2}+r-1\right) \int\left[\frac{k}{2} \sum_{j=1}^{2} \sum_{i=1}^{n j}\left(Y_{i j}-\beta X_{i j}\right)^{2}\right]^{-\left(\frac{k n}{2}+r-1\right)} d \beta .
$$

Se escrevermos a expressão acima em forma de exponencial e utilizarmos a identidade algébrica dada no Apêndice 1.b, obtemos:

$$
l_{k}^{(1)} \propto \Gamma\left(\frac{k n}{2}+r-1\right) \int \epsilon^{-\left(\frac{k n}{2}+r-1\right) \ln \left[\frac{k}{2}\left(R S S+\sum_{j=1}^{2} \sum_{i=1}^{n_{j}} X_{i j}^{2}\left(\beta-\widehat{\beta}_{j}\right)^{2}\right]\right.} d \beta
$$

onde

$$
\begin{aligned}
\hat{\beta}_{j} & =\frac{\sum_{i=1}^{n_{j}} Y_{i j} X_{i j}}{\sum_{i=1}^{n_{j}} X_{i j}^{2}} e \\
R S S & =\sum_{j=1}^{2} \sum_{i=1}^{n_{j}}\left(Y_{i j}-\hat{\beta}_{j} X_{i j}\right)^{2} .
\end{aligned}
$$

Tirando o termo que não depende de $\beta$ da integral, temos:

$l_{k}^{(1)} \propto \Gamma\left(\frac{k n}{2}+r-1\right)\left(\frac{k R S S}{2}\right)^{-\left(\frac{k n}{2}+r-1\right)} \int e^{-\left(\frac{k n}{2}+r-1\right) \ln \left(1+\frac{\sum_{j=1}^{2} \sum_{i=1}^{n} x_{i j}^{2}\left(\beta-\hat{\beta}_{j}\right)^{2}}{R S S}\right)} d \beta$.

Considerando a aproximação algébrica dada no Apêndice 1.c, a expressão acima pode ser escrita na forma: 
$l_{k}^{(1)} \propto \Gamma\left(\frac{k n}{2}+r-1\right)\left(\frac{k R S S}{2}\right)^{-\left(\frac{k n}{2}+r-1\right)} \int e^{-\left(\frac{k n}{2}+r-1\right)} \frac{\sum_{j=1}^{2} \sum_{i=1}^{n_{j}} x_{i j}^{2}\left(\theta-\hat{\theta}_{j}\right)^{2}}{k S S} d \beta$.

Observamos que a integral acima pode ser calculada, aproximadamente, utilizando o método de Laplace (ver por exemplo, Kass, Tierney e Kadane, 1990). Por isto, vamos escrever $l_{k}^{(1)}$ na forma:

$$
l_{k}^{(1)} \propto \Gamma\left(\frac{k n}{2}+r-1\right)\left(\frac{k R S S}{2}\right)^{-\left(\frac{k n}{2}+r-1\right)} \int f(\beta) \exp [-n h(\beta)] d \beta,
$$

onde

$$
\begin{aligned}
f(\beta) & =\exp \left\{-(r-1) \frac{\sum_{j=1}^{2} \sum_{i=1}^{n_{j}} X_{i j}^{2}\left(\beta-\hat{\beta}_{j}\right)^{2}}{R S S}\right\} e \\
-n h(\beta) & =-\frac{k n}{2} \frac{\sum_{j=1}^{2} \sum_{i=1}^{n_{j}} X_{i j}^{2}\left(\beta-\hat{\beta}_{j}\right)^{2}}{R S S} .
\end{aligned}
$$

Portanto, usando a aproximação de Laplace (ver Apêndice 2), temos:

$$
\begin{aligned}
l_{k}^{(1)} \propto & \Gamma\left(\frac{k n}{2}+r-1\right)\left(\frac{k R S S}{2}\right)^{-\left(\frac{k n}{2}+r-1\right)} \frac{\sqrt{2 \pi}}{\sqrt{k \sum_{j=1}^{2} a_{j}}} . \\
& . \exp \left\{-\frac{k S}{2}\right\} \exp \left\{-(r-1) \frac{\sum_{j=1}^{2} \sum_{i=1}^{n_{j}} X_{i j}^{2}\left(\hat{\beta}-\hat{\beta}_{j}\right)^{2}}{R S S}\right\},
\end{aligned}
$$

onde

$$
S=\sum_{j=1}^{2} a_{j}\left(\hat{\beta}-\hat{\beta}_{j}\right)^{2}
$$




$$
\begin{aligned}
\hat{\beta} & =\frac{\sum_{j=1}^{2} a_{j} \ddot{\beta}_{j}}{\sum_{j=1}^{2} a_{j}} e \\
a_{j} & =\frac{n \sum_{i=1}^{n} X_{i j}^{2}}{R S S} .
\end{aligned}
$$

Fazendo $\mathrm{k}=1,2 \mathrm{em}(3.8)$, temos respectivamente,

$$
\begin{aligned}
& l_{1}^{(1)} \propto \Gamma\left(\frac{n}{2}+r-1\right)\left(\frac{R S S}{2}\right)^{-\left(\frac{n}{2}+r-1\right)} \frac{\sqrt{2 \pi}}{\sqrt{\sum_{j=1}^{2} a_{j}}} . \\
& \quad \cdot \exp \left\{-\frac{S}{2}\right\} \exp \left\{-(r-1) \frac{\sum_{j=1}^{2} \sum_{i=1}^{n_{j}} X_{i j}^{2}\left(\hat{\beta}-\widehat{\beta}_{j}\right)^{2}}{R S S}\right\} e \\
& l_{2}^{(1)} \propto \Gamma(n+r-1)(R S S)^{-(n+r-1)} \frac{\sqrt{2 \pi}}{\sqrt{2 \sum_{j=1}^{2} a_{j}}} \cdot \\
& \quad \cdot \exp \{-S\} \exp \left\{-(r-1) \frac{\sum_{j=1}^{2} \sum_{i=1}^{n_{j}} X_{i j}^{2}\left(\hat{\beta}-\widehat{\beta}_{j}\right)^{2}}{R S S}\right\}
\end{aligned}
$$

Portanto, a média a posteriori é dada por:

$$
\bar{L}_{1}^{A}=\frac{l_{2}^{(1)}}{l_{1}^{(1)}}=\frac{\Gamma(n+r-1)}{\Gamma\left(\frac{n}{2}+r-1\right)}(R S S)^{-\frac{n}{2}} 2^{-\left(\frac{n}{2}+r-1\right)} 2^{-\frac{1}{2}} e^{-s / 2}
$$




\section{Lema 2}

Para o modelo definido em (2.1) com $\sigma_{1}^{2}=\sigma_{2}^{2}=\sigma^{2}$, sob $M_{2}$ temos que

$$
\bar{L}_{2}^{A}=\frac{\Gamma(n+r-1)}{\Gamma\left(\frac{n}{2}+r-1\right)}(R S S)^{-\frac{n}{2}} 2^{-\left(\frac{n}{2}+r-1\right)} 2^{-1}\left[\frac{n+r-1}{n+2 r-2}\right]^{-1},
$$

onde RSS é dado por (3.7).

\section{Prova:}

Combinando (3.1) e (3.3), obtemos:

$$
\begin{aligned}
l_{k}^{(2)} & \propto \int \frac{1}{\left(\sigma^{2}\right)^{\frac{k n}{2}+r}} \exp \left\{-\frac{k}{2 \sigma^{2}} \sum_{j=1}^{2} \sum_{i=1}^{n_{j}}\left(Y_{i j}-\beta_{j} X_{i j}\right)^{2}\right\} d \Theta_{2} \\
& =\iint \frac{1}{\left(\sigma^{2}\right)^{\frac{k n}{2}+r}} \exp \left\{-\frac{k}{2 \sigma^{2}} \sum_{j=1}^{2} \sum_{i=1}^{n_{j}}\left(Y_{i j}-\beta_{j} X_{i j}\right)^{2}\right\} d \sigma^{2} d \beta_{j}
\end{aligned}
$$

$\operatorname{para} k=1,2$.

Integrando (3.13) com respeito a $\sigma^{2}$, utilizando o mesmo procedimento usado em (3.5), obtemos:

$$
\begin{aligned}
l_{k}^{(2)} & \propto \Gamma\left(\frac{k n}{2}+r-1\right)\left(\frac{k R S S}{2}\right)^{-\left(\frac{k n}{2}+r-1\right)} \int e^{-\left(\frac{k n}{2}+r-1\right)} \frac{\sum_{j=1}^{2} \sum_{i=1}^{n_{j}} x_{i j}^{2}\left(\beta_{j}-\hat{\beta}_{j}\right)^{2}}{R S S} d \beta_{j}= \\
& =\Gamma\left(\frac{k n}{2}+r-1\right)\left(\frac{k R S S}{2}\right)^{-\left(\frac{k n}{2}+r-1\right)}\left[\prod_{j=1}^{2} \int e^{-\frac{1}{2}(k n+2 r-2) \frac{\sum_{i=1}^{n_{j}} x_{i j}^{2}}{R S S}\left(\beta_{j}-\hat{\beta}_{j}\right)^{2}} d \beta_{j}\right] .
\end{aligned}
$$


Integrando a expressão acima em relação a $\beta_{j}$, obtemos:

$$
l_{k}^{(2)} \propto \Gamma\left(\frac{k n}{2}+r-1\right)\left(\frac{k R S S}{2}\right)^{-\left(\frac{k n}{2}+r-1\right)}\left[\prod_{j=1}^{2} \sqrt{2 \pi}\left(\frac{R S S}{(k n+2 r-2) \sum_{i=1}^{n_{j}} X_{i j}^{2}}\right)^{\frac{1}{2}}\right]
$$

Fazendo $\mathrm{k}=1,2$, em (3.14), temos respectivamente,

$$
\begin{aligned}
& l_{1}^{(2)} \propto \Gamma\left(\frac{n}{2}+r-1\right)\left(\frac{R S S}{2}\right)^{-\left(\frac{n}{2}+r-1\right)}\left[\prod_{j=1}^{2} \sqrt{2 \pi}\left(\frac{R S S}{(n+2 r-2) \sum_{i=1}^{n_{j}} X_{i j}^{2}}\right)^{\frac{1}{2}}\right] e \\
& l_{2}^{(2)} \propto \Gamma(n+r-1)(R S S)^{-(n+r-1)}\left[\prod_{j=1}^{2} \sqrt{2 \pi}\left(\frac{R S S}{(2 n+2 r-2) \sum_{i=1}^{n_{j}} X_{i j}^{2}}\right)^{\frac{1}{2}}\right] .
\end{aligned}
$$

Portanto, a média a posteriori é dada por:

$$
\bar{L}_{2}^{A}=\frac{l_{2}^{(2)}}{l_{1}^{(2)}}=\frac{\Gamma(n+r-1)}{\Gamma\left(\frac{n}{2}+r-1\right)}(R S S)^{-\frac{n}{2}} 2^{-\left(\frac{n}{2}+r-1\right)} 2^{-1}\left[\frac{n+r-1}{n+2 r-2}\right]^{-1} \text {. }
$$

\section{Teorema 1}

O fator de Bayes a posteriori para o modelo (2.1) com $\sigma_{1}^{2}=\sigma_{2}^{2}=\sigma^{2}$ é dado por:

$$
A=\left[\frac{n+r-1}{n+2 r-2}\right] 2^{1 / 2} e^{-s / 2}
$$


onde $S$ é dado por (3.9).

Prova:

Da definição de fator de Bayes a posteriori que definimos no Capítulo 2 (2.6) temos que

$$
A=\frac{\bar{L}_{1}^{A}}{\bar{L}_{2}^{A}},
$$

onde $\bar{L}_{1}^{A}$ é dado por (3.4) e $\bar{L}_{2}^{A}$ é dado por (3.12). Logo substituindo em A (2.6) obtemos:

$$
A=\left[\frac{n+r-1}{n+2 r-2}\right] 2^{1 / 2} e^{-s / 2} .
$$

\subsubsection{Comentários Importantes:}

1. Sendo $-2 \ln A=S-\ln 2 C_{r}^{2}$ onde $C_{r}=\frac{n+r-1}{n+2 r-2}$, o fator de Bayes a posteriori em (3.15) pertence a família da verossimilhança penalizada proposta por Smith e Spiegelhalter (1980) para $\nu=1$ e $m=\ln 2 C_{r}^{2}$ (ver Apêndice 3). É importante observar que para $r=1$, temos que $C_{r}=1$ logo $m=\ln 2$ o qual coincide com o resultado da Seção 3 de Aitkin (1991).

2. Verificamos que $a_{j}$ dado por (3.11) pode ser colocado na forma $a_{j}=\frac{\sum_{i=1}^{n_{j}} X_{i j}^{2}}{R S S / n}$ onde $\frac{R S S}{n}$ seria a estimativa da variância comum $\sigma^{2}$, 
ou seja, $\hat{\sigma}^{2}=\frac{R S S}{n}$. Logo podemos escrever o coeficiente $a_{j}$ como $a_{j}=\frac{1}{\hat{\sigma}^{2} / \sum_{i=1}^{n} x_{j,}^{2}}=\frac{1}{\sqrt{V_{a r}\left(\hat{\beta}_{j}\right)}}$. Portanto $a_{j}$ é o inverso da variância estimada de $\hat{\beta}_{j}$ ou a medida de informação de Fisher estimada.

3. Em (3.6) $\widehat{\beta}_{j}$ é o estimador de $\beta_{j}$ e em (3.10) $\widehat{\beta}$ é a média dos estimadores $\hat{\beta}_{j}$ ponderado pelo $a_{j}$ citado no item anterior. Logo, a população com mais informação terá maior peso em $\hat{\beta}$.

4. A estatística $S$ (3.9), ponderado por $a_{j}$, compara o coeficiente estimado de cada população $\left(\hat{\beta}_{j}\right)$ com o estimador comum de beta $(\hat{\beta})$.

\subsection{Comparação de dois Modelos de Regressão com Variâncias Desiguais e Desconhecidas}

Nesta seção, consideramos o modelo definido em (2.1) com $\sigma_{1}^{2} \neq$ $\sigma_{2}^{2}$, ou seja,

$$
\begin{aligned}
& Y_{i j}=\beta_{j} X_{i j}+e_{i j} \\
& e_{i j} \sim N\left(0, \sigma_{j}^{2}\right),
\end{aligned}
$$

onde $i=1,2, \ldots, n_{j}$ e $j=1,2$. 
Seja $M_{1}$ o modelo correspondente a $H_{0}: \beta_{1}=\beta_{2}=\beta \mathrm{e}$ $\Theta_{1}=\left(\beta, \sigma_{1}^{2}, \sigma_{2}^{2}\right)$. Supondo independência entre as populações, usamos a seguinte priori:

$$
\Pi\left(\Theta_{1}\right) \propto \frac{1}{\left(\sigma_{1}^{2}\right)^{r}\left(\sigma_{2}^{2}\right)^{r}} .
$$

A função de verossimilhança é dada por:

$$
L_{1}\left(\Theta_{1}\right) \propto \frac{1}{\left(\sigma_{1}^{2}\right)^{\frac{n_{1}}{2}}\left(\sigma_{2}^{2}\right)^{\frac{n_{2}}{2}}} \exp \left\{-\frac{1}{2} \sum_{j=1}^{2} \frac{1}{\sigma_{j}^{2}} \sum_{i=1}^{n_{j}}\left(Y_{i j}-\beta X_{i j}\right)^{2}\right\} .
$$

Sob o modelo $M_{2}$ correspondente a $H_{1}: \beta_{1} \neq \beta_{2}$, temos o parâmetro $\Theta_{2}=\left(\beta_{1}, \beta_{2}, \sigma_{1}^{2}, \sigma_{2}^{2}\right)$. Assumimos a priori (3.16) para $\Theta_{2}$, e a função de verossimilhança é dada por:

$$
L_{2}\left(\Theta_{2}\right) \propto \frac{1}{\left(\sigma_{1}^{2}\right)^{\frac{n_{1}}{2}}\left(\sigma_{2}^{2}\right)^{\frac{n_{2}}{2}}} \exp \left\{-\frac{1}{2} \sum_{j=1}^{2} \frac{1}{\sigma_{j}^{2}} \sum_{i=1}^{n_{j}}\left(Y_{i j}-\beta_{j} X_{i j}\right)^{2}\right\} .
$$

\section{Lema 3}

Para o modelo definido em (2.1) com $\sigma_{1}^{2} \neq \sigma_{2}^{2}$, sob $M_{1}$ temos que

$$
\bar{L}_{1}^{A}=\left[\prod_{j=1}^{2} \frac{\Gamma\left(n_{j}+r-1\right)}{\Gamma\left(\frac{n_{j}}{2}+r-1\right)}\left(R S S_{j}\right)^{-\frac{n_{j}}{2}} 2^{-\left(\frac{n_{j}}{2}+r-1\right)}\right] 2^{-\frac{1}{2}} e^{-S / 2}
$$

onde $R S S_{j}$ é dado por (3.23) e $S$ é dado por (3.25). 


\section{Prova:}

Combinando (3.16) e (3.17), obtemos:

$$
\begin{aligned}
l_{k}^{(1)} & \propto \int \frac{1}{\left(\sigma_{1}^{2}\right)^{\frac{k n_{1}}{2}+r}\left(\sigma_{2}^{2}\right)^{\frac{k n_{2}}{2}+r}} \exp \left\{-\frac{k}{2} \sum_{j=1}^{2} \frac{1}{\sigma_{j}^{2}} \sum_{i=1}^{n_{j}}\left(Y_{i j}-\beta X_{i j}\right)^{2}\right\} d \Theta_{1} \\
& =\int\left[\prod_{j=1}^{2} \int \frac{1}{\left(\sigma_{j}^{2}\right)^{\frac{k n_{j}}{2}+r}} \exp \left\{-\frac{k}{2 \sigma_{j}^{2}} \sum_{i=1}^{n_{j}}\left(Y_{i j}-\beta X_{i j}\right)^{2}\right\} d \sigma_{j}^{2}\right] d \beta
\end{aligned}
$$

para $k=1,2$.

Integrando (3.20) em relação a $\sigma_{j}^{2}$, usando a fórmula da integral dada no Apêndice 1.a, obtemos:

$$
l_{k}^{(1)} \propto \int \prod_{j=1}^{2}\left[\frac{k}{2} \sum_{i=1}^{n_{j}}\left(Y_{i j}-\beta X_{i j}\right)^{2}\right]^{-\left(\frac{k n_{j}}{2}+r-1\right)} \Gamma\left(\frac{k n_{j}}{2}+r-1\right) d \beta .
$$

Escrevendo (3.21) em forma exponencial e utilizando a identidade algébrica dada no Apêndice 1.b, obtemos:

$$
\begin{aligned}
l_{k}^{1} \propto & {\left[\prod_{j=1}^{2} \Gamma\left(\frac{k n_{j}}{2}+r-1\right)\left(\frac{k R S S_{j}}{2}\right)^{-\left(\frac{k n_{j}}{2}+r-1\right)}\right] } \\
& \cdot \int \exp \left\{-\sum_{j=1}^{2}\left(\frac{k n_{j}}{2}+r-1\right) \ln \left(1+\frac{\sum_{i=1}^{n_{j}} X_{i j}^{2}\left(\beta-\hat{\beta}_{j}\right)^{2}}{R S S_{j}}\right)\right\} d \beta,
\end{aligned}
$$


onde

$$
\begin{aligned}
\hat{\beta}_{j} & =\frac{\sum_{i=1}^{n,} Y_{i j} X_{i j}}{\sum_{i=1}^{n,} X_{i j}^{2}} e \\
R S S_{j} & =\sum_{i=1}^{n,}\left(Y_{i j}-\hat{\beta}_{j} X_{i j}\right)^{2} .
\end{aligned}
$$

Considerando a aproximação algébrica dada no Apêndice 1.c na expressão acima, temos:

$l_{k}^{(1)} \propto\left[\prod_{j=1}^{2} \Gamma\left(\frac{k n_{j}}{2}+r-1\right)\left(\frac{k R S S_{j}}{2}\right)^{-\left(\frac{k n_{j}}{2}+r-1\right)}\right] \int e^{-\sum_{j=1}^{2}\left(\frac{k n_{j}}{2}+r-1\right) \frac{\sum_{i=1}^{n_{j}, x_{i j}^{2}\left(\beta-\widehat{\beta}_{j}\right)^{2}}}{R S S_{j}}} d \beta$.

Como não conseguimos achar uma solução analítica explícita para a integral acima, exploramos o uso do método de Laplace (ver por exemplo, Kass, Tierney e Kadane, 1990). Escrevendo na forma:

$$
l_{k}^{(1)} \propto\left[\prod_{j=1}^{2} \Gamma\left(\frac{k n_{j}}{2}+r-1\right)\left(\frac{k R S S_{j}}{2}\right)^{-\left(\frac{k n_{2}}{2}+r-1\right)}\right] \int f(\beta) \exp [-n h(\beta)] d \beta
$$

onde

$$
\begin{aligned}
f(\beta) & =\exp \left\{-\sum_{j=1}^{2}(r-1) \frac{\sum_{i=1}^{n_{j}} X_{i j}^{2}\left(\beta-\hat{\beta}_{j}\right)^{2}}{R S S_{j}}\right\} e \\
-n h(\beta) & =-\frac{k}{2} \sum_{j=1}^{2} \frac{n_{j} \sum_{i=1}^{n_{j}} X_{i j}^{2}\left(\beta-\hat{\beta}_{j}\right)^{2}}{R S S_{j}}
\end{aligned}
$$


Portanto, usando a aproximação de Laplace (ver Apêndice 2), temos:

$$
\begin{aligned}
l_{k}^{(1)} \propto & {\left[\prod_{j=1}^{2} \Gamma\left(\frac{k n_{j}}{2}+r-1\right)\left(\frac{k R S S_{j}}{2}\right)^{-\left(\frac{k n_{j}}{2}+r-1\right)}\right] \frac{\sqrt{2 \pi}}{\sqrt{k \sum_{j=1}^{2} a_{j}}} . } \\
& . \exp \left\{-\frac{k S}{2}\right\} \exp \left\{-\sum_{j=1}^{2}(r-1) \frac{\sum_{i=1}^{n_{j}} X_{i j}^{2}\left(\hat{\beta}-\widehat{\beta}_{j}\right)^{2}}{R S S_{j}}\right\}
\end{aligned}
$$

onde

$$
\begin{aligned}
& S=\sum_{j=1}^{2} a_{j}\left(\hat{\beta}-\hat{\beta}_{j}\right)^{2}, \\
& \hat{\beta}=\frac{\sum_{j=1}^{2} a_{j} \hat{\beta}_{j}}{\sum_{j=1}^{2} a_{j}} e \\
& a_{j}=\frac{n_{j} \sum_{i=1}^{n} X_{i j}^{2}}{R S S_{j}} .
\end{aligned}
$$

Fazendo $k=1,2 \mathrm{em}(3.24)$, temos respectivamente ,

$$
\begin{aligned}
l_{1}^{(1)} \propto & {\left[\prod_{j=1}^{2} \Gamma\left(\frac{n_{j}}{2}+r-1\right)\left(\frac{R S S_{j}}{2}\right)^{-\left(\frac{n_{j}}{2}+r-1\right)}\right] \frac{\sqrt{2 \pi}}{\sqrt{\sum_{j=1}^{2} a_{j}}} . } \\
& . \exp \left\{-\frac{S}{2}\right\} \exp \left\{-\sum_{j=1}^{2}(r-1) \frac{\sum_{i=1}^{n_{j}} X_{i j}^{2}\left(\hat{\beta}-\hat{\beta}_{j}\right)^{2}}{R S S_{j}}\right\} e
\end{aligned}
$$




$$
\begin{aligned}
l_{2}^{(1)} \propto & {\left[\prod_{j=1}^{2} \Gamma\left(n_{j}+r-1\right)\left(R S S_{j}\right)^{-\left(n_{j}+r-1\right)}\right] \frac{\sqrt{2 \pi}}{\sqrt{2 \sum_{j=1}^{2} a_{j}}} . } \\
& \cdot \exp \{-S\} \quad \exp \left\{-\sum_{j=1}^{2}(r-1) \frac{\sum_{i=1}^{n} X_{i j}^{2}\left(\hat{\beta}-\hat{\beta}_{j}\right)^{2}}{R S S_{j}}\right\} .
\end{aligned}
$$

Portanto, a média a posteriori é dada por:

$$
\bar{L}_{1}^{A}=\frac{l_{2}^{(1)}}{l_{1}^{(1)}}=\left[\prod_{j=1}^{2} \frac{\Gamma\left(n_{j}+r-1\right)}{\Gamma\left(\frac{n_{j}}{2}+r-1\right)}\left(R S S_{j}\right)^{-\frac{n_{j}}{2}} 2^{-\left(\frac{n_{j}}{2}+r-1\right)}\right] 2^{-\frac{1}{2}} e^{-S / 2} .
$$

\section{Lema 4}

Para o modelo definido em (2.1) com $\sigma_{1}^{2} \neq \sigma_{2}^{2}$, sob $M_{2}$ temos que

$$
\bar{L}_{2}^{A}=\prod_{j=1}^{2} \frac{\Gamma\left(n_{j}+r-1\right)}{\Gamma\left(\frac{n_{j}}{2}+r-1\right)}\left(R S S_{j}\right)^{-\frac{n_{j}}{2}} 2^{-\left(\frac{n_{j}}{2}+r-1\right)} 2^{-\frac{1}{2}}\left(\frac{n_{j}+r-1}{n_{j}+2 r-2}\right)^{-\frac{1}{2}},
$$

onde $R S S_{j}$ é dado por (3.23).

\section{Prova:}

Combinando (3.16) e (3.18), obtemos: 


$$
\begin{aligned}
l_{k}^{(2)} & \propto \int \frac{1}{\left(\sigma_{1}^{2}\right)^{\frac{k n_{1}}{2}+r}\left(\sigma_{2}^{2}\right)^{\frac{k n_{2}}{2}+r}} \exp \left\{-\frac{k}{2} \sum_{j=1}^{2} \frac{1}{\sigma_{j}^{2}} \sum_{i=1}^{n_{1}}\left(Y_{i j}-\beta_{j} X_{i j}\right)^{2}\right\} d \Theta_{2} \\
& =\int\left[\prod_{j=1}^{2} \int \frac{1}{\left(\sigma_{j}^{2}\right)^{\frac{k n_{1}}{2}+r}} \exp \left\{-\frac{k}{2 \sigma_{j}^{2}} \sum_{i=1}^{n_{j}}\left(Y_{i j}-\beta_{j} X_{i j}\right)^{2}\right\} d \sigma_{j}^{2}\right] d \beta_{j}
\end{aligned}
$$

para $k=1,2$.

Integrando (3.29) com respeito a $\sigma_{j}^{2}$ utilizando o mesmo procedimento usado em (3.20), obtemos:

$$
l_{k}^{(2)} \propto \prod_{j=1}^{2} \Gamma\left(\frac{k n_{j}}{2}+r-1\right)\left(\frac{k R S S_{j}}{2}\right)^{-\left(\frac{k n_{j}}{2}+r-1\right)} \int e^{-\left(\frac{k n_{j}}{2}+r-1\right) \frac{\sum_{i=1}^{n_{j} x_{i j}^{2}\left(\beta_{j}-\hat{\beta}_{j}\right)^{2}}}{R S S_{j}}} d \beta_{j}
$$

Integrando a expressão acima em relação a $\beta_{j}$ e aproximando a normal, obtemos:

$$
l_{k}^{(2)} \propto \prod_{j=1}^{2} \Gamma\left(\frac{k n_{j}}{2}+r-1\right)\left(\frac{k R S S_{j}}{2}\right)^{-\left(\frac{k n_{j}}{2}+r-1\right)} \sqrt{2 \pi}\left(\frac{R S S_{j}}{\left(k n_{j}+2 r-2\right) \sum_{i=1}^{n_{j}} X_{i j}^{2}}\right)^{\frac{1}{2}}
$$

Fazendo $\mathrm{k}=1,2 \mathrm{em}(\mathbf{3 . 3 0})$, temos respectivamente,

$$
l_{1}^{(2)} \propto \prod_{j=1}^{2} \Gamma\left(\frac{n_{j}}{2}+r-1\right)\left(\frac{R S S_{j}}{2}\right)^{-\left(\frac{n_{j}}{2}+r-1\right)} \sqrt{2 \pi}\left(\frac{R S S_{j}}{\left(n_{j}+2 r-2\right) \sum_{i=1}^{n_{j}} X_{i j}^{2}}\right)^{\frac{1}{2}} e
$$


$l_{2}^{(2)} \propto \prod_{j=1}^{2} \Gamma\left(n_{j}+r-1\right)\left(R S S_{j}\right)^{-(n,+r-1)} \sqrt{2 \pi}\left(\frac{R S S_{j}}{\left(2 n_{j}+2 r-2\right) \sum_{i=1}^{n_{j}} X_{i j}^{2}}\right)^{\frac{1}{2}}$.

Portanto, a média a posteriori é dada por:

$\bar{L}_{2}^{A}=\frac{l_{2}^{(2)}}{l_{1}^{(2)}}=\prod_{j=1}^{2} \frac{\Gamma\left(n_{j}+r-1\right)}{\Gamma\left(\frac{n_{1}}{2}+r-1\right)}\left(R S S_{j}\right)^{-\frac{n_{j}}{2}} 2^{-\left(\frac{n_{j}}{2}+r-1\right)} 2^{-\frac{1}{2}}\left(\frac{n_{j}+r-1}{n_{j}+2 r-2}\right)^{-\frac{1}{2}}$.

Teorema 2

O fator de Bayes a posteriori para o modelo (2.1) com $\sigma_{1}^{2} \neq \sigma_{2}^{2}$ é dado por:

$$
A=\left[\prod_{j=1}^{2} \frac{n_{j}+r-1}{n_{j}+2 r-2}\right]^{\frac{1}{2}} 2^{1 / 2} e^{-s / 2}
$$

onde $S$ é dado por (3.25).

Prova:

Segue basicamente do Lema 3 e Lema 4 e da definição de fator de Bayes a posteriori.

\subsubsection{Comentários Importantes:}

1. Sendo $-2 \ln A=S-\ln 2 C_{r}$ onde $C_{r}=\left[\Pi_{j=1}^{2} \frac{n_{j}+r-1}{n_{j}+2 r-2}\right]$, novamente, $-2 \ln A$ pertence a família da verossimilhança penalizada 
proposta por Smith e Spiegelhalter (1980) para $\nu=1$ e $m=\ln 2 C_{r}$ (ver Apêndice 3). Quando $r=1$, temos que $C_{r}=1$ e $m=\ln 2$, o qual coincide com o resultado da Seção 3 de Aitkin (1991).

2. A expressão (3.27) pode ser escrita da forma $a_{j}=\frac{\sum_{i=1}^{n_{j}} X_{i, j}^{2}}{R S S_{j} / n_{j}}$ onde $\frac{R S S_{j}}{n_{j}}$ seria a estimativa da variância de cada população, ou seja $\hat{\sigma}_{j}^{2}=\frac{R S S_{1}}{n_{j}}$. Logo podemos escrever $a_{j}=\frac{1}{\hat{\sigma}_{j}^{2} / \sum_{i=1}^{n} X_{i j}^{2}}=\frac{1}{\overline{V a r}\left(\hat{\beta}_{j}\right)}$, portanto $a_{j}$ é o inverso da variância estimada de $\widehat{\beta}_{j}$ ou a medida de informação de Fisher estimada. Na seção anterior, o $a_{j}$ estava relacionado à variância comum as populações $\sigma^{2}$, enquanto aqui está relacionado à variância de cada população $\sigma_{j}^{2}$ para $j=1,2$.

3. Em (3.22) $\widehat{\beta}_{j}$ é o estimador de $\beta_{j}$ e em (3.26) $\hat{\beta}$ é a média dos estimadores $\beta_{j}$ ponderado pelo $a_{j}$ citado no item anterior. Logo a população com mais informação terá maior peso em $\hat{\beta}$.

\subsection{Comparação Simultânea dos Coeficientes e Va- riâncias}

O objetivo desta seção é comparar simultaneamente os coeficientes e as variâncias de dois modelos de regressão, isto é, vamos testar a hipótese $H_{0}: \beta_{1}=\beta_{2}=\beta$ e $\sigma_{1}^{2}=\sigma_{2}^{2}=\sigma^{2}$ ( modelo $M_{1}$ ) contra a hipótese alternativa $H_{1}: \beta_{1} \neq \beta_{2}$ e $\sigma_{1}^{2} \neq \sigma_{2}^{2}$ ( modelo $M_{2}$ ). Consideramos para isso o modelo definido em (2.1) que é: 


$$
\begin{aligned}
& Y_{i j}=\beta_{j} X_{i j}+e_{i j} \\
& e_{i j} \sim N\left(0, \sigma_{j}^{2}\right),
\end{aligned}
$$

onde $i=1,2, \ldots, n_{j} e j=1,2$.

Sob o modelo $M_{1}$, temos o parâmetro $\Theta_{1}=\left(\beta, \sigma^{2}\right)$ que coincide com o parâmetro do modelo $M_{1}$ definido na Seção (3.2) . Logo assumindo a priori (3.1) e a função de verossimilhança (3.2), temos como média a posteriori o resultado do Lema 1, isto é,

$$
\bar{L}_{1}^{A}=\frac{\Gamma(n+r-1)}{\Gamma\left(\frac{n}{2}+r-1\right)}(R S S)^{-\frac{n}{2}} 2^{-\left(\frac{n}{2}+r-1\right)} 2^{-\frac{1}{2}} e^{-S / 2} .
$$

Sob o modelo $M_{2}$, temos o parâmetro $\Theta_{2}=\left(\beta_{1}, \beta_{2}, \sigma_{1}^{2}, \sigma_{2}^{2}\right)$ que coincide com o parâmetro do modelo $M_{2}$ da Seção (3.3). Então a priori é (3.16) e a função de verossimilhança é (3.18). Assim sendo, temos como média a posteriori o resultado do Lema 4 que é:

$$
\bar{L}_{2}^{A}=\prod_{j=1}^{2} \frac{\Gamma\left(n_{j}+r-1\right)}{\Gamma\left(\frac{n_{j}}{2}+r-1\right)}\left(R S S_{j}\right)^{-\frac{n_{j}}{2}} 2^{-\left(\frac{n_{j}}{2}+r-1\right)} 2^{-\frac{1}{2}}\left(\frac{n_{j}+r-1}{n_{j}+2 r-2}\right)^{-\frac{1}{2}}
$$


Teorema 3

O fator de Bayes a posteriori para comparar simultaneamente coeficiente e variância é dado por:

$$
A=\frac{(R S S)^{-\frac{n}{2}}}{\prod_{j=1}^{2}\left(R S S_{j}\right)^{-\frac{n_{1}}{2}}} C_{\tau} 2^{\frac{1}{2}} e^{-S / 2}
$$

onde $C_{\tau}$ é constante denominada por

$$
C_{\tau}=\left[\frac{\frac{\Gamma(n+r-1)}{\Gamma\left(\frac{n}{2}+r-1\right)}}{\prod_{j=1}^{2} \frac{\Gamma\left(n_{j}+r-1\right)}{\Gamma\left(\frac{n_{2}}{2}+r-1\right)}}\right]\left[\prod_{j=1}^{2}\left(\frac{n_{j}+r-1}{n_{j}+2 r-2}\right)^{\frac{1}{2}}\right] 2^{(r-1)},
$$

$R S S$ por (3.7), $R S S_{j}$ por (3.23) e $S$ por (3.9).

Prova:

Segue basicamente do Lema 1 e Lema 4 e da definição de fator de Bayes a posteriori.

\subsubsection{Interpretações}

1. Sendo $-2 \ln A=n \ln R S S-\ln \left(\prod_{j=1}^{2}\left(R S S_{j}\right)^{n_{j}}\right)+S-\ln 2 C_{r}^{2}$, notamos que $-2 \ln A$ não pertence a familia da verossimilhança penalizada proposta por Smith e Spiegelhalter (1980). 
2. Notamos o aparecimento do termo $\frac{(R S S)^{-}-\frac{\pi}{2}}{\prod_{j=1}^{2}\left(R S S_{j}\right)^{-\frac{\pi}{2}}}$ em (3.32) que é a contribuição da variância no fator de Bayes a posteriori, já que estamos também interessados en compará-las.

\subsection{Ilustração Numérica}

Nesta seção apresentamos uma ilustração numérica com a finalidade de verificar o comportamento do fator de Bayes a posteriori obtido em (3.15), (3.31) e (3.32). Usamos para isso a simulação de Monte Carlo, realizada em um micro - computador PC/AT com o auxílio do programa Minitab, para gerar as distribuições normais.

Procedemos de maneira análoga para os três casos, isto é, foram geradas 50 amostras de acordo com o modelo especificado em cada seção com $n_{1}=n_{2}=10,30$ e $50 ; \beta_{1}=\beta_{2}=1$; $X_{i 1} \sim N(30,2) \quad e \quad X_{i 2} \sim N(40,2)$. Para o caso da seção (3.2) e (3.4) onde as variâncias são iguais, consideramos $\sigma_{1}^{2}=\sigma_{2}^{2}=1$, enquanto que para a seção (3.3) em que as variâncias são diferentes, consideramos $\sigma_{1}^{2}=1$ e $\sigma_{2}^{2}=4$. Para cada amostra gerada foi calculado o fator de Bayes a posteriori. Repetimos este procedimento 50 vezes e achamos a média do fator de Bayes a posteriori, ou seja $\frac{\sum_{i=1}^{50} A_{i}}{50}$, o qual mostramos a seguir na Tabela 3.1 . 


\begin{tabular}{|c|c|c|c|c|}
\hline \multirow[b]{2}{*}{ F.B.P. } & \multirow[b]{2}{*}{$n_{1}=n_{2}$} & \multicolumn{3}{|c|}{$\mathbf{r}$} \\
\hline & & $1 / 2$ & 1 & $3 / 2$ \\
\hline (3.15) & $\begin{array}{l}10 \\
30 \\
50\end{array}$ & $\begin{array}{l}1.03243 \\
0.9944 \\
1.03496\end{array}$ & $\begin{array}{l}1.00595 \\
0.98604 \\
1.03236\end{array}$ & $\begin{array}{l}0.99391 \\
0.97795 \\
1.02725\end{array}$ \\
\hline$(3.31)$ & $\begin{array}{l}10 \\
30 \\
50\end{array}$ & $\begin{array}{l}1.12606 \\
1.01775 \\
0.97998\end{array}$ & $\begin{array}{l}1.06679 \\
1.00051 \\
0.970085\end{array}$ & $\begin{array}{c}1.0183 \\
0.98437 \\
0.96058\end{array}$ \\
\hline (3.32) & $\begin{array}{l}10 \\
30 \\
50\end{array}$ & $\begin{array}{l}1.06917 \\
0.82497 \\
0.80802\end{array}$ & $\begin{array}{l}0.9506 \\
0.79532 \\
0.79071\end{array}$ & $\begin{array}{l}0.89059 \\
0.77761 \\
0.78001\end{array}$ \\
\hline
\end{tabular}

Tabela 3.1: Valor médio de A para dados gerados segundo $M_{1}$ 
Nota-se da Tabela 3.1 que, em média, o fator de Bayes a posteriori detecta a igualdade dos $\beta$, ou seja, aceita com bastante ênfase o modelo $M_{1}$. Os valores obtidos são próximos de 1 , ou melhor, acima de 0.05 que seria o valor que rejeitaria o modelo $M_{1}$ (Capítulo 2 ).

Verificamos também que o fator de Bayes a posteriori é insensivel em média com relação a priori haja vista que ele detecta a igualdade $\operatorname{dos} \beta, \operatorname{com}$ bastante convicção, independente do tipo de priori que estamos utilizando. A Tabela 3.1 também mostra numericamente que o fator de Bayes a posteriori não apresenta o paradoxo de Lindley, pois é relativamente estável em relação a n.

Apesar dos resultados obtidos serem satisfatórios, resolvemos fazer uma outra simulação, agora considerando a hipótese alternativa (Modelo $M_{2}$ ) e o fator de Bayes a posteriori obtido em (3.32), isto porque queríamos nos certificar de que realmente o fator de Bayes a posteriori não sofria do paradoxo de Lindley, pois ao calcularmos a constante $C_{r}$, verificamos que ela não se comportava como nos casos anteriores, ou seja, o seu valor aumentava com o tamanho da amostra, como podemos ver na Tabela 3.2 , enquanto que para os dois casos anteriores os $C_{r}$ eram praticamentes iguais em todas as amostras (ver Apèndice 5).

O procedimento para esta simulação foi o mesmo usado anteriormente, só que agora como está baseado no modelo $M_{2}$, onde os betas e as variâncias são diferentes, consideramos $\beta_{1}=1 e \beta_{2}=3 ; \sigma_{1}^{2}=1 e$ $\sigma_{2}^{2}=4$. Na Tabela 3.3 apresentamos o valor médio do fator de Bayes a posteriori (3.32). 


\begin{tabular}{|c|ccc|}
\hline \multirow{2}{*}{$n_{1}=n_{2}$} & $1 / 2$ & \multicolumn{4}{|c|}{$C_{\text {厂 }}$} \\
\cline { 2 - 4 } 10 & 1649.2099 & 1466.3175 & $13 / 2$ \\
\hline \hline 30 & $1.58167 \times 10^{9}$ & $1.52484 \times 10^{9}$ & $1.49088 \times 10^{9}$ \\
50 & $1.63121 \times 10^{15}$ & $1.59625 \times 10^{15}$ & $1.57467 \times 10^{15}$ \\
\hline
\end{tabular}

Tabela 3.2: Valores da constante $C_{r}$ para A (3.32).

\begin{tabular}{|c|ccc|}
\hline \multirow{2}{*}{$n_{1}=n_{2}$} & \multicolumn{3}{|c|}{$\mathbf{r}$} \\
\cline { 2 - 4 } & $1 / 2$ & 1 & $3 / 2$ \\
\hline \hline 10 & 0.03128 & 0.02781 & 0.02606 \\
30 & $2.79006 \times 10^{-9}$ & $2.68979 \times 10^{-9}$ & $2.62995 \times 10^{-9}$ \\
50 & $4.01676 \times 10^{-15}$ & $3.93053 \times 10^{-15}$ & $3.87783 \times 10^{-15}$ \\
\hline
\end{tabular}

Tabela 3.3: Valor médio de A para dados gerados segundo $M_{2}$. 
Através dos resultados obtidos na simulação, verificamos que o $C_{\mathrm{r}}$ é totalmente absorvido por $\frac{(R S S)^{-\frac{n}{2}}}{\prod_{j=1}^{2}\left(R S S_{j}\right)^{-\frac{n_{1}}{2}}}$, que é a contribuição da variância no fator de Bayes a posteriori (3.32).

Podemos então concluir que apesar de $C_{\tau}$ depender fortemente de n (ver Tabela 3.2), o fator de Bayes a posteriori (3.32) não foi afetado pelo paradoxo de Lindley; pelo contrário, quanto maior o tamanho da amostra, maior foi a sua conviç̧ão em aceitar o modelo $M_{2}$. 


\section{Capítulo 4}

\section{Comparação de dois Modelos com Erros nas Variáveis}

\subsection{Introdução}

A teoria de estimação com erros nas variáveis vem sendo discutida com grande intensidade nestes últimos anos. Como referência básica para estas discussões, citamos o artigo recente de Sprent (1990). Entretanto, existe na literatura um número pequeno de trabalhos referentes a testes de hipótese. Creasy (1956), Bansal (1987) e Fuller (1987) discutem o teste de hipótese a respeito da inclinação do modelo. Bansal (1990), Wong (1991), Bolfarine, Nascimento e Rodrigues (1991) discutem o problema de comparar várias inclinações em modelos com erros nas variáveis. Neste capítulo iremos comparar os coeficientes de dois modelos de regressão com erros nas variáveis (2.2) através do fator de Bayes a posteriori.

Utilizamos o modelo condicional (2.3) onde consideramos o caso em que o fator de atenuação $K_{j}$ é conhecido, sendo que ele pode ser igual 
$\left(K_{1}=K_{2}\right)$ e diferente $\left(K_{1} \neq K_{2}\right)$, e também o caso em que o fator de atenuação $K_{j}$ é desconhecido. Finalizaremos com uma ilustração numérica.

\subsection{Fator de Atenuação Conhecido}

Nesta seção, nós assumimos que $o$ fator de atenuação $K_{j}=\frac{\sigma_{s}^{2}}{\sigma_{s}^{2}+\sigma_{u j}^{2}}$, para $j=1,2$ é conhecido e consideramos o modelo condicional definido em (2.3), ou seja,

$$
Y_{i j} / X_{i j} \sim N \quad\left[\begin{array}{llll}
\left.K_{j} X_{i j} \beta_{j}, \lambda_{j}\right] .
\end{array}\right.
$$

Consideremos as seguintes notações:

$$
\begin{aligned}
& Y_{j}^{\prime}=\left(Y_{1 j}, \ldots, Y_{n, j}\right) \\
& Z_{j}^{\prime}=K_{j} X_{j}^{\prime}=\left(K_{j} X_{1 j}, \ldots, K_{j} X_{n j}\right)=\left(Z_{1 j}, \ldots, Z_{n, j}\right) \\
& \underset{\sim}{Y}=\left[\begin{array}{l}
Y_{1} \\
Y_{2}
\end{array}\right] \\
& Z=\left[\begin{array}{ll}
Z_{1} & 0_{1} \\
0_{2} & Z_{2}
\end{array}\right] \\
& 0_{j}^{\prime}=(\underbrace{0, \cdots, 0}_{n_{j}}) \\
& V=\left[\begin{array}{cc}
V_{1} & 0 \\
0 & V_{2}
\end{array}\right] \\
& \underline{\alpha}=\left(\begin{array}{l}
\beta_{1} \\
\beta_{2}
\end{array}\right) \\
& 1^{\prime}=(1,1)
\end{aligned}
$$

onde $\mathrm{V}$ é uma matriz de bloco diagonal com $V_{j}=\lambda_{j} I_{j}$ e $I_{j}$ é a matriz 
identidade de dimensão $n_{j}$.

Para o modelo $M_{1}$, onde a hipótese é $H_{0}: \beta_{1}=\beta_{2}=\beta$, temos que a distribuição condicional, utilizando a notação acima, é $\underset{\sim}{Y} / Z \sim N[Z 1 \beta, V]$. Para o parâmetro $\Theta_{1}=\left(\beta, \lambda_{1}, \lambda_{2}\right)$ assumimos a priori não informativa da forma:

$$
\Pi\left(\Theta_{1}\right) \propto \frac{1}{\lambda_{1}^{r} \lambda_{2}^{r}} .
$$

Para $r=\frac{1}{2}, 1 e \frac{3}{2},(4.1)$ é uma priori difusa .

A função de verossimilhança é dada por:

$$
L_{1}\left(\Theta_{1}\right) \propto \frac{1}{\lambda_{1}^{\frac{n_{1}}{2}} \lambda_{2}^{\frac{n_{2}}{2}}} \exp \left\{-\frac{1}{2} \sum_{j=1}^{2} \frac{1}{\lambda_{j}} \sum_{i=1}^{n_{j}}\left(Y_{i j}-\beta Z_{i j}\right)^{2}\right\} .
$$

Sob o modelo $M_{2}$ que corresponde $H_{1}: \beta_{1} \neq \beta_{2}$, com distribuição condicional $\underset{\sim}{Y} / Z \sim N[Z \underline{\beta}, V]$, temos o parâmetro $\Theta_{2}=\left(\begin{array}{llll}\beta_{1}, & \beta_{2}, \lambda_{1}, \lambda_{2}\end{array}\right)$, para o qual consideramos a priori (4.1) e a função de verossimilhança sendo,

$$
L_{2}\left(\Theta_{2}\right) \propto \frac{1}{\lambda_{1}^{\frac{n_{1}}{2}} \lambda_{2}^{\frac{n_{2}}{2}}} \exp \left\{-\frac{1}{2} \sum_{j=1}^{2} \frac{1}{\lambda_{j}} \sum_{i=1}^{n_{j}}\left(Y_{i j}-\beta_{j} Z_{i j}\right)^{2}\right\} .
$$

\section{Lema 5}

Sob o modelo $M_{1}$ com o fator de atenuação $K_{j}$ conhecido, temos que 


$$
\bar{L}_{1}^{A}=\left[\prod_{j=1}^{2} \frac{\Gamma\left(n_{j}+r-1\right)}{\Gamma\left(\frac{n_{1}}{2}+r-1\right)}\left(R S S_{j}\right)^{-\frac{n_{1}}{2}} 2^{-\left(\frac{n_{1}}{2}+r-1\right)}\right] 2^{-\frac{1}{2}} e^{-S / 2}
$$

onde $R S S_{j}$ é dado em (4.8) e $S$ em (4.10).

Prova:

Combinando (4.1) e (4.2), obtemos:

$$
\begin{aligned}
l_{k}^{(1)} & \propto \int \frac{1}{\lambda_{1}^{\frac{k n_{1}}{2}+r} \lambda_{2}^{\frac{k n_{2}}{2}+r}} \exp \left\{-\frac{k}{2} \sum_{j=1}^{2} \frac{1}{\lambda_{j}} \sum_{i=1}^{n_{j}}\left(Y_{i j}-\beta Z_{i j}\right)^{2}\right\} d \Theta_{1} \\
& =\int\left[\prod_{j=1}^{2} \int \frac{1}{\lambda_{j}^{\frac{k n_{j}}{2}+r}} \exp \left\{-\frac{k}{2 \lambda_{j}} \sum_{i=1}^{n_{j}}\left(Y_{i j}-\beta Z_{i j}\right)^{2}\right\} d \lambda_{j}\right] d \beta
\end{aligned}
$$

para $k=1,2$.

Integrando (4.5) em relação a $\lambda_{j}$, usando a fórmula da integral dada no Apêndice 1.a, obtemos:

$l_{k}^{(1)} \propto \int \prod_{j=1}^{2}\left[\frac{k}{2} \sum_{i=1}^{n_{j}}\left(Y_{i j}-\beta Z_{i j}\right)^{2}\right]^{-\left(\frac{k n_{j}}{2}+r-1\right)} \Gamma\left(\frac{k n_{j}}{2}+r-1\right) d \beta$.

Se fizermos as mesmas consideraçōes de (3.21), obtemos: 
$l_{k}^{(1)} \propto\left[\prod_{j=1}^{2} \Gamma\left(\frac{k n_{j}}{2}+r-1\right)\left(\frac{k R S S_{j}}{2}\right)^{-\left(\frac{k n_{j}}{2}+r-1\right)}\right] \int e^{-\sum_{j=1}^{2}\left(\frac{k n_{j}}{2}+r-1\right) \frac{k_{j}^{2} \sum_{i=1}^{n_{j}} x_{i j}^{2}\left(\theta-\hat{\hat{\beta}}_{j}\right)^{2}}{k S S_{j}}} d \beta$

onde

$$
\begin{gathered}
\hat{\hat{\beta}}_{j}=K_{j}^{-1} \frac{\sum_{i=1}^{n_{j}} Y_{i j} X_{i j}}{\sum_{i=1}^{n_{j}} X_{i j}^{2}} e \\
R S S_{j}=\sum_{i=1}^{n_{j}}\left(Y_{i j}-\hat{\hat{\beta}}_{j} Z_{i j}\right)^{2} .
\end{gathered}
$$

Utilizando o método de Laplace (ver A pêndice 2) para o cálculo de integrais e integrando (4.6) em relação a $\beta$, obtemos:

$$
\begin{aligned}
l_{k}^{(1)} \propto & {\left[\prod_{j=1}^{2} \Gamma\left(\frac{k n_{j}}{2}+r-1\right)\left(\frac{k R S S_{j}}{2}\right)^{-\left(\frac{k n_{j}}{2}+r-1\right)}\right] \frac{\sqrt{2 \pi}}{\sqrt{k \sum_{j=1}^{2} a_{j}}} . } \\
& . \exp \left\{-\frac{k S}{2}\right\} \exp \left\{-\sum_{j=1}^{2}(r-1) \frac{K_{j}^{2} \sum_{i=1}^{n_{j}} X_{i j}^{2}\left(\widehat{\beta}-\hat{\widehat{\beta}}_{j}\right)^{2}}{R S S_{j}}\right\}
\end{aligned}
$$

onde

$$
\begin{aligned}
& S=\sum_{j=1}^{2} a_{j}\left(\hat{\beta}-\hat{\hat{\beta}}_{j}\right)^{2}, \\
& \hat{\beta}=\frac{\sum_{j=1}^{2} a_{j} \hat{\hat{\beta}}_{j}}{\sum_{j=1}^{2} a_{j}} e
\end{aligned}
$$




$$
a_{j}=\frac{n_{j} K_{j}^{2} \sum_{i=1}^{n_{j}} X_{i j}^{2}}{R S S_{j}} .
$$

Fazendo $k=1,2 \mathrm{em}(4.9)$, temos respectivamente,

$$
\begin{aligned}
& l_{1}^{(1)} \propto\left[\prod_{j=1}^{2} \Gamma\left(\frac{n_{j}}{2}+r-1\right)\left(\frac{R S S_{j}}{2}\right)^{-\left(\frac{n_{2}}{2}+r-1\right)}\right] \frac{\sqrt{2 \pi}}{\sqrt{\sum_{j=1}^{2} a_{j}}} . \\
& . \exp \left\{-\frac{S}{2}\right\} \exp \left\{-\sum_{j=1}^{2}(r-1) \frac{K_{j}^{2} \sum_{i=1}^{n,} X_{i j}^{2}\left(\hat{\beta}-\hat{\bar{\beta}}_{j}\right)^{2}}{R S S_{j}}\right\} e \\
& l_{2}^{(1)} \propto\left[\prod_{j=1}^{2} \Gamma\left(n_{j}+r-1\right)\left(R S S_{j}\right)^{-(n j+r-1)}\right] \frac{\sqrt{2 \pi}}{\sqrt{2 \sum_{j=1}^{2} a_{j}}} . \\
& . \exp \{-S\} \quad \exp \left\{-\sum_{j=1}^{2}(r-1) \frac{K_{j}^{2} \sum_{i=1}^{n_{j}} X_{i j}^{2}\left(\hat{\beta}-\hat{\widehat{\beta}}_{j}\right)^{2}}{R S S_{j}}\right\} \text {. }
\end{aligned}
$$

Portanto, a média a posteriori é dada por:

$$
\bar{L}_{1}^{A}=\frac{l_{2}^{(1)}}{l_{1}^{(1)}}=\left[\prod_{j=1}^{2} \frac{\Gamma\left(n_{j}+r-1\right)}{\Gamma\left(\frac{n_{j}}{2}+r-1\right)}\left(R S S_{j}\right)^{-\frac{n_{j}}{2}} 2^{-\left(\frac{n_{j}}{2}+r-1\right)}\right] 2^{-\frac{1}{2}} e^{-S / 2} .
$$

\section{Lema 6}

Sob o modelo $M_{2}$ com fator de atenuação $K_{j}$ conhecido temos que 
$\bar{L}_{2}^{A}=\prod_{j=1}^{2} \frac{\Gamma\left(n_{j}+r-1\right)}{\Gamma\left(\frac{n_{2}}{2}+r-1\right)}\left(R S S_{j}\right)^{-\frac{n_{3}}{2}} 2^{-\left(\frac{n_{1}}{2}+r-1\right)} 2^{-\frac{2}{2}}\left(\frac{n_{j}+r-1}{n_{j}+2 r-2}\right)^{-\frac{1}{2}}$,

onde $R S S_{j}$ é dado em (4.8).

Prova:

Combinando (4.1) e (4.3), obtemos:

$$
\begin{aligned}
l_{k}^{(2)} & \propto \int \frac{1}{\lambda_{1}^{\frac{k n_{1}}{2}+r} \lambda_{2}^{\frac{k n_{2}}{2}+r}} \exp \left\{-\frac{k}{2} \sum_{j=1}^{2} \frac{1}{\lambda_{j}} \sum_{i=1}^{n_{j}}\left(Y_{i j}-\beta_{j} Z_{i j}\right)^{2}\right\} d \Theta_{2} \\
& =\int\left[\prod_{j=1}^{2} \int \frac{1}{\lambda_{j}^{\frac{k n_{j}}{2}+r}} \exp \left\{-\frac{k}{2 \lambda_{j}} \sum_{i=1}^{n_{1}}\left(Y_{i j}-\beta_{j} Z_{i j}\right)^{2}\right\} d \lambda_{j}\right] d \beta_{j}
\end{aligned}
$$

para $\mathbf{k}=1,2$.

Integrando (4.14) com respeito a $\lambda_{j}$, utilizando o mesmo procedimento de (4.5), obtemos:

$$
l_{k}^{(2)} \propto \prod_{j=1}^{2} \Gamma\left(\frac{k n_{j}}{2}+r-1\right)\left(\frac{k R S S_{j}}{2}\right)^{-\left(\frac{k n_{j}}{2}+r-1\right)} \int e^{-\left(\frac{k n_{j}}{2}+r-1\right) \frac{\sum_{i=1}^{n_{j}} z_{i j}^{2}\left(\beta_{j}-\hat{\widehat{\beta}}_{j}\right)^{2}}{R S S_{j}}} d \beta_{j} .
$$

Integrando a expressão acima em relação a $\beta_{j}$ e aproximando a normal, obtemos: 
$l_{k}^{(2)} \propto \prod_{j=1}^{2} \Gamma\left(\frac{k n_{j}}{2}+r-1\right)\left(\frac{k R S S_{j}}{2}\right)^{-\left(\frac{k n_{2}}{2}+r-1\right)} \sqrt{2 \pi}\left(\frac{R S S_{j}}{\left(k n_{j}+2 r-2\right) \sum_{i=1}^{n_{j}} Z_{i j}^{2}}\right)^{\frac{1}{2}}$.

Fazendo $k=1,2$, em (4.15), temos respectivamente,

$l_{1}^{(2)} \propto \prod_{j=1}^{2} \Gamma\left(\frac{n_{j}}{2}+r-1\right)\left(\frac{R S S_{j}}{2}\right)^{-\left(\frac{n_{j}}{2}+r-1\right)} \sqrt{2 \pi}\left(\frac{R S S_{j}}{\left(n_{j}+2 r-2\right) \sum_{i=1}^{n_{j}} Z_{i j}^{2}}\right)^{\frac{1}{2}} e$

$l_{2}^{(2)} \propto \prod_{j=1}^{2} \Gamma\left(n_{j}+r-1\right)\left(R S S_{j}\right)^{-\left(n_{j}+r-1\right)} \sqrt{2 \pi}\left(\frac{R S S_{j}}{\left(2 n_{j}+2 r-2\right) \sum_{i=1}^{n_{j}} Z_{i j}^{2}}\right)^{\frac{1}{2}}$.

Portanto, a média a posteriori é dada por:

$\bar{L}_{2}^{A}=\frac{l_{2}^{(2)}}{l_{1}^{(2)}}=\prod_{j=1}^{2} \frac{\Gamma\left(n_{j}+r-1\right)}{\Gamma\left(\frac{n_{j}}{2}+r-1\right)}\left(R S S_{j}\right)^{-\frac{n_{j}}{2}} 2^{-\left(\frac{n_{j}}{2}+r-1\right)} 2^{-\frac{1}{2}}\left(\frac{n_{j}+r-1}{n_{j}+2 r-2}\right)^{-\frac{1}{2}}$

\section{Teorema 4}

O fator de Bayes a posteriori para o modelo (2.2) com o fator de atenuaçâo $K_{j}$ conhecido é dado por:

$$
A=\left[\prod_{j=1}^{2} \frac{n_{j}+r-1}{n_{j}+2 r-2}\right]^{\frac{1}{2}} 2^{1 / 2} e^{-s / 2}
$$

onde $S$ é dado por (4.10). 
Prova:

Segue basicamente do Lema 5 e Lema 6 e da definição de fator de Bayes a posteriori.

\subsubsection{Comentários Importantes:}

1. Sendo $-2 \ln A=S-\ln 2 C_{r}$ onde $C_{r}=\left[\prod_{j=1}^{2} \frac{n_{j}+r-1}{n_{j}+2 r-2}\right]$, novamente, $-2 \ln A$ pertence a família da verossimilhança penalizada proposta por Smith e Spiegelhalter (1980) para $\nu=1$ e $m=\ln 2 C_{r}$ (ver Apêndice 3). Quando $r=1$, temos que $C_{r}=1$ e $m=\ln 2$, o qual coincide com o resultado da Seção 3 de Aitkin (1991).

2. Considerando o coeficiente $a_{j}$ dado por (4.12), isto é $a_{j}=\frac{K_{i}^{2} \sum_{i=1}^{n_{j}} X_{i j}^{2}}{R S S_{j} / n_{j}}$, onde $\frac{R S S_{j}}{n_{j}}$ é a estimativa da variância de cada população, ou seja $\hat{\lambda}_{j}=\frac{R S S_{j}}{n_{j}}$, então $a_{j}$ pode ser representado por $a_{j}=\frac{1}{\hat{\lambda}_{j} / K_{j}^{2} \sum_{i=1}^{n_{j}} X_{i j}^{2}}=$ $=\frac{1}{\widehat{\operatorname{Var}}\left(\hat{\hat{\beta}}_{j}\right)}$, que é o inverso da variância estimada de $\hat{\widehat{\hat{\beta}}}_{j}$ ou a medida de informação de Fisher estimada.

3. $\operatorname{Em~(4.7)~} \widehat{\hat{\beta}}_{j}$ é o estimador corrigido de $\beta_{j}$ e em (4.11) $\hat{\beta}$ é a média dos estimadores corrigidos ponderado pelo $a_{j}$ citado no item anterior. Assim a população com mais informação terá maior peso em $\hat{\beta}$.

4. Em (4.10) $S$ é a estatística que compara o estimador corrigido de cada população $\left(\hat{\hat{\beta}}_{j}\right)$ com o estimador comum de $\beta(\widehat{\beta})$, ponderado pelo $a_{j} \cdot$ 
5. Nota-se que o fator de Bayes a posteriori obtido através do modelo condicionado coincide com o fator de Bayes a posteriori usando o modelo não condicionado, apresentado no minicurso - O Modelo de Regressão com Erros nas Variáveis - pag.82.

\subsection{Fator de Atenuação Desconhecido e Iguais}

Assumindo que o fator de atenuação $K_{j}=\frac{\sigma_{s, j}^{2}}{\sigma_{s_{j}}^{2}+\sigma_{u j}^{2}}$, para $j=1,2$ é desconhecido e igual, podemos representar $K_{j}$ e $\beta_{j}$, ambos desconhecidos, por $\phi_{j}=K_{j} \beta_{j}$ no modelo condicional definido em (2.3). Assim sendo, obtemos:

$$
Y_{i j} / X_{i j} \sim N\left[\phi_{j} X_{i j} \quad, \quad \lambda_{j}\right]
$$

Para $K_{j}$ desconhecido e igual testar a hipótese $H_{0}: \beta_{1}=\beta_{2}=\beta$ é o mesmo que testar $H_{0}: \phi_{1}=\phi_{2}=\phi$, onde a distribuição condicional para o modelo $M_{1}$ passa a ser $\underset{\sim}{Y} / X \sim N[X 1 \phi, V]$. Para o parâmetro $\Theta_{1}=\left(\phi, \lambda_{1}, \lambda_{2}\right)$, adotamos a priori não informativa (4.1) e a função de verossimilhança como sendo,

$$
L_{1}\left(\Theta_{1}\right) \propto \frac{1}{\lambda_{1}^{\frac{n_{1}}{2}} \lambda_{2}^{\frac{n_{2}}{2}}} \exp \left\{-\frac{1}{2} \sum_{j=1}^{2} \frac{1}{\lambda_{j}} \sum_{i=1}^{n_{j}}\left(Y_{i j}-\phi X_{i j}\right)^{2}\right\}
$$


Sob o modelo $M_{2}$ que corresponde $H_{1}: \phi_{1} \neq \phi_{2}$, com distribuição condicional $\underset{\sim}{Y} / X \sim N[X \underset{\sim}{\phi}, V]$, temos o parãmetro $\Theta_{2}=\left(\phi_{1}, \phi_{2}, \lambda_{1}, \lambda_{2}\right)$ para o qual consideramos a priori (4.1) e a função de verossimilhança sendo,

$$
L_{2}\left(\Theta_{2}\right) \propto \frac{1}{\lambda_{1}^{\frac{n_{1}}{2}} \lambda_{2}^{\frac{n_{2}}{2}}} \exp \left\{-\frac{1}{2} \sum_{j=1}^{2} \frac{1}{\lambda_{j}} \sum_{i=1}^{n_{j}}\left(Y_{i j}-\phi_{j} X_{i j}\right)^{2}\right\} .
$$

\section{Lema 7}

Sob o modelo $M_{1}$ com o fator de atenuação $K_{j}$ desconhecido, temos que

$$
\bar{L}_{1}^{A}=\left[\prod_{j=1}^{2} \frac{\Gamma\left(n_{j}+r-1\right)}{\Gamma\left(\frac{n_{j}}{2}+r-1\right)}\left(R S S_{j}\right)^{-\frac{n_{i}}{2}} 2^{-\left(\frac{n_{j}}{2}+r-1\right)}\right] 2^{-\frac{1}{2}} e^{-S / 2}
$$

onde $R S S_{j}$ é dado em (4.27).

Prova:

Combinando (4.1) e (4.18), obtemos:

$$
\begin{aligned}
l_{k}^{(1)} & \propto \int \frac{1}{\lambda_{1}^{\frac{k n_{1}}{2}+r} \lambda_{2}^{\frac{k n_{2}}{2}+\tau}} \exp \left\{-\frac{k}{2} \sum_{j=1}^{2} \frac{1}{\lambda_{j}} \sum_{i=1}^{n_{j}}\left(Y_{i j}-\phi X_{i j}\right)^{2}\right\} d \Theta_{1} \\
& =\int\left[\prod_{j=1}^{2} \int \frac{1}{\lambda_{j}^{\frac{k n_{j}}{2}+r}} \exp \left\{-\frac{k}{2 \lambda_{j}} \sum_{i=1}^{n_{j}}\left(Y_{i j}-\phi X_{i j}\right)^{2}\right\} d \lambda_{j}\right] d \phi
\end{aligned}
$$


$\operatorname{para} k=1,2$.

Se em (3.20) substituirmos $\beta$ por $\phi e \sigma_{j}^{2}$ por $\lambda_{j}$, teremos a mesma expressão. Então, utilizando os mesmos procedimentos de (3.20) para calcular as integrais, obtemos:

$$
\begin{aligned}
l_{k}^{(1)} \propto & {\left[\prod_{j=1}^{2} \Gamma\left(\frac{k n_{j}}{2}+r-1\right)\left(\frac{k R S S_{j}}{2}\right)^{-\left(\frac{k n_{j}}{2}+r-1\right)}\right] \frac{\sqrt{2 \pi}}{\sqrt{k \sum_{j=1}^{2} a_{j}}} } \\
& \cdot \exp \left\{-\frac{k S}{2}\right\} \exp \left\{-\sum_{j=1}^{2}(r-1) \frac{\sum_{i=1}^{n} X_{i j}^{2}\left(\hat{\phi}-\hat{\phi}_{j}\right)^{2}}{R S S_{j}}\right\},
\end{aligned}
$$

onde

$$
\begin{aligned}
S & =\sum_{j=1}^{2} a_{j}\left(\hat{\phi}-\hat{\phi}_{j}\right)^{2}, \\
\hat{\phi} & =\frac{\sum_{j=1}^{2} a_{j} \hat{\phi}_{j}}{\sum_{j=1}^{2} a_{j}}, \\
\hat{\phi}_{j} & =\frac{\sum_{i=1}^{n_{j}} Y_{i j} X_{i j}}{\sum_{i=1}^{n_{j}} X_{i j}^{2}}, \\
a_{j} & =\frac{n_{j} \sum_{i=1}^{n_{j}} X_{i j}^{2}}{R S S_{j}} e \\
R S S_{j} & =\sum_{i=1}^{n_{j}}\left(Y_{i j}-\hat{\phi}_{j} X_{i j}\right)^{2} .
\end{aligned}
$$

Fazendo $\mathrm{k}=1,2 \mathrm{em}(4.22)$, e calculando a média a posteriori, obtemos: 


$$
\bar{L}_{1}^{A}=\frac{l_{2}^{(1)}}{l_{1}^{(1)}}=\left[\prod_{j=1}^{2} \frac{\Gamma\left(n_{j}+r-1\right)}{\Gamma\left(\frac{n_{1}}{2}+r-1\right)}\left(R S S_{j}\right)^{-\frac{n_{1}}{2}} 2^{-\left(\frac{n_{1}}{2}+r-1\right)}\right] 2^{-\frac{1}{2}} e^{-S / 2}
$$

\section{Lema 8}

Sob o modelo $M_{2}$ com fator de atenuação $K_{j}$ desconhecido, temos que

$$
\bar{L}_{2}^{A}=\prod_{j=1}^{2} \frac{\Gamma\left(n_{j}+r-1\right)}{\Gamma\left(\frac{n_{j}}{2}+r-1\right)}\left(R S S_{j}\right)^{-\frac{n_{j}}{2}} 2^{-\left(\frac{n_{j}}{2}+r-1\right)} 2^{-\frac{1}{2}}\left(\frac{n_{j}+r-1}{n_{j}+2 r-2}\right)^{-\frac{1}{2}} .
$$

onde $R S S_{j}$ é dado em (4.27).

\section{Prova:}

Combinando (4.1) e (4.19), obtemos:

$$
\begin{aligned}
l_{k}^{(2)} & \propto \int \frac{1}{\lambda_{1}^{\frac{k n_{1}}{2}+r} \lambda_{2}^{\frac{k n_{2}}{2}+r}} \exp \left\{-\frac{k}{2} \sum_{j=1}^{2} \frac{1}{\lambda_{j}} \sum_{i=1}^{n_{j}}\left(Y_{i j}-\phi_{j} X_{i j}\right)^{2}\right\} d \Theta_{2} \\
& =\int\left[\prod_{j=1}^{2} \int \frac{1}{\lambda_{j}^{\frac{k n_{j}}{2}+r}} \exp \left\{-\frac{k}{2 \lambda_{j}} \sum_{i=1}^{n_{j}}\left(Y_{i j}-\phi_{j} X_{i j}\right)^{2}\right\} d \lambda_{j}\right] d \phi_{j}
\end{aligned}
$$

$$
\text { para } k=1,2
$$


Se em (3.29) substituirmos $\beta_{j}$ por $\phi_{j}$ e $\sigma_{j}^{2}$ por $\lambda_{j}$, teremos a mesma expressão. Utilizando os mesmos procedimentos usado em (3.29) para calcular as integrais, obtemos:

$l_{k}^{(2)} \propto \prod_{j=1}^{2} \Gamma\left(\frac{k n_{j}}{2}+r-1\right)\left(\frac{k R S S_{j}}{2}\right)^{-\left(\frac{k n_{2}}{2}+r-1\right)} \sqrt{2 \pi}\left(\frac{R S S_{j}}{\left(k n_{j}+2 r-2\right) \sum_{i=1}^{n_{j}} X_{i j}^{2}}\right)^{\frac{1}{2}}$.

Fazendo $k=1,2$, em (4.30), calculando a média a posteriori, obtemos:

$\bar{L}_{2}^{A}=\frac{l_{2}^{(2)}}{l_{1}^{(2)}}=\prod_{j=1}^{2} \frac{\Gamma\left(n_{j}+r-1\right)}{\Gamma\left(\frac{n_{j}}{2}+r-1\right)}\left(R S S_{j}\right)^{-\frac{n_{2}}{2}} 2^{-\left(\frac{n_{2}}{2}+r-1\right)} 2^{-\frac{1}{2}}\left(\frac{n_{j}+r-1}{n_{j}+2 r-2}\right)^{-\frac{1}{2}}$

\section{Teorema 5}

O fator de Bayes a posteriori para o modelo (2.2) com o fator de atenuação $K_{j}$ desconhecido é dado por:

$$
A=\left[\prod_{j=1}^{2} \frac{n_{j}+r-1}{n_{j}+2 r-2}\right]^{\frac{1}{2}} 2^{1 / 2} e^{-s / 2}
$$

onde $S$ é dado por (4.23).

Prova:

Segue basicamente dos Lemas 7 e 8 e da definição de fator de Bayes a posteriori. 
Nota-se que o fator de Bayes a posteriori (4.31) coincide com o fator de Bayes a posteriori (3.31) encontrado na seção 3.3. Assim sendo, as interpretações são as mesmas da referida seção.

\subsection{Ilustração Numérica}

Nesta seção apresentamos uma ilustração numérica, via método de Monte Carlo, com a finalidade de verificar o comportamento do fator de Bayes a posteriori dado em (4.16).

Como para A (4.16) o fator de atenuação é conhecido, podemos considerar dois casos: o primeiro em que o fator de atenuação $K_{j}$ é conhecido e igual para $\mathrm{j}=1,2$ e o segundo, em que o fator de atenuação $K_{j}$ é conhecido e diferente para $\mathrm{j}=1,2$.

$\underline{\text { Para } K_{j} \text { conhecido e iguais : }}$

O procedimento da simulação é o mesmo do Capitulo 3, ou seja foram geradas 50 amostras baseado no modelo (2.2) com $n_{1}=n_{2}=10,30$ e 50 ; $\beta_{1}=\beta_{2}=1 ; K_{1}=K_{2}=0.5,0.8$ e 1 com respectivos valores $\sigma_{e_{j}}^{2}=\sigma_{u_{j}}^{2}=1 ; \sigma_{e_{j}}^{2}=1$ e $\sigma_{u_{j}}^{2}=0.25 ; \quad \sigma_{e_{j}}^{2}=1$ e $\sigma_{u_{j}}^{2}=0$ e $x_{i j} \sim N(0,1)$ para os três valores de $K_{j}$. Para cada amostra gerada foi calculado o fator de Bayes a posteriori (4.16). Este procedimento foi repetido 50 vezes, calculando-se a média de $\mathbf{A}$. Os valores encontrados são apresentados na Tabela 4.1 


\begin{tabular}{|c|c|c|c|c|}
\hline \multirow[b]{2}{*}{$n_{j}$} & \multirow[b]{2}{*}{$K_{1}=K_{2}$} & \multicolumn{3}{|c|}{$\mathbf{r}$} \\
\hline & & $1 / 2$ & 1 & $3 / 2$ \\
\hline 10 & $\begin{array}{c}0.5 \\
0.8 \\
1\end{array}$ & $\begin{array}{l}0.9528 \\
0.9388 \\
1.0321\end{array}$ & $\begin{array}{l}0.9026 \\
0.8894 \\
0.9777\end{array}$ & $\begin{array}{l}0.8616 \\
0.8489 \\
0.9333\end{array}$ \\
\hline 30 & $\begin{array}{c}0.5 \\
0.8 \\
1\end{array}$ & $\begin{array}{l}1.0518 \\
1.0671 \\
0.9837\end{array}$ & $\begin{array}{l}1.0339 \\
1.0489 \\
0.9670\end{array}$ & $\begin{array}{l}1.0173 \\
1.0320 \\
0.9514\end{array}$ \\
\hline 50 & $\begin{array}{c}0.5 \\
0.8 \\
1\end{array}$ & $\begin{array}{l}1.0990 \\
0.9976 \\
1.0707\end{array}$ & $\begin{array}{l}1.0879 \\
0.9876 \\
1.0599\end{array}$ & $\begin{array}{l}1.0773 \\
0.9779 \\
1.0496\end{array}$ \\
\hline
\end{tabular}

Tabela 4.1: Valor médio de A para valores de $K_{j}$ iguais

\section{Para $K_{j}$ conhecido e diferentes :}

Aqui repetimos o procedimento anterior mudando apenas o valor do fator de atenuação $K_{j}$, que é diferente para cada j. Os valores considerados foram os seguintes:

$$
\begin{array}{llllll}
K_{1}=0.5 & \Longrightarrow & \sigma_{e_{1}}^{2}=1, & \sigma_{u_{1}}^{2}=1 & \text { e } & \sigma_{x_{1}}^{2}=1 ; \\
K_{2}=0.8 & \Longrightarrow & \sigma_{e_{2}}^{2}=1, & \sigma_{u_{2}}^{2}=0.25 & \text { e } & \sigma_{x_{2}}^{2}=1 e \\
K_{1}=0.6 & \Longrightarrow & \sigma_{e_{1}}^{2}=1, & \sigma_{u_{1}}^{2}=0.67 & \text { e } & \sigma_{x_{1}}^{2}=1 ; \\
K_{2}=0.75 & \Longrightarrow & \sigma_{e_{2}}^{2}=1, & \sigma_{u_{2}}^{2}=0.33 & \text { e } & \sigma_{x_{2}}^{2}=1 .
\end{array}
$$

$O$ resultado desta simulação é apresentado na Tabela 4.2. 


\begin{tabular}{|c|c|c|c|c|}
\hline \multirow[b]{2}{*}{$n_{j}$} & \multirow[b]{2}{*}{$K_{1} \neq K_{2}$} & \multicolumn{3}{|c|}{$\mathbf{r}$} \\
\hline & & $1 / 2$ & 1 & $3 / 2$ \\
\hline \multirow[t]{2}{*}{10} & $\begin{array}{l}0.5 \\
0.8\end{array}$ & 1.0888 & 1.0315 & 0.9846 \\
\hline & $\begin{array}{c}0.6 \\
0.75\end{array}$ & 1.0747 & 1.0181 & 0.9718 \\
\hline \multirow[t]{2}{*}{30} & $\begin{array}{l}0.5 \\
0.8\end{array}$ & 1.0866 & 1.0682 & 1.0509 \\
\hline & $\begin{array}{c}0.6 \\
0.75\end{array}$ & 1.0069 & 0.9543 & 0.9113 \\
\hline \multirow[t]{2}{*}{50} & $\begin{array}{l}0.5 \\
0.8 \\
\end{array}$ & 0.9876 & 0.9777 & 0.9681 \\
\hline & $\begin{array}{c}0.6 \\
0.75\end{array}$ & 1.0193 & 1.0090 & 0.9991 \\
\hline
\end{tabular}

Tabela 4.2: Para valores de $K_{j}$ diferentes

Com base nos dados das Tabelas 4.1 e 4.2 , verificamos que tanto para erros iguais como para erros diferentes, detecta-se com bastante convicção o modelo verdadeiro $\left(M_{1}\right)$. Também verificamos que em relação a priori é estável. Assim sendo, podemos concluir que o fator de Bayes a posteriori dado em (4.16) é insensível em relação a priori e ao erro.

Quanto ao tamanho da amostra verificamos que não existe alteração, ou seja, o fator de Bayes a posteriori não aumentou quando aumentamos o tamanho da amostra. Não sendo afetado pelo tamanho da amostra, evita o paradoxo de Lindley. 


\section{Capítulo 5}

\section{Teste da Razão de Verossimilhança}

\subsection{Introdução}

Até o momento, comparamos os coeficientes de dois modelos de regressão linear assumindo algumas situações. No capítulo 3 o modelo considerado foi o de regressão sem erro nas variáveis (2.1), levando em conta uma primeira etapa em que as variâncias são iguais e desconhecidas, uma segunda etapa em que são desiguais e desconhecidas e por fim comparamos simultaneamente os coeficientes e as variâncias de dois modelos. No capítulo 4 comparamos os coeficientes de dois modelos de regressão com erros nas variáveis (2.2), considerando os casos em que o fator de atenuação $\left(K_{j}\right)$ é conhecido e desconhecido. Tudo isso usando uma estatística desenvolvida em cima da metodologia Bayesiana.

Neste capítulo, formulamos uma análise clássica para comparar coeficientes em modelos de regressão, supondo para o modelo (2.1), que as 
variâncias são iguais e desconhecidas. Esta análise clássica baseia-se no conhecido teste da razão de verossimilhança $(\lambda)$.

Finalizaremos este capítulo com um estudo comparativo entre o teste da razão de verossimilhança ( $\lambda$ ) e o fator de Bayes a posteriori ( $A$ ), usando para isso, a escala de Aitkin, o teste qui-quadrado e o teste $F$.

\subsection{Teste da Razão de Verossimilança Generalizado}

Nesta seção definimos o teste da razão de verossimilhança de uma maneira geral. Considerando uma família geral de distribuição $f\left(x ; \theta_{1}, \theta_{2}, \ldots, \theta_{k}\right)$, a hipótese para ser testada será chamada de hipótese nula, denotada por $H_{0}$, que consiste nos parâmetros que pertencem a algum subspaço $\omega$ do espaço paramétrico $\Omega$. A hipótese alternativa será denotada por $H_{1}$.

Seja $x_{1}, x_{2}, \ldots, x_{n}$ uma amostra de tamanho $n$ para uma população com densidade $f\left(x ; \theta_{1}, \theta_{2}, \ldots, \theta_{k}\right)$, onde $\Omega$, o espaço paramétrico, é o conjunto de todos os valores de $\left(\theta_{1}, \theta_{2}, \ldots, \theta_{k}\right)$.

Sobre esta amostra, desejamos testar a hipótese : $H_{0}:\left(\theta_{1}, \theta_{2}, \ldots, \theta_{k}\right)$ é um ponto em $\omega$ contra a hipótese alternativa : $H_{1}:\left(\theta_{1}, \theta_{2}, \ldots, \theta_{k}\right)$ é um ponto em $\bar{\omega}$, onde $\bar{\omega}$ é $\Omega-\omega$. 
A verossimilhança da amostra é:

$$
L=\prod_{i=1}^{n} f\left(x_{i} ; \theta_{1}, \theta_{2}, \ldots, \theta_{k}\right)
$$

A verossimilhança como uma função dos parâmetros, usualmente tem um máximo quando os parâmetros variam sobre todo o espaço paramétrico $\Omega$; representaremos este máximo valor por $L\left(\hat{\theta}_{1}, \hat{\theta}_{2}, \ldots, \hat{\theta}_{k}\right)$, ou simplesmente $L(\hat{\Omega})$. No subspaço $\omega, \mathrm{L}$ usualmente também tem um valor máximo, que representaremos por $L(\hat{\omega})$.

Desta forma, a definição do teste da razão de verossimilhança generalizada é o quociente:

$$
\lambda=\frac{L(\hat{\omega})}{L(\hat{\Omega})}
$$

\subsection{Teste da Razão de Verossimilhança para Mode- los Lineares}

O interesse desta seção, é formular um teste usando a metodologia clássica nas mesmas situações em que foi criado o fator de Bayes a posteriori da seção 3.2. Assim sendo, assumiremos o modelo definido na seção 3.2, ou seja: 


$$
\begin{aligned}
Y_{i j}=\beta_{j} X_{i j}+e_{i j} \quad, i & =1, \ldots, n_{j} \\
j & =1,2
\end{aligned}
$$$$
e_{i j} \sim N\left(0, \sigma^{2}\right) \text {. }
$$

A função de verossimilhança é dada por:

$L\left(\beta_{1}, \beta_{2}, \sigma^{2}\right)=\frac{1}{\left(2 \pi \sigma^{2}\right)^{\frac{n}{2}}} \exp \left\{-\frac{1}{2 \sigma^{2}} \sum_{j=1}^{2} \sum_{i=1}^{n_{j}}\left(Y_{i j}-\beta_{j} X_{i j}\right)^{2}\right\}$,

onde $n=n_{1}+n_{2}$.

\section{Teorema 6}

$O$ teste da razão de verossimilhança para testar a hipótese $H_{0}: \beta_{1}=\beta_{2}=\beta$ contra a hipótese alternativa $H_{1}: \beta_{1} \neq \beta_{2}$ é dado por:

$$
\lambda=\left[\frac{\sum_{j=1}^{2} \sum_{i=1}^{n_{j}}\left(Y_{i j}-\hat{\beta}_{j} X_{i j}\right)^{2}}{\sum_{j=1}^{2} \sum_{i=1}^{n_{j}}\left(Y_{i j}-\hat{\beta} X_{i j}\right)^{2}}\right]^{n / 2},
$$

onde

$$
\begin{aligned}
\hat{\beta}_{j} & =\frac{\sum_{i=1}^{n_{j}} Y_{i j} X_{i j}}{\sum_{i=1}^{n_{j}} X_{i j}^{2}} e \\
\hat{\beta} & =\frac{\sum_{j=1}^{2} \sum_{i=1}^{n_{j}} Y_{i j} X_{i j}}{\sum_{j=1}^{2} \sum_{i=1}^{n_{j}} X_{i j}^{2}} .
\end{aligned}
$$


Prova:

Sob a hipótese $H_{0}$ temos que a função de verossimilhança é dada por:

$$
L(\omega)=L\left(\beta, \sigma^{2}\right)=\frac{1}{\left(2 \pi \sigma^{2}\right)^{\frac{n}{2}}} \exp \left\{-\frac{1}{2 \sigma^{2}} \sum_{j=1}^{2} \sum_{i=1}^{n_{j}}\left(Y_{i j}-\beta X_{i j}\right)^{2}\right\}
$$

Geralmente é mais fácil trabalhar com o logaritmo da função de verossimilhança para se obter o seu máximo, pois os valores de $\beta$ e $\sigma^{2}$, que maximizam - logaritmo da função de verossimilhança, também maximizam a função de verossimilhança. O logaritmo da função de verossimilhança (5.2) para $\beta$ e $\sigma^{2}$ (da parte informativa) é dada por:

$$
l\left(\beta, \sigma^{2}\right)=-\frac{n}{2} \ln \sigma^{2}-\frac{1}{2 \sigma^{2}} \sum_{j=1}^{2} \sum_{i=1}^{n_{j}}\left(Y_{i j}-\beta X_{i j}\right)^{2} .
$$

As primeiras derivadas de (5.3) em relação a $\beta$ e $\sigma^{2}$ são dadas por,

$$
\begin{aligned}
& \frac{\partial l\left(\beta, \sigma^{2}\right)}{\partial \beta}=\frac{1}{\sigma^{2}} \sum_{j=1}^{2} \sum_{i=1}^{n_{j}}\left(Y_{i j}-\beta X_{i j}\right)\left(X_{i j}\right) e \\
& \frac{\partial l\left(\beta, \sigma^{2}\right)}{\partial \sigma^{2}}=-\frac{n}{2 \sigma^{2}}+\frac{1}{2\left(\sigma^{2}\right)^{2}} \sum_{j=1}^{2} \sum_{i=1}^{n_{j}}\left(Y_{i j}-\beta X_{i j}\right)^{2},
\end{aligned}
$$

respectivamente. 
Portanto, igualando a zero as expressões (5.4) e (5.5) e resolvendo as equações, encontramos os estimadores de máxima verossimilhança de $\beta$ e $\sigma^{2}$ dados por:

$$
\begin{aligned}
\hat{\beta} & =\frac{\sum_{j=1}^{2} \sum_{i=1}^{n} Y_{i j} X_{i j}}{\sum_{j=1}^{2} \sum_{i=1}^{n,} X_{i j}^{2}} e \\
\widehat{\sigma^{2}} & =\frac{\sum_{j=1}^{2} \sum_{i=1}^{n,}\left(Y_{i j}-\hat{\beta} X_{i j}\right)^{2}}{n} .
\end{aligned}
$$

Substituindo $\hat{\beta}$ e $\widehat{\sigma^{2}}$ em (5.2) e cancelando termos semelhantes, encontramos a função de máxima verossimilhança dada por:

$$
L(\widehat{\omega})=L\left(\widehat{\beta}, \widehat{\sigma^{2}}\right)=(2 \pi)^{-n / 2}\left[\frac{n}{\sum_{j=1}^{2} \sum_{i=1}^{n_{j}}\left(Y_{i j}-\widehat{\beta} X_{i j}\right)^{2}}\right]^{\frac{n}{2}} \exp \left\{-\frac{n}{2}\right\} \cdot(5
$$

Para a hipótese alternativa $\left(H_{1}: \beta_{1} \neq \beta_{2}\right)$, temos que a função de verossimilhança é dada por:

$$
L(\Omega)=L\left(\beta_{1}, \beta_{2}, \sigma^{2}\right)=\frac{1}{\left(2 \pi \sigma^{2}\right)^{\frac{n}{2}}} \exp \left\{-\frac{1}{2 \sigma^{2}} \sum_{j=1}^{2} \sum_{i=1}^{n_{j}}\left(Y_{i j}-\beta_{j} X_{i j}\right)^{2}\right\}
$$

Por razões explicadas anteriormente, é recomendável trabalhar com o logaritmo da função de verossimilhança. Assim o logaritmo da função de verossimilhança (da parte informativa) para $\beta_{1}, \beta_{2}$ e $\sigma^{2}$ é:

$$
l\left(\beta_{1}, \beta_{2}, \sigma^{2}\right)=-\frac{n}{2} \ln \sigma^{2}-\frac{1}{2 \sigma^{2}} \sum_{j=1}^{2} \sum_{i=1}^{n_{j}}\left(Y_{i j}-\beta_{j} X_{i j}\right)^{2} .
$$


Derivando (5.10) com respeito $\beta_{j}$ e $\sigma^{2}$ e igualando zero, isto é, $\partial l / \partial \beta_{j}=0$ e $\partial l / \partial \sigma^{2}=0$, obtemos os estimadores de máxima verossimilhança de $\beta_{j}$ e $\sigma^{2}$ dados por:

$$
\begin{aligned}
& \hat{\beta}_{j}=\frac{\sum_{i=1}^{n,} Y_{i j} X_{i j}}{\sum_{i=1}^{n_{j}} X_{i j}^{2}} e \\
& \widehat{\sigma^{2}}=\frac{\sum_{j=1}^{2} \sum_{i=1}^{n,}\left(Y_{i j}-\hat{\beta}_{j} X_{i j}\right)^{2}}{n} .
\end{aligned}
$$

Substituindo $\hat{\beta}_{j} \quad e \quad \widehat{\sigma^{2}}$ em (5.9) e arrumando a expressão, encontramos a função de máxima verossimilhança dada por:

$$
L(\widehat{\Omega})=L\left(\hat{\beta}_{j}, \widehat{\sigma^{2}}\right)=(2 \pi)^{-n / 2}\left[\frac{n}{\sum_{j=1}^{2} \sum_{i=1}^{n_{j}}\left(Y_{i j}-\hat{\beta}_{j} X_{i j}\right)^{2}}\right]^{\frac{n}{2}} \exp \left\{-\frac{n}{2}\right\}
$$

Achando o quociente entre (5.8) e (5.13), eliminando os termos semelhantes e arrumando a expressão, encontramos o teste da razão de verossimilhança que é dado por:

$$
\lambda=\left[\frac{\sum_{j=1}^{2} \sum_{i=1}^{n_{j}}\left(Y_{i j}-\hat{\beta}_{j} X_{i j}\right)^{2}}{\sum_{j=1}^{2} \sum_{i=1}^{n_{j}}\left(Y_{i j}-\hat{\beta} X_{i j}\right)^{2}}\right]^{n / 2} .
$$




\subsection{Comparação do Teste da Razão de Verossimi- lhança com o Fator de Bayes a Posteriori}

O objetivo desta seção é comparar via simulação o teste da razão de verossimilhança com o fator de Bayes a posteriori.

Nos capítulos anteriores não estavamos comparando o fator de Bayes a posteriori com nenhum teste, isto é, apenas nos interessava verificar se, em média, ele detectava a hipótese, usando para isso a escala proposta por Aitkin (1991). Entretanto nesta seção, com a finalidade de compará-lo com outro teste, vamos proceder de maneira diferente.

Primeiramente vamos considerar a escala de evidência, sugerida por Aitkin (1991), que rejeita $H_{0}$ para valores menores que $1 / 20$. Depois usaremos convenientemente uma transformação para $\lambda e A$ definida por $\Lambda=-2 \ln \lambda$ e $\Lambda_{A}=-2 \ln A$ que tem, assintoticamente, distribuição $\chi_{1}^{2}(0.05)$ com grau de liberdade um e nível de significância 0.05. Finalizaremos com o teste $\mathbf{F}$ (Seber, 1977 ) onde $F_{\lambda}=\frac{n-p}{q}\left[\lambda^{-2 / n}-1\right] e$ $F_{A}=\frac{n-p}{q}\left[A^{-2 / n}-1\right]$ têm distribuição $F_{q, n-p}$, com $q=1$ e $n-p=n-2$ graus de liberdade.

Foram geradas 500 amostras de acordo com o modelo, para $n_{1}=n_{2}=10$ à $100 ; \beta_{1}=\beta_{2}=1 ; \sigma^{2}=1 ; X_{i 1} \sim N(30,2) e$ $X_{i 2} \sim N(40,2)$. Para cada amostra gerada, calculamos o $A$ (3.15) e o $\lambda(5.1)$ e comparamos com a escala de Aitkin. Depois calculamos o $\Lambda_{A}$ e $\Lambda$ e comparamos com $\chi_{1}^{2}=3.841$ e por último, calculamos 0 $F_{A} e F_{\lambda}$ e comparamos com $F_{1, n-2}$. A Tabela 5.1 apresenta em porcen- 
tagem o número de vezes (entre as 500) que $H_{0}: \beta_{1}=\beta_{2}$ (verdadeira) foi rejeitada, usando cada uma das situações acima, que são: ( A e $\lambda$ ) $<0.05$; $\left(\Lambda_{A}\right.$ e $\left.\Lambda\right)>3.841$ e $\left(F_{A}\right.$ e $\left.F_{\lambda}\right)>F_{1, n-2}$.

\begin{tabular}{|c|ccc|ccc|}
\hline & & FBP & & & TRV & \\
$n_{1}=n_{2}$ & A & $\Lambda_{A}$ & $F_{A}$ & $\lambda$ & $\Lambda$ & $F_{\lambda}$ \\
\hline \hline & & & & & & \\
\hline 10 & $3.6 \%$ & $6.6 \%$ & $5.0 \%$ & $3.2 \%$ & $\mathbf{8 . 0} \%$ & $\mathbf{5 . 6 \%}$ \\
20 & $1.8 \%$ & $4.4 \%$ & $4.4 \%$ & $2.0 \%$ & $5.8 \%$ & $\mathbf{5 . 4} \%$ \\
30 & $1.2 \%$ & $4.0 \%$ & $3.8 \%$ & $1.2 \%$ & $5.6 \%$ & $4.6 \%$ \\
40 & $1.0 \%$ & $3.0 \%$ & $3.0 \%$ & $1.0 \%$ & $4.2 \%$ & $3.8 \%$ \\
50 & $0.8 \%$ & $3.6 \%$ & $3.0 \%$ & $1.0 \%$ & $5.4 \%$ & $5.0 \%$ \\
60 & $2.0 \%$ & $4.6 \%$ & $4.4 \%$ & $2.4 \%$ & $6.0 \%$ & $5.8 \%$ \\
70 & $1.2 \%$ & $3.6 \%$ & $3.4 \%$ & $1.4 \%$ & $5.2 \%$ & $5.2 \%$ \\
80 & $0.6 \%$ & $3.4 \%$ & $3.4 \%$ & $1.0 \%$ & $4.4 \%$ & $4.2 \%$ \\
90 & $1.0 \%$ & $4.8 \%$ & $4.6 \%$ & $1.4 \%$ & $5.8 \%$ & $5.4 \%$ \\
100 & $1.2 \%$ & $3.8 \%$ & $3.6 \%$ & $1.6 \%$ & $6.0 \%$ & $5.8 \%$ \\
& & & & & & \\
\hline
\end{tabular}

Tabela 5.1: Número de rejeição de $H_{0}$ em porcentagem. 
Utilizando os valores da Tabela 5.1 construímos os gráficos. Na Figura 5.1 temos o gráfico do tamanho da amostra versus $A$ e $\lambda$; na Figura 5.2 temos o gráfico do tamanho da amostra versus $\Lambda_{A}$ e $\Lambda$ e na Figura 5.3, o gráfico do tamanho da amostra versus $F_{A} e F_{\lambda}$.

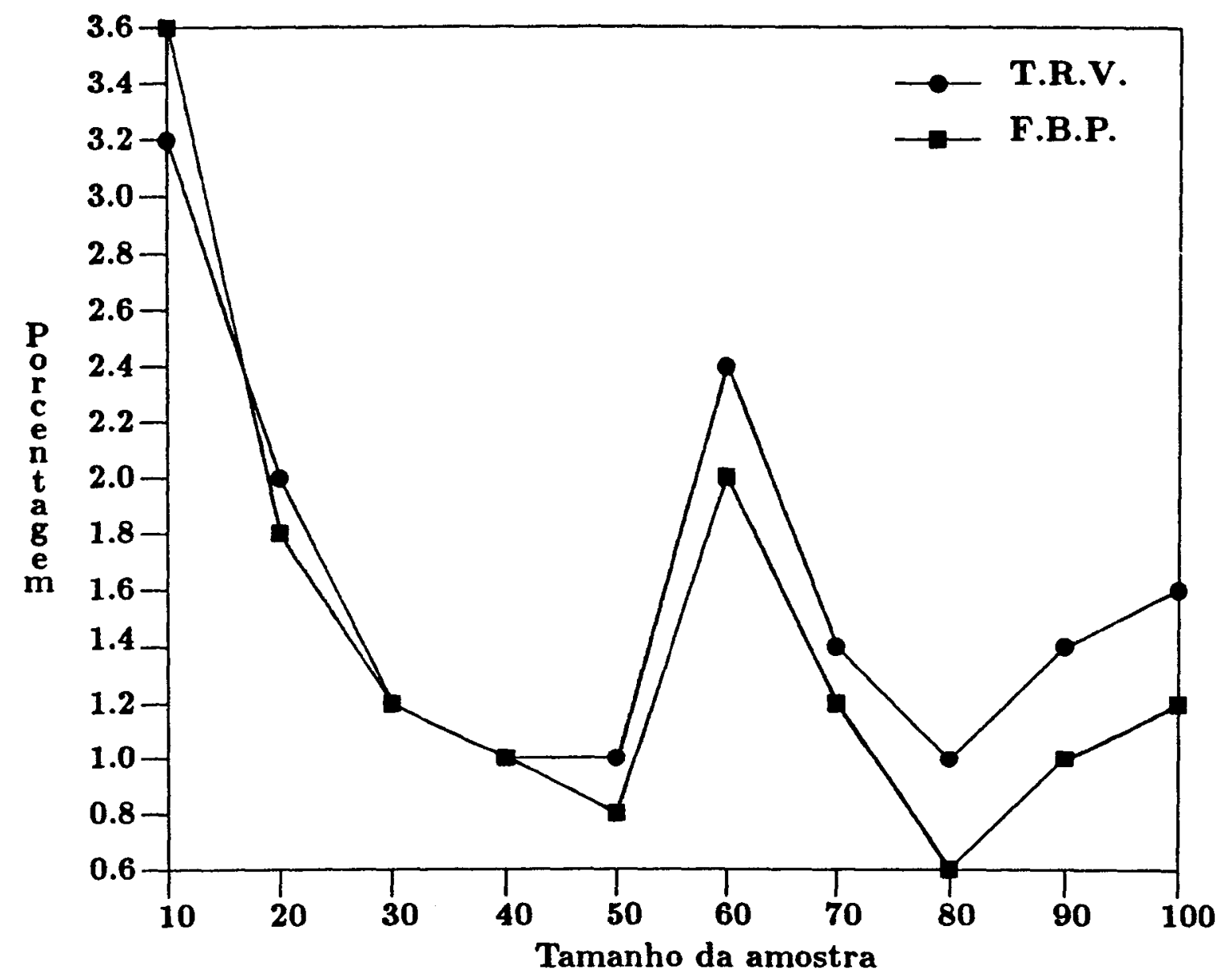

Figura 5.1: Gráfico do Tamanho da Amostra versus a porcentagem do teste A e $\lambda$. 


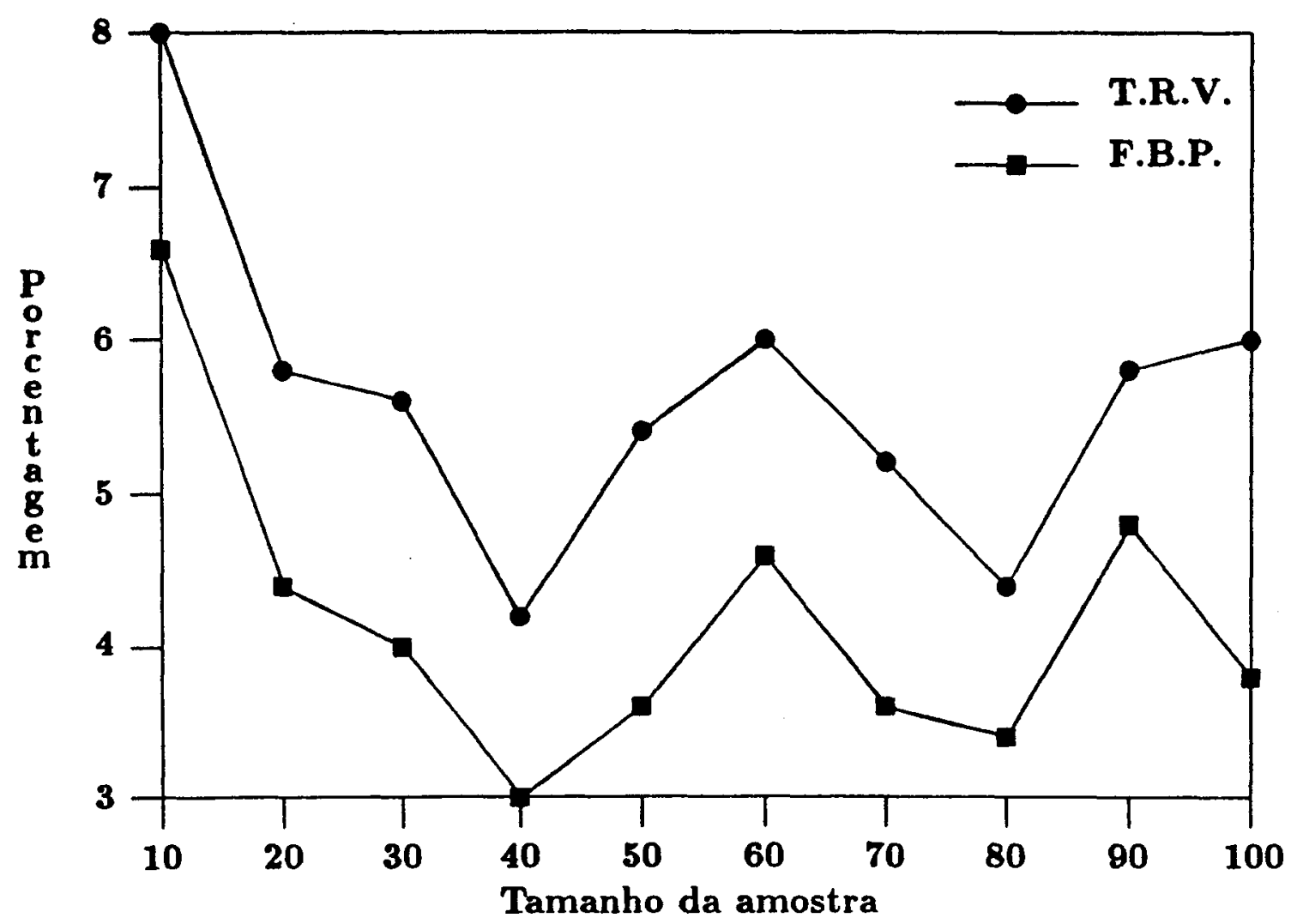

Figura 5.2: Gráfico do Tamanho da amostra versus a porcentagem dos testes $\Lambda_{A} e \Lambda$.

Nota-se da Figura 5.1, que o fator de Bayes a posteriori só perde para o teste da razão de verossimilhança, quando o tamanho da amostra é 10 . Nas demais ele detecta mais o modelo verdadeiro do que o teste da razão de verossimilhança. Verificamos também que tanto na Figura 5.2 como na Figura 5.3, o fator de Bayes a posteriori da mais suporte à hipótese $H_{0}$ do que o teste da razão de verossimilhança, mantendo-se na taxa de rejeição abaixo do nivel do teste (percentil de $95 \%$ da distribuição). 


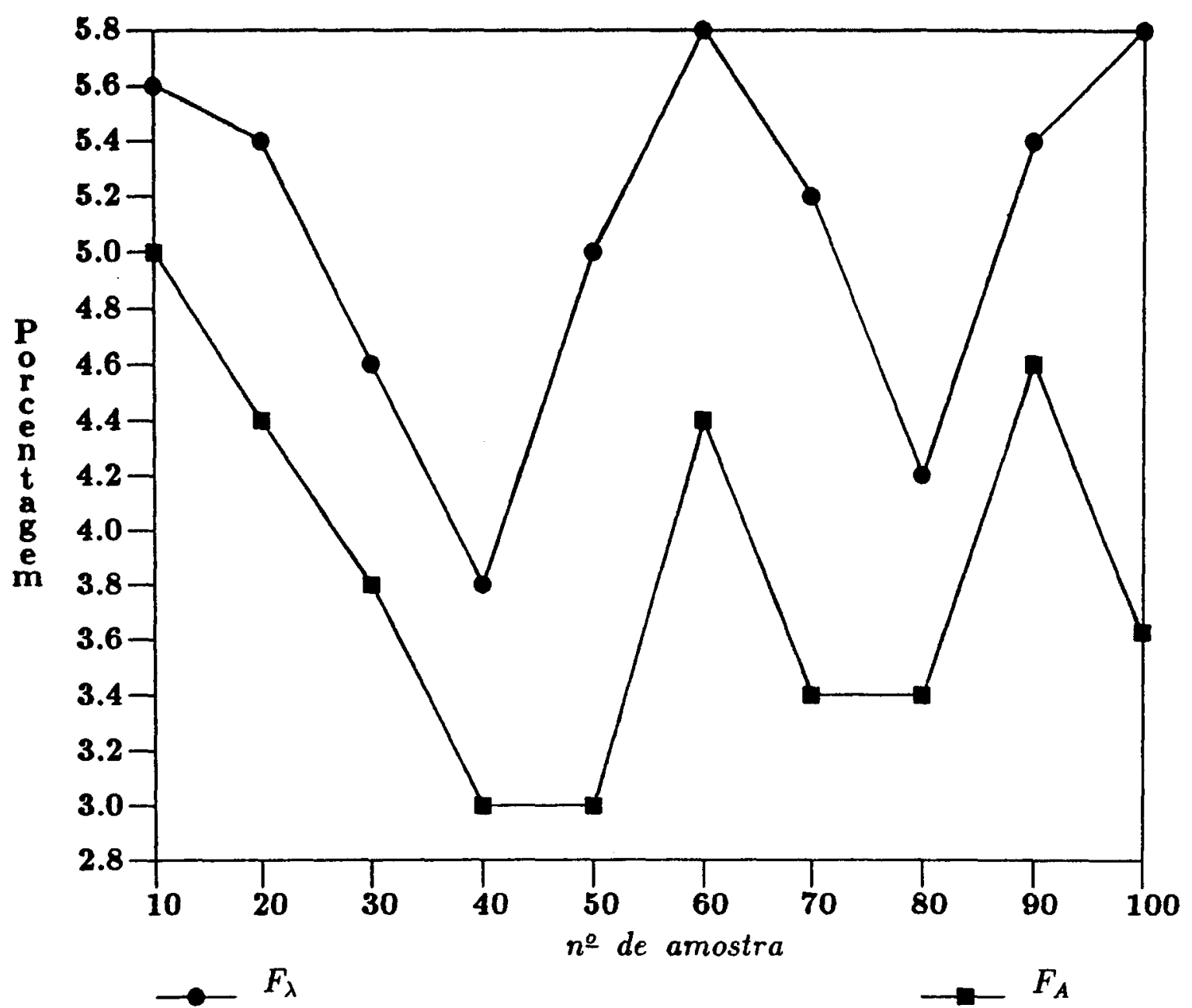

Figura 5.3: Gráfico do Tamanho da Amostra versus a porcentagem dos testes $F_{\lambda}$ e $F_{A}$.

Assim sendo, concluímos que a escala de Aitkin só deve ser usada com o teste da razão de verossimilhança, quando a amostra é pequena ( $n \leq 10$ ); caso contrário, usar o fator de Bayes a posteriori. Entretanto, quando consideramos os testes $\chi^{2}$ e F, o emprego do fator de Bayes a posteriori é mais recomendado, uma vez que supera o teste da razão de verossimilhança em qualquer tamanho de amostra. 


\section{Capítulo 6}

\section{Teste da Razão de Entropia a Posteriori}

\subsection{Introdução}

Utilizando a metodologia Bayesiana em capitulos anteriores, comparamos modelos através do fator de Bayes a posteriori, considerando priori não informativa e explorando o uso do método de Laplace para aproximar integrais.

Neste capítulo, propomos um outro método exato para comparar modelos, também com enfoque Bayesiano, e baseado na entropia proposta por Shannon (1948), que chamamos de teste da razão de entropia a posteriori, cuja notação será $A_{v}$. Iremos supor o caso em que as variâncias são iguais e desconhecidas.

Apresentamos também um estudo comparativo entre o fator de Bayes a posteriori e o teste da razão de entropia a posteriori, usando ilustrações 
numéricas via método de Monte Carlo, com a utilização da escala de evidência proposta por Jeffrey (1961) para avaliar o fator de Bayes (B).

\subsection{Formulação do Teste da Razão de Entropia a Posteriori}

Baseado no princípio da verossimilhança vamos definir uma medida de informação dos dados a respeito de $\Theta$, como sendo $I_{v}=\int L(Y / \Theta) \ln L(Y / \Theta) d Y$, ou seja, a média do logaritmo da função de verossimilhança.

Percebemos que $I_{v}$ é a medida de entropia negativa, que vem sendo usada por Zellner (1984), e esta medida coincide com a de Shannon (1948) com sinal trocado.

Como $I_{v}$ vai depender dos parâmetros que são desconhecidos, vamos eliminá-los tomando a esperança a posteriori da medida de informação $I_{v}$. Assim sendo, vamos definir o teste da razão de entropia a posteriori, como o quociente de médias de entropia negativa em relação a posteriori, ou seja,

$$
A_{v}=\frac{E_{\Theta_{2}}\left[I_{v_{2}}\right]}{E_{\Theta_{1}}\left[I_{v_{1}}\right]}=\frac{I_{2}^{A_{v}}}{I_{1}^{A_{v}}}
$$

onde

$$
I_{j}^{A_{v}}=E_{\Theta_{j}}\left[\int L\left(Y / \Theta_{j}\right) \ln L\left(Y / \Theta_{j}\right) d Y\right], \text { para } j=1,2
$$




\subsection{Comparação de Modelos via Teste da Razão de Entropia a Posteriori}

Como o nosso objetivo é o mesmo da seção 3.2, ou seja comparar dois modelos de regressão com variâncias iguais e desconhecidas apenas mudando a estatística do teste, vamos considerar as mesmas notações e suposições anteriores. Os lemas que demonstraremos a seguir, serão importantes para obter o teste da razão de entropia a posteriori.

Lema 9

Sob o modelo $M_{2}$ com $\sigma_{1}^{2}=\sigma_{2}^{2}=\sigma^{2}$, temos que

$$
I_{2}^{A_{v}}=C-n\left[\ln S_{2}+\frac{1}{2 \nu_{2}}\right],
$$

onde

$$
\begin{aligned}
& C=-\frac{1}{2}[n \ln (2 \pi)+1], \\
& S_{2}^{2}=\frac{\sum_{j=1}^{2} \sum_{i=1}^{n_{j}}\left(Y_{i j}-\hat{\beta}_{j} X_{i j}\right)^{2}}{\nu_{2}}, \\
& \hat{\beta}_{j}=\frac{\sum_{i=1}^{n_{j}} Y_{i j} X_{i j}}{\sum_{i=1}^{n_{j}} X_{i j}^{2}} e \\
& \nu_{2}=n-2 .
\end{aligned}
$$


Prova:

A função de verossimilhança sob o modelo $M_{2}$ é dada por:

$$
L_{2}\left(\Theta_{2}\right)=\frac{1}{\left(2 \pi \sigma^{2}\right)^{\frac{n}{2}}} \exp \left\{-\frac{1}{2 \sigma^{2}} \sum_{j=1}^{2} \sum_{i=1}^{n_{j}}\left(Y_{i j}-\beta_{j} X_{i j}\right)^{2}\right\}
$$

Logaritmando a expressão acima, obtemos:

$$
\ln L_{2}\left(\Theta_{2}\right)=-\frac{n}{2} \ln (2 \pi)-n \ln \sigma-\frac{1}{2 \sigma^{2}} \sum_{j=1}^{2} \sum_{i=1}^{n_{j}}\left(Y_{i j}-\beta_{j} X_{i j}\right)^{2} .
$$

Multiplicando a expressão (6.7) com a expressão (6.8) e integrando, obtemos:

$$
\begin{aligned}
I_{v_{2}} & =\int L_{2}\left(\Theta_{2}\right) \ln L_{2}\left(\Theta_{2}\right) d Y \\
& =-\frac{n}{2} \ln (2 \pi)-n \ln \sigma-\frac{1}{2} \\
& =C-n \ln \sigma,
\end{aligned}
$$

onde C é dado em (6.3).

Tomando a esperança a posteriori da expressão acima, obtemos:

$$
I_{2}^{A_{v}}=E_{\theta_{2}}\left[I_{v_{2}}\right]=C-n E[\ln \sigma],
$$


onde,

$$
E[\ln \sigma]=\int_{0}^{\infty} \ln \sigma \Pi(\sigma / \text { dados }) d \sigma
$$

Substituindo $\Pi$ ( $\sigma /$ dados ) (posteriori marginal de $\sigma$ ) em (6.10), obtemos:

$$
E[\ln \sigma]=\int_{0}^{\infty} \ln \sigma \frac{2}{\Gamma\left(\frac{\nu_{2}}{2}\right)}\left(\frac{\nu_{2} S_{2}^{2}}{2}\right)^{\frac{\nu_{2}}{2}} \frac{1}{\sigma^{\nu_{2}+1}} \exp \left\{-\frac{\nu_{2} S_{2}^{2}}{2 \sigma^{2}}\right\} d \sigma,
$$

onde $S_{2}^{2}$ e $\nu_{2}$ é dado em (6.4) e (6.6), respectivamente.

Para facilitar a resolução de (6.11), vamos fazer a seguinte mudança de variável:

$$
\begin{array}{rlrl}
x & =\frac{\nu_{2} S_{2}^{2}}{2 \sigma^{2}} & \rightarrow & \sigma=\sqrt{\frac{\nu_{2} S_{2}^{2}}{2}} \frac{1}{x^{1 / 2}} e \\
\frac{d \sigma}{d x}=-\frac{x^{-1 / 2}}{2 x} \sqrt{\frac{\nu_{2} S_{2}^{2}}{2}} & \rightarrow & \left|\frac{d \sigma}{d x}\right|=\sqrt{\frac{\nu_{2} S_{2}^{2}}{2}} \frac{x^{-3 / 2}}{2} .
\end{array}
$$

Fazendo esta mudança de variável em (6.11) e após algumas simplificações algébricas, obtemos:

$$
\begin{aligned}
E[\ln \sigma] & =\int_{0}^{\infty} \ln \left(\sqrt{\frac{\nu_{2} S_{2}^{2}}{2}} x^{-1 / 2}\right) \frac{x^{\frac{\nu_{2}}{2}-1}}{\Gamma\left(\frac{\nu_{2}}{2}\right)} \exp \{-x\} d x \\
& =\ln \sqrt{\frac{\nu_{2} S_{2}^{2}}{2}}-\frac{1}{2} \int_{0}^{\infty} \ln x \frac{x^{\frac{\nu_{2}}{2}-1}}{\Gamma\left(\frac{\nu_{2}}{2}\right)} \exp \{-x\} d x .
\end{aligned}
$$


Para resolver a integral em (6.12), partimos da definição de uma função Digama que é $\psi(\alpha)=\frac{\partial \ln \Gamma(\alpha)}{\partial \alpha}$, onde $\Gamma(\alpha)=\int x^{\alpha-1} e^{-x} d x$ (função gama). É possível derivar sobre o sinal de integração, sendo assim $\psi(\alpha)=\frac{1}{\Gamma(\alpha)} \int x^{\alpha-1} \ln x e^{-x} d x$, coincidindo com a integral de (6.12) para $\alpha=\frac{n_{2}}{2}$. Deste modo,

$$
E[\ln \sigma]=\ln \sqrt{\frac{\nu_{2} S_{2}^{2}}{2}}-\frac{1}{2} \psi\left(\frac{\nu_{2}}{2}\right)
$$

onde $\psi\left(\frac{\aleph^{2}}{2}\right)$ é uma função Digama.

Considerando $\psi\left(\frac{\nu_{2}}{2}\right) \approx \ln \left(\frac{\nu_{2}}{2}\right)-\frac{1}{\nu_{2}}($ Abramowitz e Stegun ) e substituindo na expressão acima, obtemos:

$$
\begin{aligned}
E[\ln \sigma] & =\ln \sqrt{\frac{\nu_{2} S_{2}^{2}}{2}}-\frac{1}{2}\left[\ln \frac{\nu_{2}}{2}-\frac{1}{\nu_{2}}\right] \\
& =\ln S_{2}+\frac{1}{2 \nu_{2}}
\end{aligned}
$$

Substituindo o resultado de (6.13) em (6.9), obtemos:

$$
I_{2}^{A_{v}}=C-n\left[\ln S_{2}+\frac{1}{2 \nu_{2}}\right] \text {. }
$$

\section{Lema 10}

Sob o modelo $M_{1}$ com $\sigma_{1}^{2}=\sigma_{2}^{2}=\sigma^{2}$, temos que 


$$
I_{1}^{A_{v}}=C-n\left[\ln S_{1}+\frac{1}{2 \nu_{1}}\right]
$$

onde Cé dado em (6.3) e

$$
\begin{aligned}
S_{1}^{2} & =\frac{\sum_{j=1}^{2} \sum_{i=1}^{n j}\left(Y_{i j}-\hat{\beta} X_{i j}\right)^{2}}{\nu_{1}}, \\
\hat{\beta} & =\frac{\sum_{j=1}^{2} \sum_{i=1}^{n_{j}} Y_{i j} X_{i j}}{\sum_{j=1}^{2} \sum_{i=1}^{n_{j}} X_{i j}^{2}} e \\
\nu_{1} & =n-1 .
\end{aligned}
$$

Prova:

A função de verossimilhança sob o modelo $M_{1}$ é dada por:

$$
L_{1}\left(\Theta_{1}\right)=\frac{1}{\left(2 \pi \sigma^{2}\right)^{\frac{n}{2}}} \exp \left\{-\frac{1}{2 \sigma^{2}} \sum_{j=1}^{2} \sum_{i=1}^{n j}\left(Y_{i j}-\beta X_{i j}\right)^{2}\right\}
$$

Logaritmando a expressão acima, obtemos:

$$
\ln L_{1}\left(\Theta_{1}\right)=-\frac{n}{2} \ln (2 \pi)-n \ln \sigma-\frac{1}{2 \sigma^{2}} \sum_{j=1}^{2} \sum_{i=1}^{n_{j}}\left(Y_{i j}-\beta X_{i j}\right)^{2} .
$$

Portanto, procedendo de forma análoga a demonstração do Lema 9, o resultado é facilmente obtido. 


\section{Teorema 7}

$O$ teste da razão de entropia a posteriori para o modelo (2.1) com $\sigma_{1}^{2}=\sigma_{2}^{2}=\sigma^{2} \dot{e}$ dado por:

$$
A_{v}=\frac{C-n\left[\ln S_{2}+\frac{1}{2 \nu_{2}}\right]}{C-n\left[\ln S_{1}+\frac{1}{2 \nu_{1}}\right]},
$$

onde $S_{1}, \nu_{1}$ é dado por (6.15) (6.17) , e $S_{2}, \nu_{2}$ é dado por (6.4) (6.6).

Prova:

Dos Lemas 9 e 10 e da definição do teste da razão de entropia a posteriori (6.1), o resultado é diretamente obtido.

\subsection{Comparação do Fator de Bayes a Posteriori com o Teste da Razão de Entropia a Posteriori}

Nesta seção pretendemos comparar o fator de Bayes a posteriori, considerando $(r=1)$ já que o fator de Bayes a posteriori é insensível em relação a priori, com o teste da razão de entropia a posteriori utilizando para 
isso a simulação e uma escala de evidência usada por Jeffrey (1961) para avaliar o fator de Bayes (B), a qual mostraremos a seguir:

Escala de Evidència para Avaliar o Fator de Bayes

(Jeffrey, 1961)

$\begin{array}{rlll} & \mathrm{B}>1 & \Longrightarrow & \text { Apoia } M_{1} \\ 10^{-1 / 2}<\mathrm{B}<1 & \Longrightarrow & \text { Fraca evidência contra } M_{1} \\ 10^{-1}<\mathrm{B}<10^{-1 / 2} & \Longrightarrow & \text { Moderada evidência contra } M_{1} \\ 10^{-2}<\mathrm{B}<10^{-1} & \Longrightarrow & \text { Forte evidência contra } M_{1} \\ \mathrm{~B}<10^{-2} & \Longrightarrow & \text { Decisiva evidência contra } M_{1}\end{array}$

Para simular, vamos proceder da mesma maneira que vinhamos procedendo em capítulos anteriores, ou seja, vamos gerar dados primeiramente para $n_{1}=n_{2}=10,30$ e $50 ; \beta_{1}=\beta_{2}=1 ; \sigma^{2}=1 e$ $X_{i j} \sim N(30,2)$. Após gerarmos as amostras, calculamos o fator de Bayes a posteriori e o teste da razão de entropia a posteriori, e verificamos em que intervalo da escala de evidência acima, ele se encontra. Repetimos este procedimento 500 vezes e achamos a porcentagem de cada intervalo. Os resultados obtidos são apresentados na Tabela 6.1. 


\begin{tabular}{|c|c|c|c|}
\hline$n_{1}=n_{2}$ & escala & $\mathbf{A}$ & $A_{v}$ \\
\hline 10 & $\begin{array}{c}B>1 \\
10^{-1 / 2}<B<1 \\
10^{-1}<B<10^{-1 / 2} \\
10^{-2}<B<10^{-1} \\
B<10^{-2}\end{array}$ & $\begin{array}{c}55.2 \% \\
32.8 \% \\
6.8 \% \\
4.2 \% \\
1 \%\end{array}$ & $\begin{array}{c}66.4 \% \\
33.6 \% \\
0 \% \\
0 \% \\
0 \%\end{array}$ \\
\hline 30 & $\begin{array}{c}B>1 \\
10^{-1 / 2}<B<1 \\
10^{-1}<B<10^{-1 / 2} \\
10^{-2}<B<10^{-1} \\
B<10^{-2}\end{array}$ & $\begin{array}{c}60.6 \% \\
28.4 \% \\
7.8 \% \\
3.2 \% \\
0 \%\end{array}$ & $\begin{array}{c}70.2 \% \\
29.8 \% \\
0 \% \\
0 \% \\
0 \%\end{array}$ \\
\hline 50 & $\begin{array}{c}B>1 \\
10^{-1 / 2}<B<1 \\
10^{-1}<B<10^{-1 / 2} \\
10^{-2}<B<10^{-1} \\
B<10^{-2}\end{array}$ & $\begin{array}{c}59.8 \% \\
29.8 \% \\
8.2 \% \\
2 \% \\
0.2 \%\end{array}$ & $\begin{array}{c}69.4 \% \\
30.6 \% \\
0 \% \\
0 \% \\
0 \%\end{array}$ \\
\hline
\end{tabular}

Tabela 6.1: Valor de A e de $A_{v}$ em porcentagem por intervalo com $\beta_{1}=\beta_{2}$. 
Para melhor visualização dos dados da Tabela 6.1, vamos construir os gráficos relativos a cada tamanho de amostra, ou seja: para $n_{1}=n_{2}=10$ temos a Figura 6.1; para $n_{1}=n_{2}=30$, a Figura 6.2 e para $n_{1}=n_{2}=50$, a Figura 6.3.

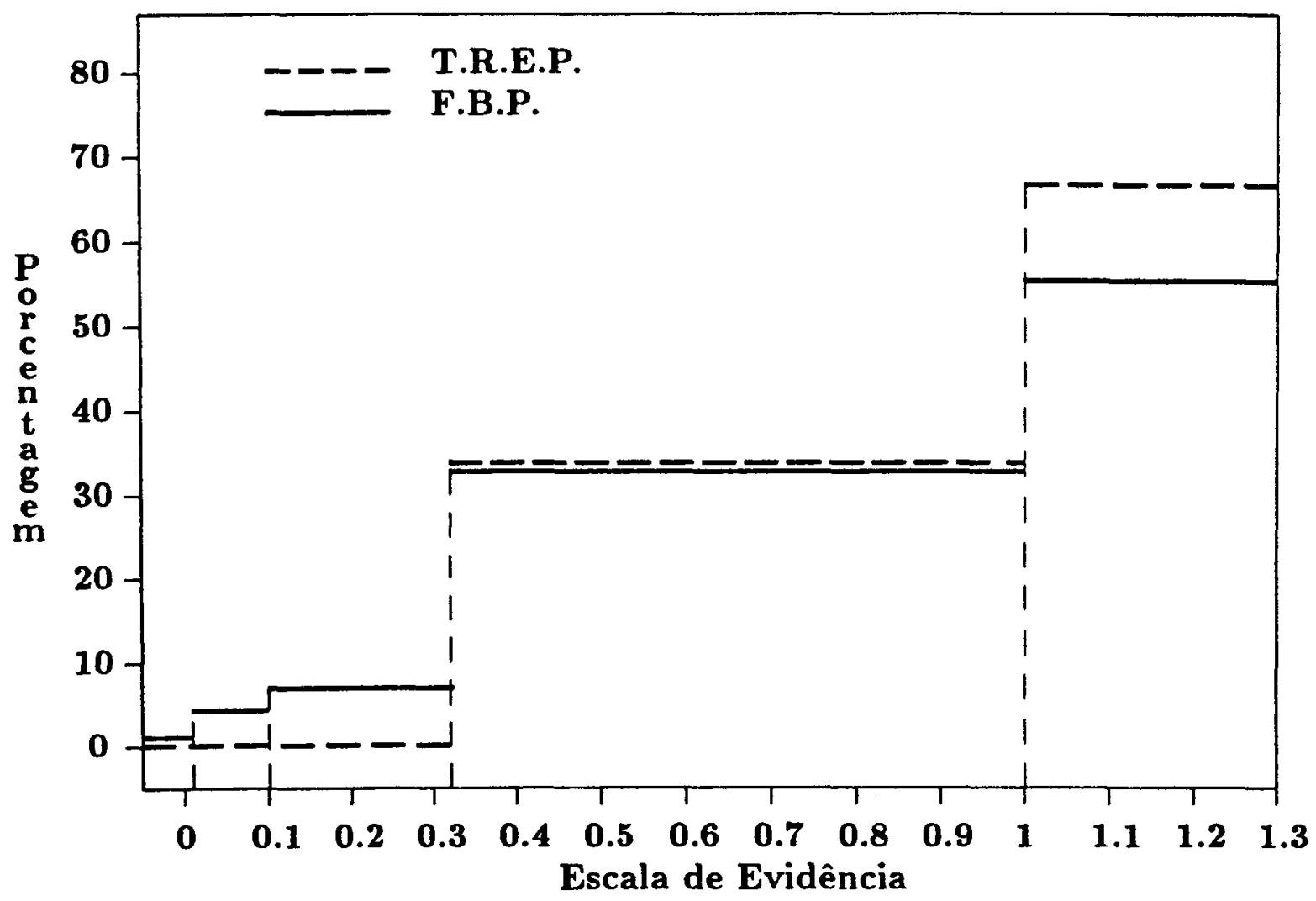

Figura 6.1: Gráfico da escala de evidência versus porcentagem para $n_{1}=n_{2}=10$ $\operatorname{com} \beta_{1}=\beta_{2}$. 


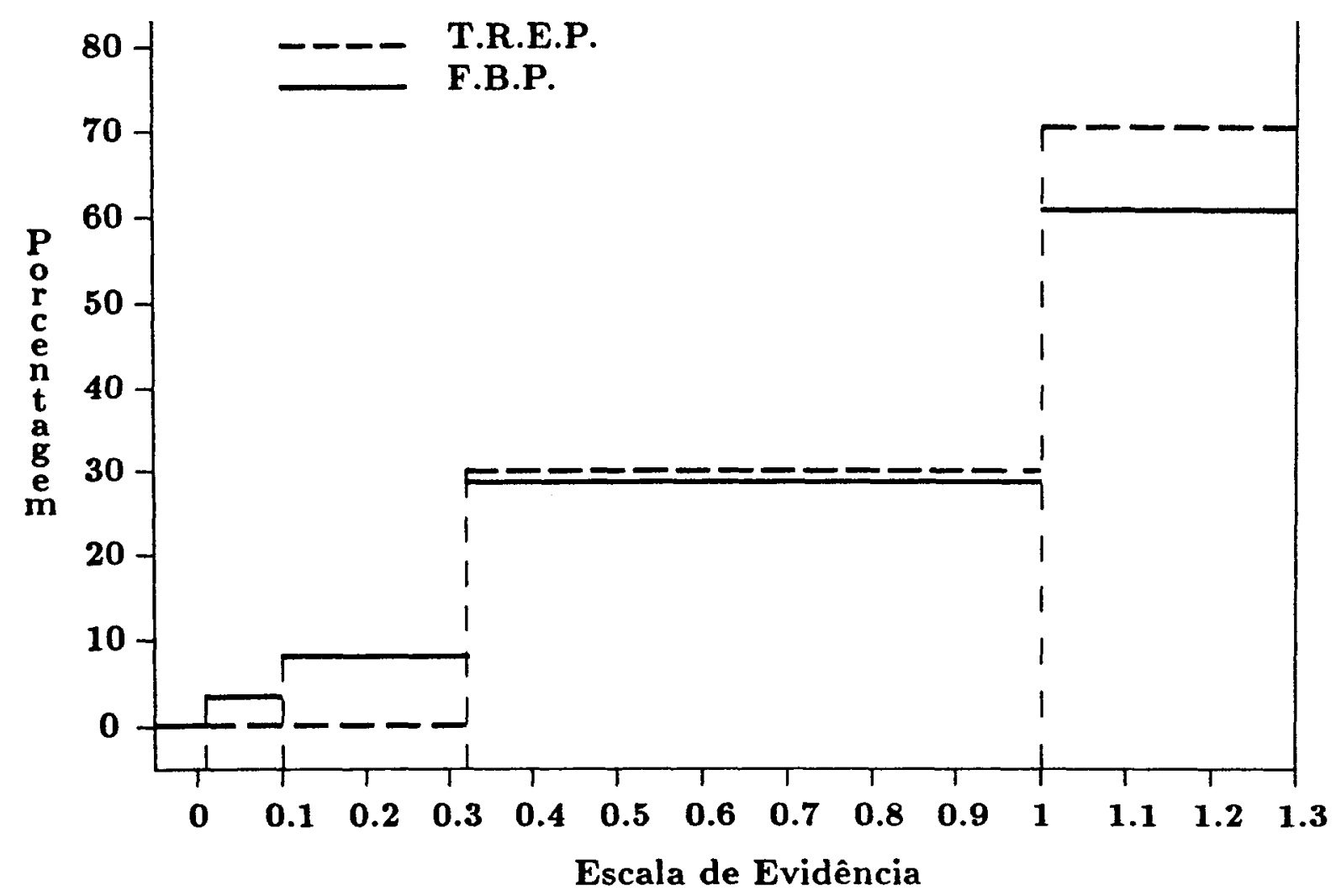

Figura 6.2: Gráfico da escala de evidência versus porcentagem para $n_{1}=n_{2}=30$ $\operatorname{com} \beta_{1}=\beta_{2}$. 


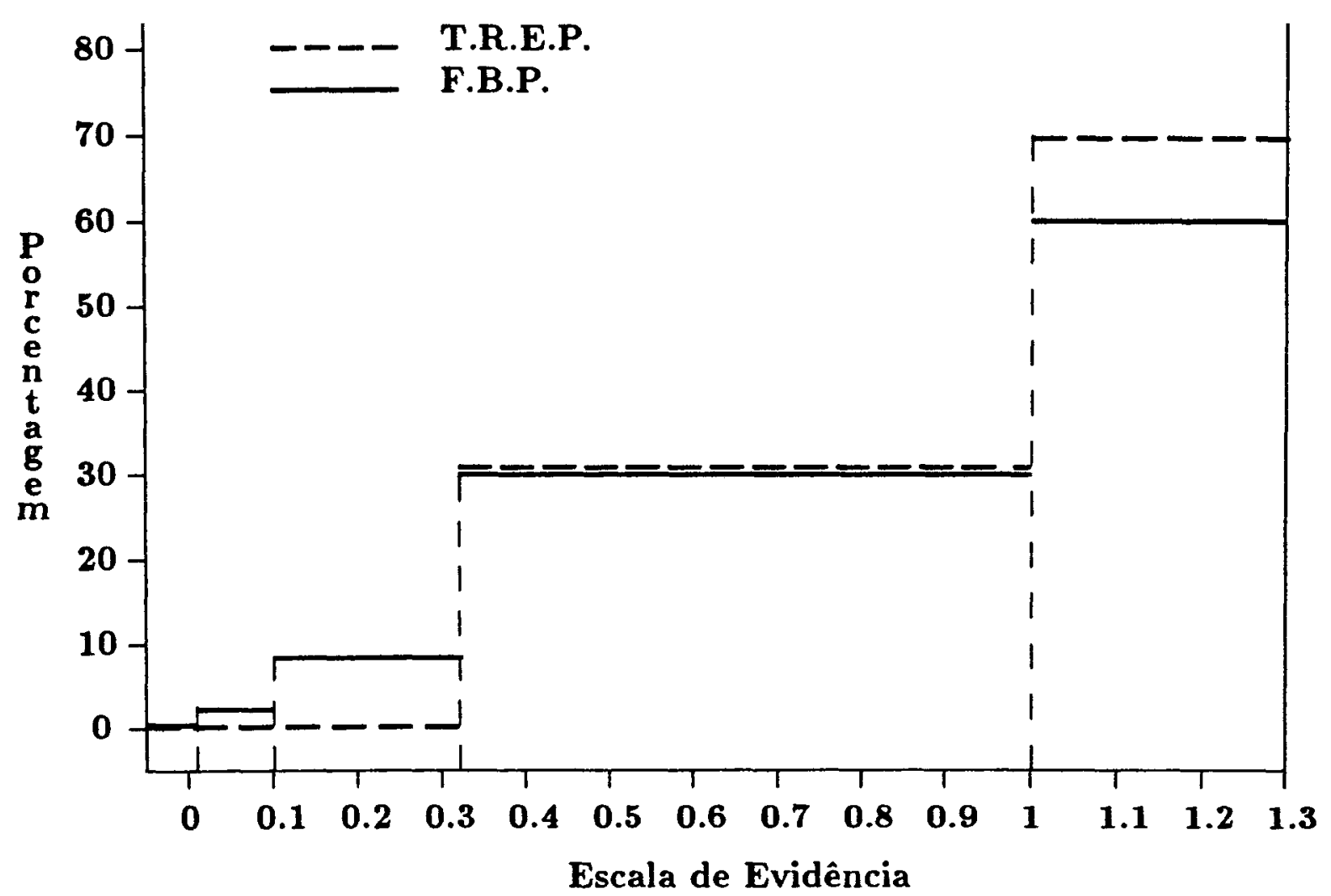

Figura 6.3: Gráfico da escala de evidência versus porcentagem para $n_{1}=n_{2}=50$ $\operatorname{com} \beta_{1}=\beta_{2}$. 
Verificamos nos três casos, Figuras 6.1, 6.2 e 6.3, que o teste da razão de entropia a posteriori supera o fator de Bayes a posteriori, isto porque ele se manteve nos dois primeiros intervalos da escala de evidência, que são os de apoiar o modelo $M_{1}$, e de fraca evidência, contra o modelo $M_{1}$, sugerindo uma forte evidência a favor do modelo verdadeiro. Além disso, ainda superou, em porcentagem, o fator de Bayes a posteriori nesses dois intervalos. Enquanto que o fator de Bayes a posteriori manteve-se em todos os intervalos, sendo que para os três outros intervalos em pequena porcentagem.

Com base nas conclusões anteriores, fizemos outra simulação considerando o caso em que os betas são diferentes, ou seja, $\beta_{1}=1$ e $\beta_{2}=3$, a fim de verificar se o teste da razão de entropia a posteriori é capaz de detectar com bastante conviç̧ão esta diferença. Repetimos o mesmo procedimento do caso anterior, estando os resultados obtidos na Tabela 6.2.

Tal como no caso anterior, representaremos os dados obtidos em gráficos. A Figura 6.4 representa a amostra de tamanho 10 e a Figura 6.5 a de tamanhos 30 e 50. 


\begin{tabular}{|c|c|c|c|}
\hline$n_{1}=n_{2}$ & escala & $\mathbf{A}$ & $A_{v}$ \\
\hline 10 & $\begin{array}{c}B>1 \\
10^{-1 / 2}<B<1 \\
10^{-1}<B<10^{-1 / 2} \\
10^{-2}<B<10^{-1} \\
B<10^{-2}\end{array}$ & $\begin{array}{l}0 \% \\
0 \% \\
0 \% \\
0 \% \\
100 \%\end{array}$ & $\begin{array}{c}0 \% \\
0 \% \\
99.2 \% \\
0.8 \% \\
0 \%\end{array}$ \\
\hline 30 & $\begin{array}{c}B>1 \\
10^{-1 / 2}<B<1 \\
10^{-1}<B<10^{-1 / 2} \\
10^{-2}<B<10^{-1} \\
B<10^{-2}\end{array}$ & $\begin{array}{l}0 \% \\
0 \% \\
0 \% \\
0 \% \\
100 \%\end{array}$ & $\begin{array}{c}0 \% \\
0 \% \\
100 \% \\
0 \% \\
0 \%\end{array}$ \\
\hline 50 & $\begin{array}{c}B>1 \\
10^{-1 / 2}<B<1 \\
10^{-1}<B<10^{-1 / 2} \\
10^{-2}<B<10^{-1} \\
B<10^{-2}\end{array}$ & $\begin{array}{l}0 \% \\
0 \% \\
0 \% \\
0 \% \\
100 \%\end{array}$ & $\begin{array}{c}0 \% \\
0 \% \\
100 \% \\
0 \% \\
0 \%\end{array}$ \\
\hline
\end{tabular}

Tabela 6.2: Valor de A e de $A_{v}$ em porcentagem por intervalo com $\beta_{1} \neq \beta_{2}$ 82 


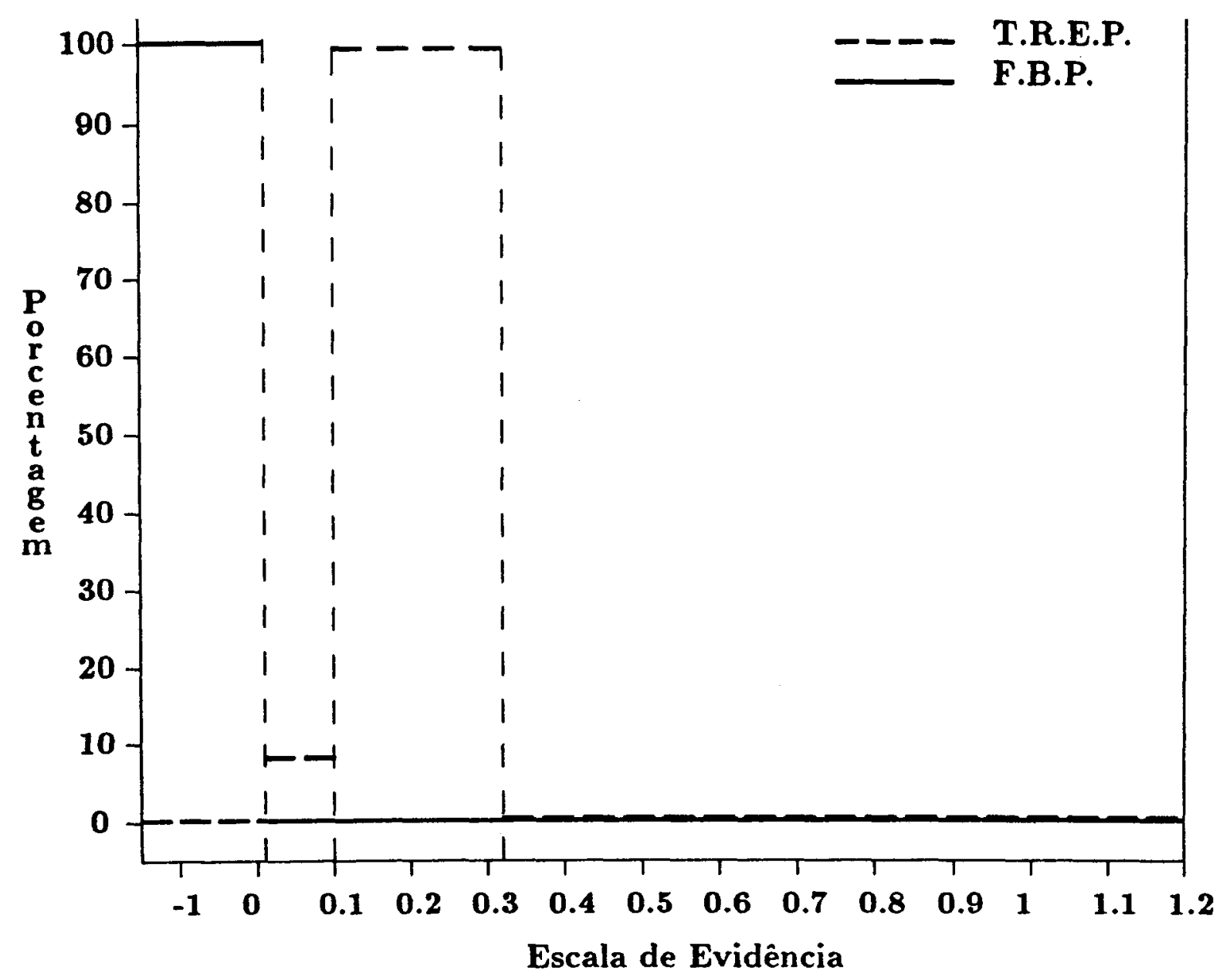

Figura 6.4: Gráfico da escala de evidência versus porcentagem para $n_{1}=n_{2}=10$ $\operatorname{com} \beta_{1} \neq \beta_{2}$. 


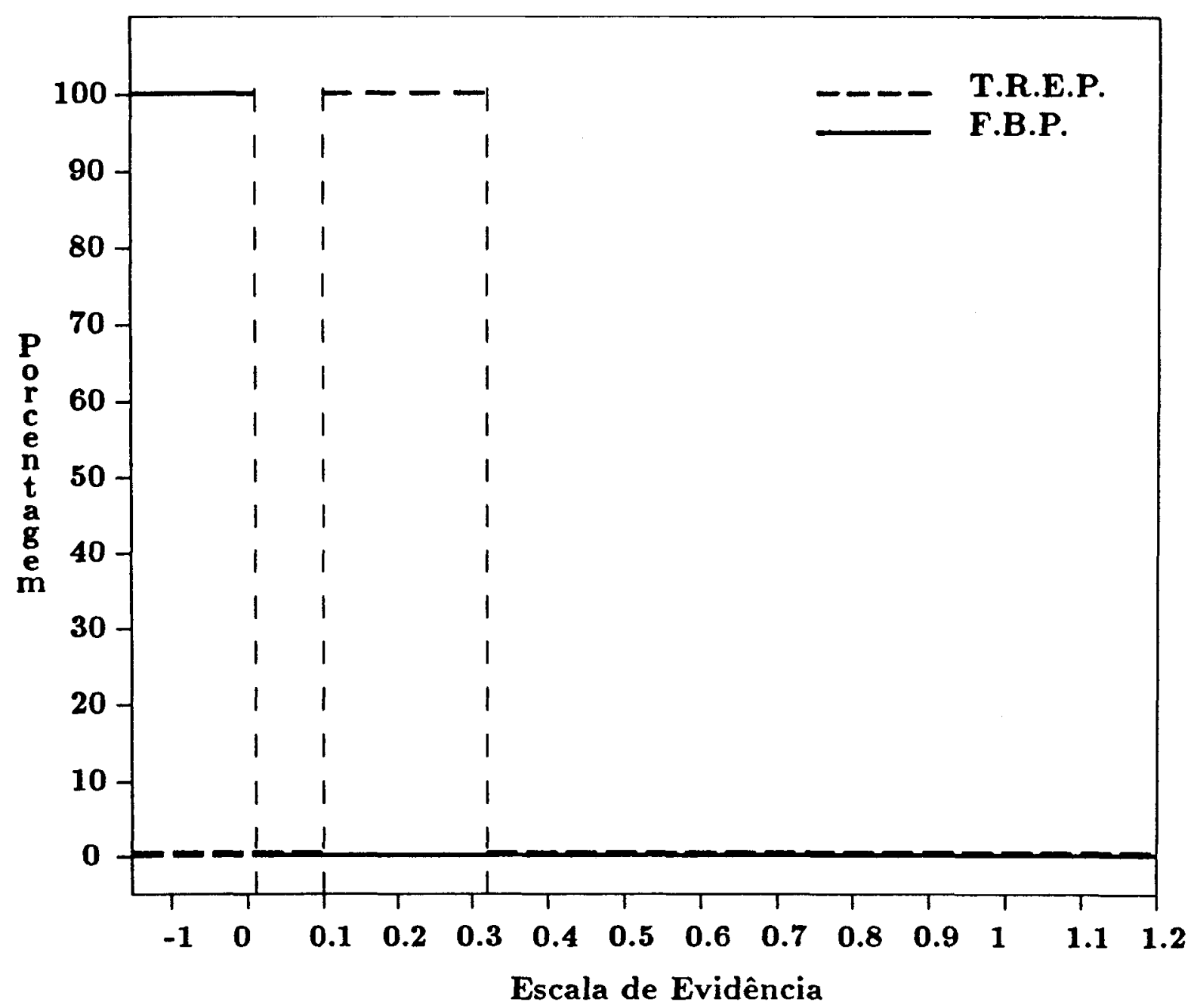

Figura 6.5: Gráfico da escala de evidência versus porcentagem para $n_{1}=n_{2}=30$ e 50 $\operatorname{com} \beta_{1} \neq \beta_{2}$. 
Como observamos nas Figuras 6.4 e 6.5 o teste da razão de entropia a posteriori não detectou fortemente a desigualdade dos betas, ou seja ele praticamente se manteve no intervalo de moderada evidência contra o modelo $M_{1}$. Entretanto, o fator de Bayes a posteriori manteve-se no intervalo decisivo contra o modelo $M_{1}$, ou seja, detectou com $100 \%$ de conviç̧ão o modelo $M_{2}$, superando o teste da razão de entropia a posteriori, quando o modelo alternativo é o verdadeiro.

Apesar do teste da razão de entropia a posteriori superar o fator de Bayes a posteriori quando o modelo $M_{1}\left(\beta_{1}=\beta_{2}\right)$ é o verdadeiro, nós propomos que quando a escala de evidência é a de Jeffreys, o melhor teste é o fator de Bayes a posteriori, pois ele detecta o modelo verdadeiro nas duas situações em que foram realizadas as simulações.

Como para a escala de Jeffreys não recomendamos o uso do teste da razão de entropia a posteriori, nós propomos o uso de outra escala de evidência para este teste, que seria a seguinte:

Escala de Evidência para o Teste da Razão de Entropia a Posteriori

$$
\begin{aligned}
& A_{v} \geq 1 \Longrightarrow \text { Forte evidência a favor de } M_{1} \\
& A_{v}<1 \Longrightarrow \text { Decisiva evidência contra } M_{1}
\end{aligned}
$$

Empregando a escala de evidência acima, reunimos resultados de simulações anteriores ( Tabelas 6.1 e 6.2 ) na Tabela 6.3, a seguir: 


\begin{tabular}{|c|c|c|c|}
\hline$n_{1}=n_{2}$ & escala & $\beta_{1}=\beta_{2}$ & $\beta_{1} \neq \beta_{2}$ \\
\hline \hline 10 & $A_{v} \geq 1$ & $66.4 \%$ & $0 \%$ \\
& $A_{v}<1$ & $33.6 \%$ & $100 \%$ \\
\hline 30 & $A_{v} \geq 1$ & $70.2 \%$ & $0 \%$ \\
\hline 50 & $A_{v}<1$ & $29.8 \%$ & $100 \%$ \\
\hline
\end{tabular}

Tabela 6.3: Valor de $A_{v}$ em porcentagem por intervalo

Através da Tabela 6.3 verificamos que o teste da razão de entropia a posteriori detecta tanto o modelo $M_{1}$ como o modelo $M_{2}$. Assim para esta escala, o teste da razāo de entropia a posteriori é muito bom.

Concluímos então, que o uso do fator de Bayes a posteriori ( A ) ou teste da razão de entropia a posteriori ( $\left.A_{v}\right)$, depende da escala utilizada. Quando emprega-se a escala de Jeffreys, a sugestão é usar o fator de Bayes a posteriori. Se a escala for $<1$ ou $\geq 1$, a sugestão é usar o teste da razão de entropia a posteriori. 


\section{Capítulo 7}

\section{Exemplo de Aplicação}

\subsection{Introdução}

A pós termos determinado, nos capítulos anteriores, uma metodologia Bayesiana para comparar os coeficientes de modelos de regressão, é de grande interesse, a título de ilustração, apresentarmos um exemplo prático de aplicação das técnicas obtidas. É o que faremos neste capítulo.

Os dados utilizados pertencem ao trabalho de pesquisa para tese de mestrado de Vieira (1992), e estão relacionados ao desenvolvimento de um sensor de corrente elétrica utilizando fibras ópticas como elemento sensor, cujo princípio de operação é o efeito Faraday, ou magneto-óptico, como também é conhecido. 


\subsection{Obtenção dos Dados}

Os dados foram obtidos através de um experimento onde o objetivo era determinar o comportamento da Constante de Verdet ( $V$ ) com a temperatura.

A determinação da Constante de Verdet ( $V$ ) é muito importante, pois com o conhecimento de todos os parâmetros que relacionam o ângulo de Faraday com a corrente elétrica, será possível realizar a calibração do sensor, que é o elemento que está sendo pesquisado.

A Constante de Verdet $(\mathrm{V})$ é obtida através da relação entre o ângulo de rotação de Faraday e a corrente elétrica, ou seja:

$$
F=V \cdot C \cdot n \cdot I
$$

onde

F : Ângulo de rotação de Faraday

V : Constante de Verdet

C : Fator de correção ou fator de forma

n : Número de espiras de condutor circulando a fibra

I : Corrente eletrica.

Os valores de $\mathrm{C}$ e $\mathrm{n}$ são constantes iguais a $\mathbf{0 . 9 9 8 1}$ e 4700 , respectivamente. 
Podemos observar que a Constante de Verdet ( V ) é obtida à partir da inclinação da reta ( F X I ) através de um ajuste de mínimos quadrados, ou seja, é o coeficiente angular da reta.

Assim sendo, podemos considerar o modelo definido na seção (2.2), e teremos a seguinte equivalência:

$$
\begin{aligned}
F & \Longleftrightarrow Y_{i j} \\
V \cdot C \cdot n & \Longleftrightarrow \beta_{j} \\
I & \Longleftrightarrow X_{i j},
\end{aligned}
$$

onde o interesse é verificar se $\beta_{j}$ são iguais ou diferentes, quando há mudança na temperatura.

Na Tabela 7.1 temos $\mathrm{Y}$ (ângulo de rotação - grau) e X (corrente elétrica - ampéres) para duas temperaturas: 30 e $\mathbf{4 0}$ graus centígrados.

\subsection{Verificação da Adequação do Modelo}

Vimos na seção (2.2) que, associado ao modelo de regressão linear simples, tínhamos um conjunto de suposições que eram admitidas válidas e que deverão ser verificadas neste exemplo.

Para verificarmos essas suposições, vamos fazer uma análise de resíduos que é uma etapa essencial de qualquer modelo de regressão pois, ana- 


\begin{tabular}{|c|c|c|c|c|}
\hline $\mathbf{j}$ & \multicolumn{2}{|c|}{$30^{\circ} \mathrm{C}$} & \multicolumn{2}{|c|}{$40^{\circ} \mathrm{C}$} \\
\hline i & $\mathbf{X}$ & $\mathbf{Y}$ & $\mathbf{X}$ & $\mathbf{Y}$ \\
\hline 1 & 0.000 & 0.00 & 0.000 & 0.00 \\
\hline 2 & 0.501 & 0.60 & 0.500 & 0.57 \\
\hline 3 & 1.007 & 1.25 & 1.003 & 1.30 \\
\hline 4 & 1.502 & 1.85 & 1.500 & 1.77 \\
\hline 5 & 2.008 & 2.50 & 1.997 & 2.55 \\
\hline 6 & 2.503 & 3.08 & 2.503 & 3.07 \\
\hline 7 & 3.000 & 3.73 & $\mathbf{3 . 0 0 3}$ & 3.61 \\
\hline 8 & 3.505 & 4.38 & 3.502 & 4.38 \\
\hline 9 & 4.004 & 4.92 & 4.001 & 4.93 \\
\hline 10 & 4.507 & 5.53 & 4.500 & $\mathbf{5 . 5 7}$ \\
\hline 11 & 5.006 & 6.11 & 5.000 & 6.28 \\
\hline 12 & 5.508 & 6.87 & $\mathbf{5 . 5 0 0}$ & 6.65 \\
\hline 13 & 6.005 & 7.42 & 6.000 & 7.50 \\
\hline 14 & 6.504 & 7.98 & 6.500 & 7.82 \\
\hline 15 & 7.000 & 8.58 & 7.000 & 8.50 \\
\hline
\end{tabular}

Tabela 7.1: Os dados obtidos considerando duas temperaturas

lizando os resíduos, podemos ter informações sobre a validade ou possíveis violações das suposições do modelo adotado. Utilizamos um procedimento gráfico para verificar a adequabilidade do modelo. 
Nas Figuras 7.1 e 7.2 apresentamos o gráfico do resíduo versus a variável independente, através do qual podemos verificar se a função de regressão linear é apropriada e ainda examinar se a variância do erro é constante.

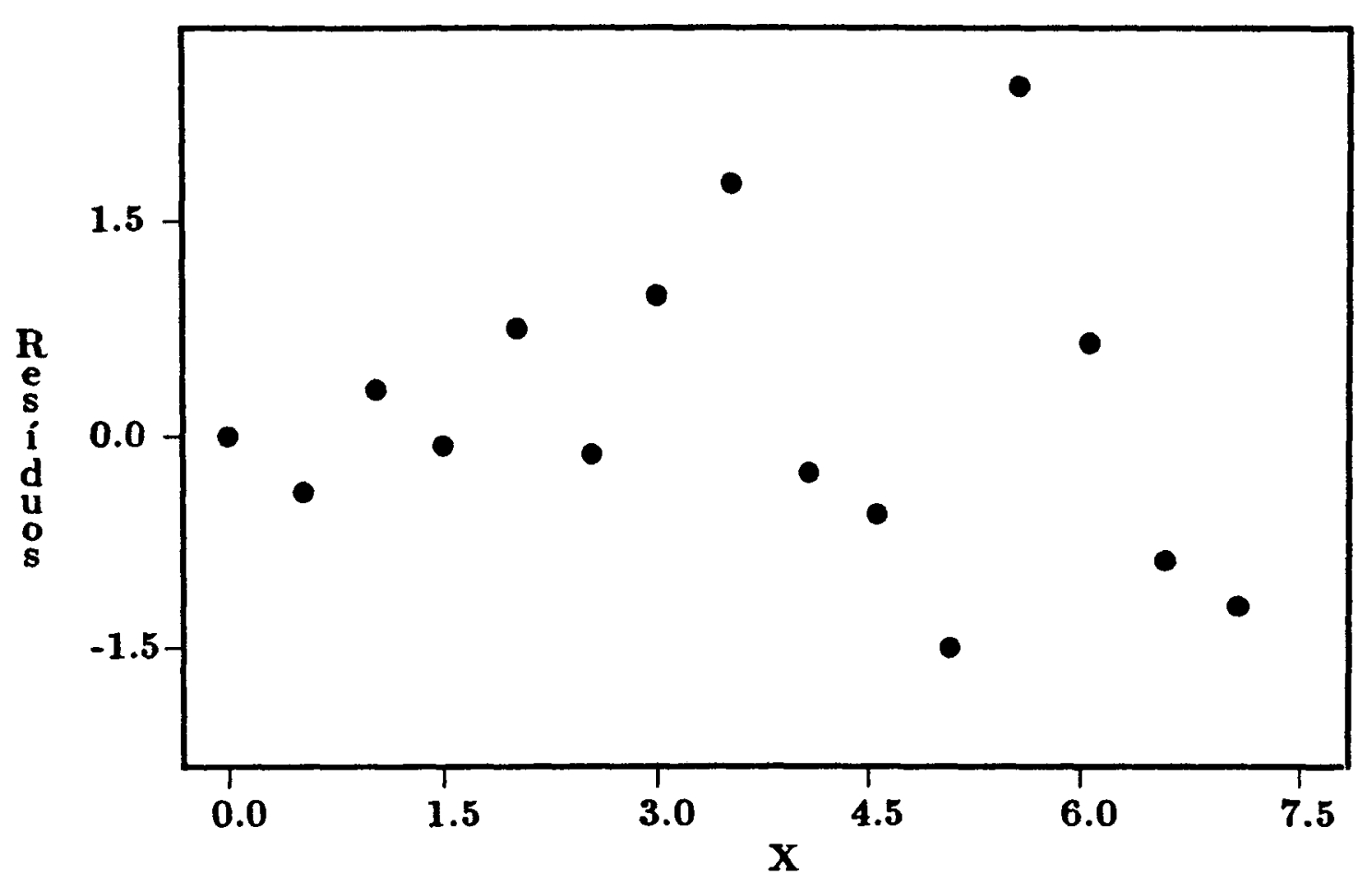

Figura 7.1: Gráfico do resíduos versus variável independente $\mathrm{X}$ para a temperatura de $30^{\circ} \mathrm{C}$. 


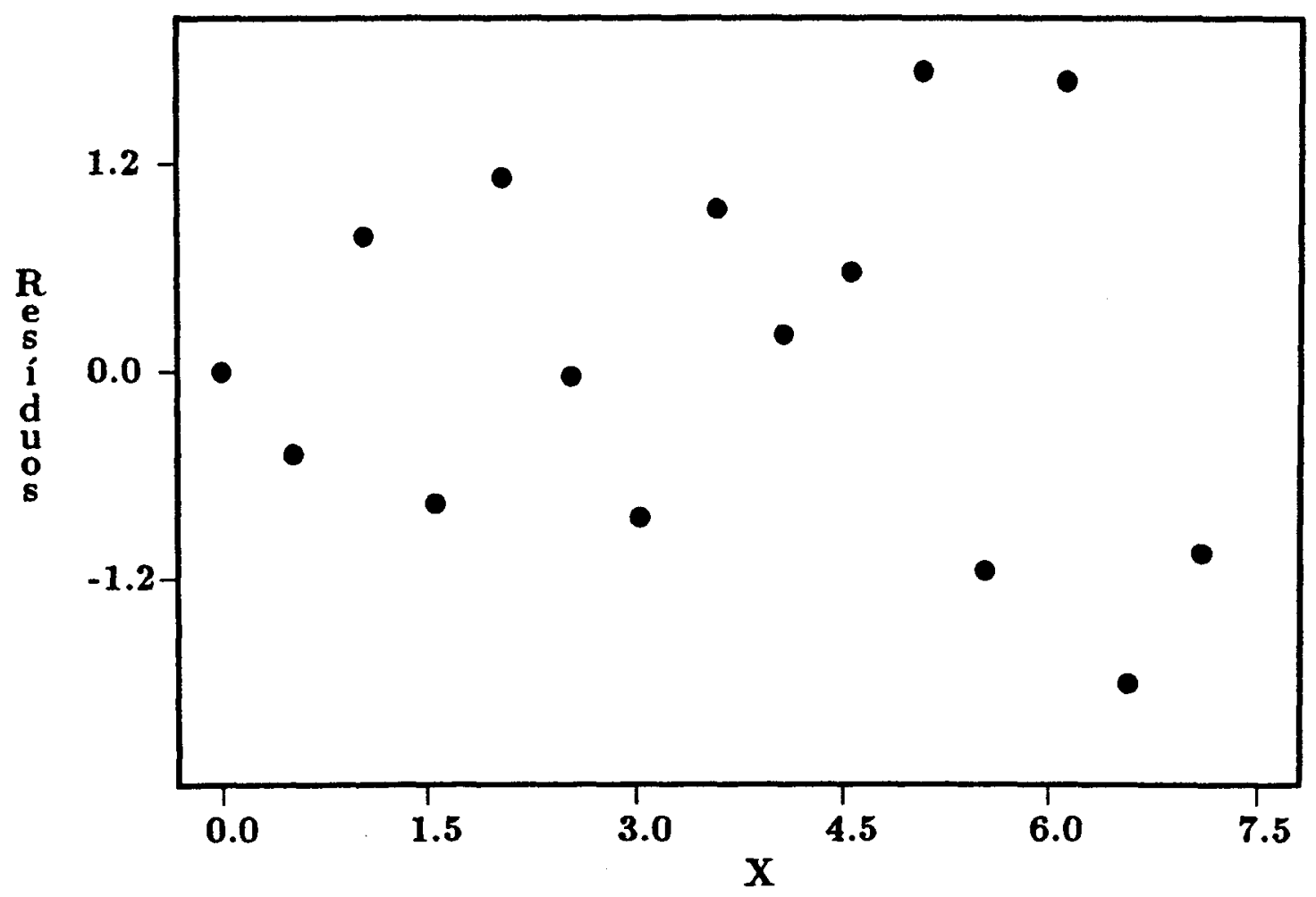

Figura 7.2: Gráfico do resíduos versus variável independente $\mathrm{X}$ para a temperatura de $40^{\circ} \mathrm{C}$.

As Figuras 7.1 e 7.2 sugerem que o erro da variância cresce com $X$, ou seja, um problema de heterocedasticidade, indicando que não vale a suposição $\operatorname{Var}\left(e_{i}\right)=\sigma^{2}$. Mas para nos certificarmos da existência de heterocedasticidade, fizemos o teste de Glejser (1969), onde encontramos para a temperatura de $30^{\circ} \mathrm{C}$ o valor observado da estatítica $\mathrm{F}$ como sendo $7.96>F(1,13,0.95)=4.67$ e para a temperatura de $40^{\circ} C$, o valor observado 
da estatítica $F$ como sendo $10.25>F(1,13,0.95)=4.67$, confirmando o que visualizamos nos gráficos.

Para eliminar este problema transformamos os dados, ou seja, dividimos por $\sqrt{X}$ as variáveis. Assim as novas variáveis serão:

$$
X^{\prime}=\frac{X}{\sqrt{X}}=\sqrt{X} \quad \text { e } \quad Y^{\prime}=\frac{Y}{\sqrt{X}} .
$$

Os dados transformados são apresentados na Tabela 7.2.

\begin{tabular}{|c|c|c|c|c|}
\hline $\mathbf{j}$ & \multicolumn{2}{|c|}{$30^{\circ} \mathrm{C}$} & \multicolumn{2}{|c|}{$40^{\circ} \mathrm{C}$} \\
\hline i & $X^{\prime}$ & $Y^{\prime}$ & $X^{\prime}$ & $Y^{\prime}$ \\
\hline 1 & 0.00000 & 0.00000 & 0.00000 & 0.00000 \\
\hline 2 & 0.70781 & 0.84768 & 0.70711 & 0.80610 \\
\hline 3 & 1.00349 & 1.24565 & 1.00150 & 1.29805 \\
\hline 4 & 1.22556 & 1.50951 & 1.22474 & 1.44520 \\
\hline 5 & 1.41704 & 1.76424 & 1.41315 & 1.80448 \\
\hline 6 & 1.58209 & 1.94680 & 1.58209 & 1.94047 \\
\hline 7 & 1.73205 & 2.15352 & 1.73292 & 2.08319 \\
\hline 8 & 1.87216 & 2.33954 & 1.87136 & 2.34054 \\
\hline 9 & 2.00100 & 2.45877 & 2.00025 & 2.46469 \\
\hline 10 & 2.12297 & 2.60484 & 2.12132 & 2.62572 \\
\hline 11 & 2.23741 & 2.73084 & 2.23607 & 2.80850 \\
\hline 12 & 2.34691 & 2.92725 & 2.34521 & 2.83557 \\
\hline 13 & 2.45051 & $\mathbf{3 . 0 2 7 9 4}$ & 2.44949 & 3.06186 \\
\hline 14 & 2.55029 & $\mathbf{3 . 1 2 9 0 5}$ & 2.54951 & 3.06726 \\
\hline 15 & 2.64575 & 3.24294 & 2.64575 & 3.21270 \\
\hline
\end{tabular}

Tabela 7.2: Os dados transformados de duas temperaturas 
Os gráficos, resíduos versus a variável independente $\left(X^{\prime}\right)$ estão representados nas Figuras 7.3 e 7.4 onde observamos que a variância dos erros é constante. Também fizemos o teste de Glejser (1969) e obtivemos os resultados $\mathbf{F}=2.64<4.67$ e $\mathbf{F}=0.47<4.67$ para as temperatura de $30^{\circ} \mathrm{C}$ e $40^{\circ} \mathrm{C}$, respectivamente, confirmando assim a não existência de heterocedasticidade.

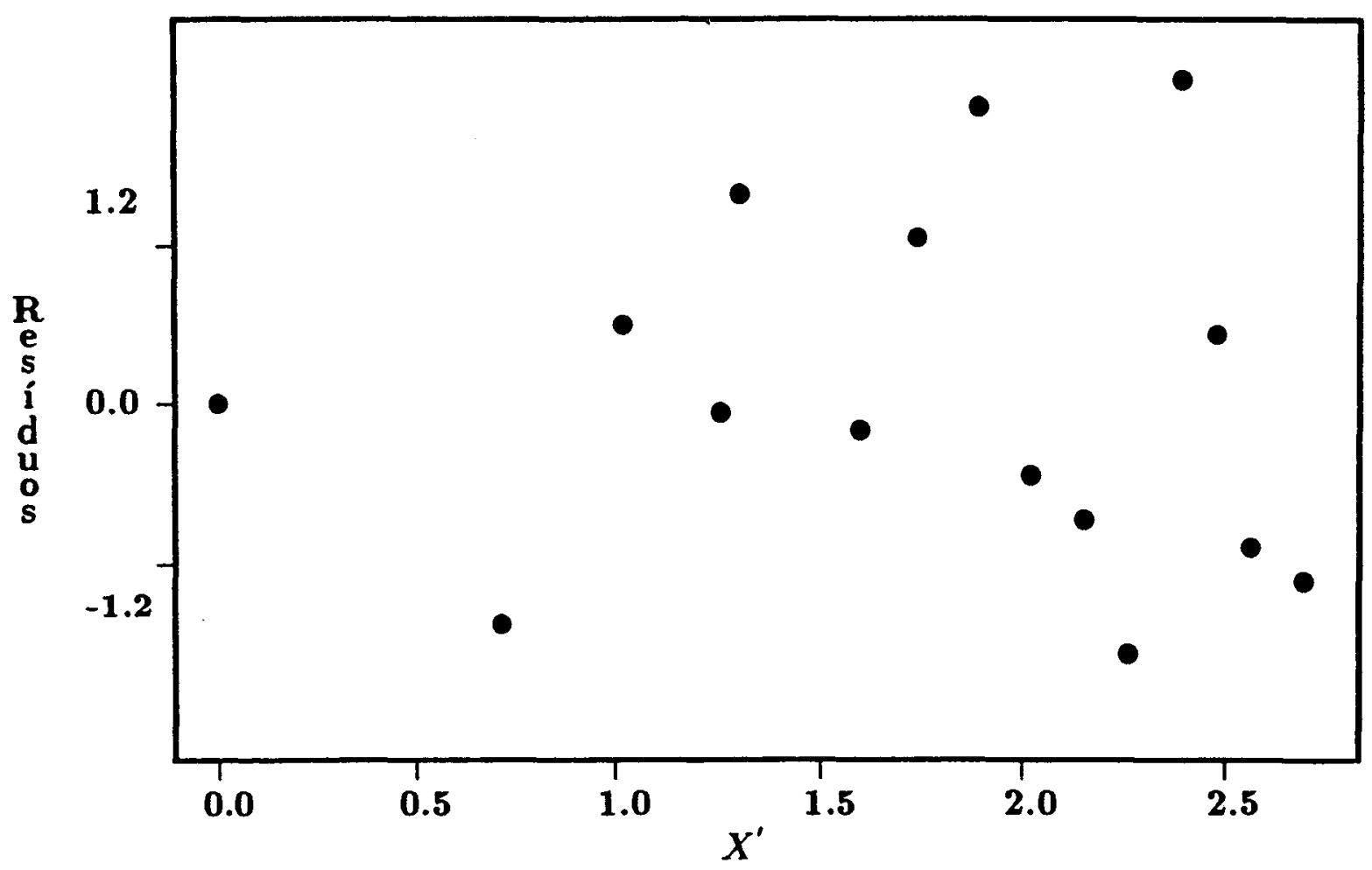

Figura 7.3: Gráfico do resíduos versus variável independente $X^{\prime}$ para a temperatura de $30^{\circ} \mathrm{C}$. 


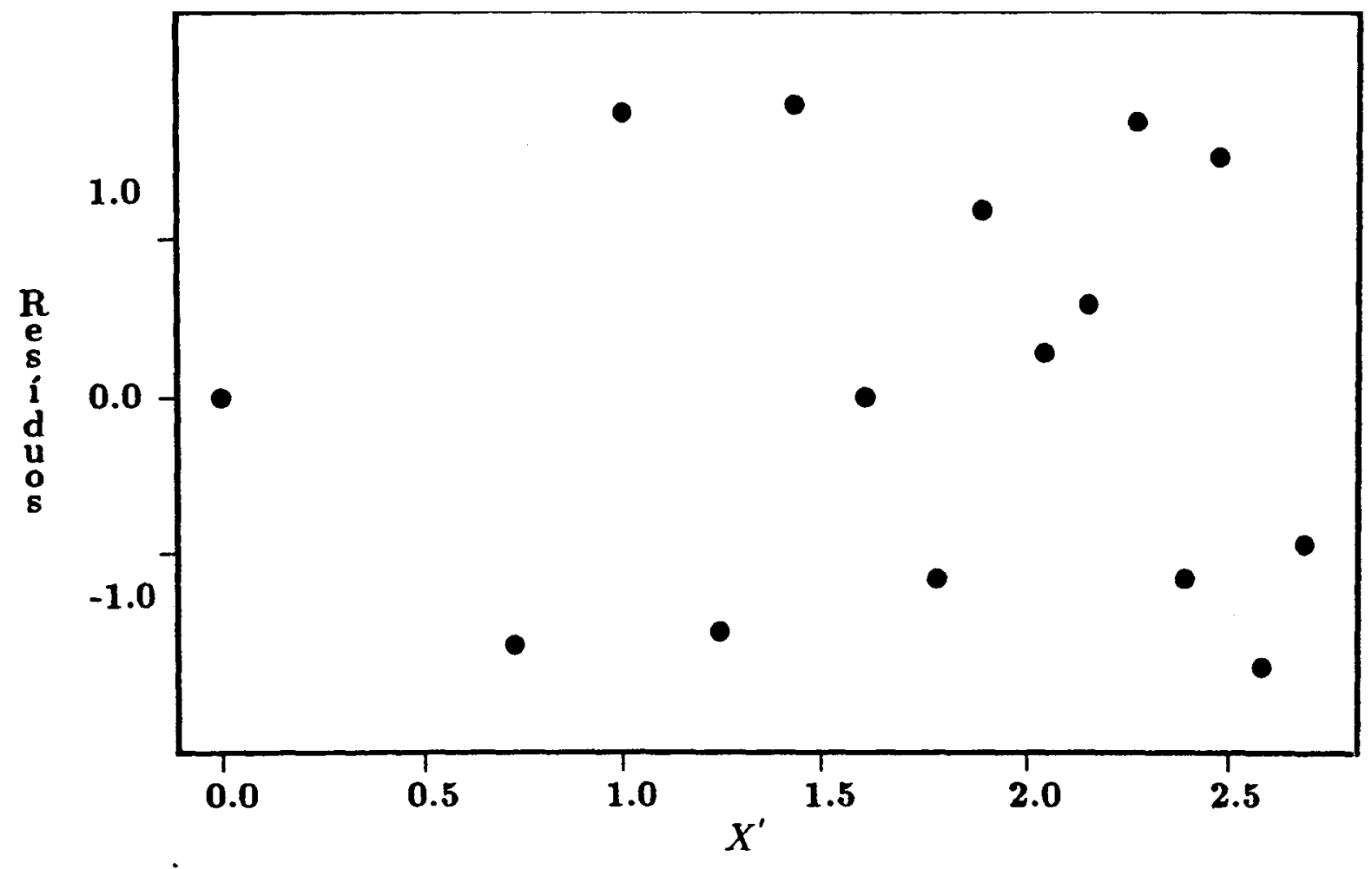

Figura 7.4: Gráfico do resíduos versus variável independente $X^{\prime}$ para a temperatura de $40^{\circ} \mathrm{C}$.

Apesar de não apresentarmos o gráfico de Nscor x Resíduos, eles foram feitos e observamos uma normalidade razoável. Verificamos também a existência de autocorrelação dos resíduos através do teste de Durbin-Watson, onde encontramos para a temperatura de $30^{\circ} \mathrm{C}(\mathrm{d}=1.98)$ e para a temperatura de $40^{\circ} \mathrm{C}(\mathrm{d}=3.03)$. Em ambos os casos, detectou-se que $e_{i j} e e_{r s}$ são não correlacionados para todo $(i, j) \neq(r, s)$. Assim sendo, podemos considerar 
que o modelo é adequado.

\subsection{Teste para Comparar os Coeficientes}

Antes de comparar os coeficientes, precisamos testar as variâncias, ou seja $H_{0}: \sigma_{1}^{2}=\sigma_{2}^{2}$ e $H_{1}: \sigma_{1}^{2} \neq \sigma_{2}^{2}$. Usamos para isto o teste de Bartlett, o qual é ilustrado na Tabela 7.3.

\begin{tabular}{|c|ccccc|}
\hline $\mathbf{j}$ & $S_{j}^{2}$ & $d f_{j}$ & $\left(d f_{j}\right) S_{j}^{2}$ & $\ln S_{j}^{2}$ & $\left(d f_{j}\right) \ln S_{j}^{2}$ \\
\hline $30^{\circ} C$ & $\mathbf{0 . 0 0 0 3 6 3}$ & 14 & $\mathbf{0 . 0 0 5 0 8 4 8}$ & $\mathbf{- 7 . 9 2 0 6}$ & $-\mathbf{1 1 0 . 8 8 8 4}$ \\
$40^{\circ} C$ & $\mathbf{0 . 0 0 2 5 1 6}$ & 14 & $\mathbf{0 . 0 3 5 2 2 4}$ & $\mathbf{- 5 . 9 8 5 1}$ & $-\mathbf{8 3 . 7 9 1 4 8}$ \\
\hline
\end{tabular}

Tabela 7.3: Elementos para calcular o teste de Bartlett

Usando os valores da Tabela 7.3 , calculamos:

$$
\begin{gathered}
M S E=0.0014392 ; \ln M S E=-6.5436 ; \quad C=1.0357 e \\
B=11.064>\chi_{(0.95,1)}^{2}=3.84 .
\end{gathered}
$$

Comparando o valor crítico do teste qui-quadrado com o obtido para $B$, podemos concluir que as variâncias são diferentes.

Como o teste indicou que as variâncias são diferentes, ficaria complicado comparar os coeficientes usando o enfoque clássico. Entretanto esta 
comparação é facilmente realizada se utilizarmos o fator de Bayes a posteriori definido em (3.31).

Considerando os dados da Tabela 7.2 e utilizando as expressões (3.22), (3.23), (3.27), (3.26) e (3.25) encontramos, respectivamente,

$\begin{array}{rlrlrl}\hat{\beta}_{1} & =1.23288 & & \text { e } & \hat{\beta}_{2} & =1.22836 ; \\ R S S_{1} & =0.0050838 & & \text { e } & R S S_{2} & =0.0352192 ; \\ a_{1} & =155082 & & \text { e } & a_{2} & =22363.8 ; \\ \hat{\beta} & =1.23231 & \text { e } & \text { S } & =0.398822\end{array}$

Calculando o fator de Bayes a posteriori para $r=1$, que é dado por $A=1.15854$, detectou-se com bastante convicção a igualdade dos betas. Com este resultado, podemos concluir que a Constante de Verdet ( V ) não se altera com a temperatura, coincidindo com os resultados obtidos por Vieira (1992). 


\section{Capítulo 8}

\section{Conclusões Finais e Perspectivas Futuras}

Em todo o desenvolvimento desta dissertação, assumimos modelos de regressão linear e testes para comparar os coeficientes desses modelos, com densidades a priori não informativas. Explorando o uso do método de Laplace para aproximação de integrais, conseguimos as soluções analíticas para as densidades a posteriori de interesse. Nestas condições várias conclusões foram obtidas e são sumarizadas a seguir.

Para todas as situações consideradas nos Capítulos 3 e 4, obtivemos resultados satisfatórios ao nosso propósito, ou seja, confirmaram a insensibilidade em relação a priori. Também, quando aumentamos o tamanho da amostra, o fator de Bayes a posteriori permaneceu estável, não ocorrendo portanto o paradoxo de Lindley.

É importante lembrar que os resultados para o fator de Bayes a 
posteriori em (4.16), obtidos através do modelo condicional introduzido por Rodrigues e Cordani (1990), mostraram-se coincidentes com os resultados obtidos em (3.99) por Bolfarine, Rodrigues e Cordani (1992).

Observamos também que o teste no enfoque Bayesiano (fator de Bayes a posteriori) mostrou-se superior quando comparado ao teste no enfoque clássico (teste da razão de verossimilhança), ver Capítulo 5. Entretanto, quando comparamos com outro teste no enfoque Bayesiano (teste da razão de entropia a posteriori) ele não foi tão bom, pois dependendo da escala adotada, o teste da razão de entropia a posteriori é melhor.

No exemplo prático apresentado, não foi possível fazer uma análise clássica devido ser extremamente complicado quando se trata de variâncias diferentes. No entanto, a comparação através do fator de Bayes a posteriori foi realizada com razoável facilidade.

Finalmente, concluímos que, o uso dos métodos Bayesianos para comparação de modelos pode ser de grande interesse devido a sua simplicidade e precisão.

\subsection{Perspectivas Futuras}

Como continuação deste projeto de tese, pretendemos futuramente pesquisar outros tópicos, tais como:

1. Comparar o fator de Bayes a posteriori com o teste da razão de entropia 
a posteriori para distribuições não normais, como por exemplo a distribuição exponencial,

$$
X_{i j} \sim E\left(\theta_{j}\right), \quad \begin{aligned}
i & =1, \ldots, n_{j} \\
\mathbf{j} & =1,2,
\end{aligned}
$$

onde o objetivo será testar a hipótese $H_{0}: \theta_{1}=\theta_{2}=\theta$ contra a hipótese alternativa $H_{1}: \theta_{1} \neq \theta_{2}$. O modelo sob $H_{0}$ chamariamos de $M_{1}$ e o modelo sob $H_{1}$ chamaríamos de $M_{2}$.

Para o modelo $M_{1}$ a função de verossimilhança é dada por:

$$
L(\theta)=\frac{1}{\theta^{n_{1}+n_{2}}} \exp \left\{-\frac{1}{\theta} \sum_{j=1}^{2} \sum_{i=1}^{n_{j}} X_{i j}\right\} .
$$

Sob o modelo $M_{2}$ a função de verossimilhança é dada por:

$$
L\left(\theta_{1}, \theta_{2}\right)=\prod_{j=1}^{2} \frac{1}{\theta_{j}^{n_{j}}} \exp \left\{-\frac{1}{\theta_{j}} \sum_{i=1}^{n_{j}} X_{i j}\right\}
$$

Considerando as expressões acima pretendemos verificar a possibilidade de obter o fator de Bayes a posteriori através dos resultados obtidos por Rodrigues, Bolfarine e Louzada-Neto (1993). Também, pretendemos obter o teste da razão de entropia a posteriori para este modelo e comparar com o fator de Bayes a posteriori através de simulações.

2. Comparar o teste da razão de entropia a posteriori com o Fator de Bayes Intrínseco, que foi proposto recentemente por Berger e Pericchi (1993). 


\section{APÊNDICE 1 Fórmulas}

a) $\quad \int_{0}^{\infty} x^{-(p+1)} e^{-a / x} d x=a^{-p} \Gamma(p)$

para $a>0$ e $p>0$.

b) $\sum_{i=1}^{n}\left(Y_{i}-\beta_{0}-\beta_{1} X_{i}\right)^{2}=\nu S^{2}+n\left(\beta_{0}-\widehat{\beta}_{0}\right)^{2}+\left(\beta_{1}-\widehat{\beta}_{1}\right)^{2} \sum_{i=1}^{n} X_{i}^{2}+$ $+2\left(\beta_{0}-\hat{\beta}_{0}\right)\left(\beta_{1}-\hat{\beta}_{1}\right) \sum_{i=1}^{n} X_{i}$

onde

$$
\begin{gathered}
\nu=n-2, \\
\hat{\beta}_{0}=\bar{Y}-\beta_{0} \bar{X},
\end{gathered}
$$




$$
\begin{aligned}
\hat{\beta}_{1} & =\frac{\sum_{i=1}^{n}\left(Y_{i}-\bar{Y}\right)\left(X_{i}-\bar{X}\right)}{\sum_{i=1}^{n}\left(X_{i}-\bar{X}\right)^{2}} e \\
S^{2} & =\frac{\sum_{i=1}^{n}\left(Y_{i}-\hat{\beta}_{0}-\hat{\beta}_{1} X_{i}\right)^{2}}{n-2}
\end{aligned}
$$

c) $\ln (1+x) \doteq x \quad ;|x|<1$. 


\section{APÊNDICE 2 \\ Aproximação de Integrais pelo Método de Laplace}

A utilização de métodos Bayesianos usualmente exige cálculos (cálculos de integrais) envolvendo as densidades a posteriori e as densidades preditivas de interesse. Estes cálculos em geral requerem métodos de aproximações numéricas, pois as soluções analíticas dessas integrais, em geral não são possiveis.

Um método muito utilizado em inferência Bayesiana aproximada é o método de LAPLACE para aproximação de integrais (ver por exemplo, Tierney e Kadane, 1986). O método de LAPLACE é um simples e eficaz método de expansão assintótica de integrais, ou seja esta aproximação está baseada na distribuição normal (depois de desenvolver em serie de TAYLOR). Embora o ponto de vista seja Bayesiano os resultados são formais e tem aplicações para inferências nāo Bayesianas.

Supondo que $h(\theta)$ seja uma função unimodal, para aplicar o 
método, necessitamos maximizar a função $-h(\theta)$, isto é encontrar $\hat{\theta}$ que maximiza $-h(\theta)$.

Supor que temos interesse em resolver a integral em $\theta$, dada por,

$$
I=\int f(\theta) \exp [-n h(\theta)] d \theta \text {. }
$$

No caso uniparamétrico $(\theta \in R)$, se $\hat{\theta}$ é o valor que maximiza $-h(\theta)$ em $\left(\right.$ A.1) e $\sigma=h^{\prime \prime}(\hat{\theta})^{-1 / 2}$, então o método de aproximação de LAPLACE é dado por,

$$
\hat{I} \simeq(2 \pi / n)^{1 / 2} \sigma f(\hat{\theta}) \exp [-n h(\hat{\theta})]
$$

No caso multiparamétrico, $\operatorname{com} \theta \epsilon R^{m}$, a aproximação de LAPLACE para (A.1) é dada por

$\hat{I} \simeq(2 \pi)^{m / 2}\left[\operatorname{det}\left(n D^{2} h(\underset{\tilde{\theta}}{\sim})\right)\right]^{-1 / 2} f(\underset{\sim}{\tilde{\theta}}) \exp [-n h(\underset{\sim}{\tilde{\theta}})]$,

onde o vetor $\underset{\sim}{\hat{\theta}}$ maximiza a matriz $-h(\underset{\sim}{\theta})$ e $D^{2} h(\underset{\sim}{\hat{\theta}})$ é matriz Hessiana, de $h(\underset{\sim}{\theta})$ calculada em $\underset{\sim}{\tilde{\theta}}$.

Um tratamento minucioso da teoria de aproximações de integrais de LAPLACE acima considerado, pode ser encontrado em Kass, Tierney e Kadane (1990).

Um caso especial de aproximação de LAPLACE para integrais da 
forma,

$$
\int e^{-n h(\theta)} d \theta
$$

pode ser encontrado em Tierney e Kadane 1986 e 1989. 


\section{APÊNDICE 3 \\ Família da Verossiminhança Penalizada}

Com o objetivo de unificar os critérios para seleção de modelos existentes na literatura, Smith e Spiegelhalter (1980) propusseram uma classe de estatística definida genericamente da seguinte forma:

$$
\Lambda(m)=\lambda-m \nu,
$$

onde $\lambda=-2 \ln L_{1}\left(\hat{\Theta}_{1}\right) / L_{2}\left(\hat{\Theta}_{2}\right)$ e $\nu=p_{2}-p_{1}$. O $\hat{\Theta}_{j}$ é o estimador de maxima verossimilhança e $p_{j}$ é o número de parâmetros do modelo $\mathrm{j}$ ( $\mathrm{j}=1$, 2 ).

Grande número de critérios sugeridos para seleção de modelo pode ser considerado como casos particulares desta família. O AIC (Akaike Information Criterion - 1973) corresponde a $m=2$ e é assintoticamente equivalente 
a um critério de validação cruzada proposto por Stone (1977). A versão do fator de Bayes local (Smith; Spiegelhalter - 1980) corresponde a $m=3 / 2$. O GLIM procedimento baseado no gráfico da deviance contra o grau de liberdade corresponde a $m=1$ (Nelder ; Wedderburn - 1974). A estatítica da razão de verossimilhança corresponde a $m=0$. Também o fator de Bayes global (Smith; Spiegelhalter - 1980) corresponde a $m=\log \left\{n a^{1 / p_{2}-p_{1}}\right\}$, uma generalização do critério proposto por Schwarz (1978). A grande vantagem de estar nesta família é poder comparar os seus elementos através de gráficos. 


\section{APÊNDICE 4 Parâmetros Ortogonais}

Seja $\theta=\left(\theta_{1}, \theta_{2}\right)$ onde $\theta_{1}$ é um escalar e $\theta_{2}$ é um vetor de dimensão p. Seja $l(\theta)$ o logaritmo da função de verossimilhança dos dados e

$$
\begin{aligned}
J(\theta) & =-\frac{\partial^{2} l(\theta)}{\partial \theta^{2}} \\
& =\left(\begin{array}{ll}
J_{11} & J_{12} \\
J_{21} & J_{22}
\end{array}\right) .
\end{aligned}
$$

onde $J_{i j}=-\partial l(\theta) / \partial^{2} \theta_{i} \theta_{j}$. Os parâmetros $\theta_{1}$ e $\theta_{2}$ são ortogonais se:

$$
I_{12}=E_{\theta}\left[J_{12}\right]=0
$$

para todo $\theta$. 
Segundo Cox e Reid (1987), a ortogonalidade é uma forma de controlar a influência do parâmetro $\theta_{2}$ na estimativa do parâmetro de interesse $\theta_{1}$. Quando $\theta_{1} e \theta_{2}$ não são ortogonais. Cox e Reid sugerem substituir $\theta_{2}$ por $\phi=f\left(\theta_{1}, \theta_{2}\right)$, onde $\phi$ é ortogonal a $\theta_{1}$. O parâmetro $\phi$ é obtido como uma solução do sistema de equações diferenciais

$$
I_{22} \frac{\partial \theta_{2}}{\partial \theta_{1}}=-I_{12}
$$

onde $\partial \theta_{2} / \partial \theta_{1}$ é o vetor das derivadas parciais dos componentes de $\theta_{2}$ em relação a $\theta_{1}$. Este resultado permite obter uma reparametrização de $\left(\theta_{1}, \theta_{2}\right)$ para $\left(\theta_{1}, \phi\right)$, onde $\theta_{1} e \phi$ são ortogonais. A matriz $I(\theta)=E_{\theta}[J(\theta)]$ é conhecida como a "matriz de informação de Fisher" . 


\section{APÊNDICE 5 Tabelas das Constantes $\left(C_{r} 2^{1 / 2}\right)$}

\begin{tabular}{|c|ccc|}
\hline \multirow{2}{*}{$n_{1}=n_{2}$} & \multicolumn{3}{|c|}{$C_{r} 2^{1 / 2}$} \\
\cline { 2 - 4 } 10 & $1 / 2$ & 1 & $3 / 2$ \\
\hline \hline 30 & 1.4514 & 1.4142 & 1.3499 \\
50 & 1.4262 & 1.4142 & 1.3914 \\
\hline
\end{tabular}

Tabela 8.1: Valores da constante para A (3.15). 


\begin{tabular}{|c|ccc|}
\hline \multirow{2}{*}{$n_{1}=n_{2}$} & \multicolumn{3}{|c|}{$C_{\mathrm{r}} 2^{1 / 2}$} \\
\cline { 2 - 4 } & $1 / 2$ & 1 & $3 / 2$ \\
\hline \hline 10 & 1.4928 & 1.4142 & 1.3499 \\
50 & 1.4386 & 1.4142 & 1.3914 \\
50 & 1.4286 & 1.4142 & 1.4003 \\
\hline
\end{tabular}

Tabela 8.2: Valores da constante para A (3.31).

\begin{tabular}{|c|ccc|}
\hline \multirow{2}{*}{$n_{1}=n_{2}$} & $1 / 2$ & $C_{r} 2^{1 / 2}$ \\
\cline { 2 - 4 } 10 & 1.4928 & 1 & $3 / 2$ \\
\hline \hline 30 & 1.4386 & 1.4142 & 1.3499 \\
50 & 1.4286 & 1.4142 & 1.3914 \\
& & 1.4003 \\
\hline
\end{tabular}

Tabela 8.3: Valores da constante para A (4.16). 


\section{Bibliografia}

ABRAMOWITZ, M.; STEGUN, I.A. (1965). Handbook of Mathematical Functions with Formulas, Graphs and Mathematical Tables. Dover Publications, Inc., New York.

AITKIN, M. (1991). Posterior Bayes Factors. J. R. Statist. Soc. B, 53, 1, 111-142.

AKAIKE, H. (1973). Information Theory and an Extension of the Maximum Likelihood Principle. 2nd International Symposium on Information Theory. Budapest: Akademia Kiado, 267-281.

ATKInSON, A.C. (1978). Posterior Probabilities for Choosing a Regression Model. Biometrika, 65, 39-48.

BANSAL, N. (1987). A Confidence Region for the Slope Parameters in Errors in Variable Model. ASA Proceedings of the Business and Economic Statistics Section, 195-197.

BANSAL, N. (1990). Comparison of the Slope Parameters in Errors-inVariables Model. Comm. Statist. - Theory Meth., 19, 7, 2705-2716.

BERGER, J.O. ; PERICCHI, L.R. (1993). The Intrinsic Bayes Factor por 
Model Selection and Prediction. Technical Report, 83-43C.

BOLFARINE, H.; NASCIMENTO, J.A.; RODRIGUES, J. (1991). On the Estimation of the True $x$ Values in a Simple Regression Model when Both Variables have Error. $R T-M A E-9117$.

BOLFARINE, H.; NASCIMENTO, J.A.; RODRIGUES, J. (1992). Comparing Several Regression Models With Measurement Errors. A Bayesian Approach. RT-MAE-9201, IMEUSP.

BOLFARINE, H.; RODRIGUES, J.; CORDANI, L.K. (1992). O Modelo de Regressão com Erros nas Variáveis. $A B E-10^{\circ}$ SINAPE.

BOX, G.E.P.; HILL, W.H. (1967). Discrimination Among Mechanistics Models. Technometrics, 9, 57-71.

BOX, G.E.P.; TIAO, G.C. (1973). Bayesian Inference in Statistical Analysis. New York: Addison - Wesley.

CASELA, G. ; BERGER, R.L. (1987) Reconciling Bayesian and Frequentist Evidence in the One-Sided Testing Problem. J. Am. Statist. Ass., 82, 106-111

COX, D.R.; REID, N. (1987). Parameter Orthogonality and Approximate Conditional Inference. J.R.Statist. Soc. B, 49, 1, 1-39.

CREASY, M.A. (1956). Confidence Limits for the Gradient in Linear Functional Relationships. J.R.Statist. Soc. B, 18, 65-69.

FULLER, W. (1987). Measurement Error Models. New York, Wiley. 
GLEJSER, H. (1969). A New Test for Heterocedasticity. J. Am. Statist. Ass., 54, 173-205.

GOOD, I.J. (1947/50). Probability and the Weighting of Evidence. London: Griffin. New York: Haffner.

JEFFREYS, H. (1939, 1948, 1961). Theory of Probability . Oxford: University Press. ( $1 \underline{\underline{a}}, 2^{\underline{a}}$ e $3^{\underline{a}}$ eds. )

KASS, R.E.; TIERNEY, L.; KADANE, J.B. (1990). Laplace's Method in Bayesian Analysis. Unpublished report.

LINDLEY, D.V. (1957). A Statistical Paradox. Biometrika, 44, 187-192.

NELDER, J.A.; WEDDERBURN, R.W.M. (1972). Generalized Linear Models. J.R. Statist. Soc. A, 135, 370-384.

RODRIGUES, J.; BOLFARINE, H.; LOUZADA-NETO, F. (1993). Comparing Several Accelerated Life Models. Comm. Statist. - Theory Meth., 22, 8, 2297- 2308.

RODRIGUES, J.; CORDANI, L.K. (1990). A Note on Likelihood Estimation of a Simple Regression Model with Measurement Error. Via the Orthogonal Parametrization. South African Statist. J. 24, 177-183.

SCHWARZ, G. (1978). Estimating the Dimension of a Model. Ann. Statist. 6, 461-464.

SEBER, G. (1977). Linear Regression Analysis. New York, Wiley.

SHAFER, G. (1982). Lindley's Paradox. J. Am. Statist. Ass., 77, 325-351. 
SHANNON, C.E. (1949). "The Mathematical Theory of Comunication." Bell System Technical Journal, July-October 1948. Reprinted in C.E. Shannon and W. Weaver, The Mathematical Theory of Communication. University of Illinois Press, 3-91.

SMITH, A.F.M.; SKENE, A.M.; SHAW, J.E.H.; NAYLOR, J.C.; DRANSFIELD, M. (1985). The Implementation of the Bayesian Paradigm. Communs Statist. A, 14, 1079-1102.

SMITH, A.F.M.; SPIEGELHALTER, D.J. (1980). Bayes Factors and Choice Criteria for Linear Models. J.R.Statist. Soc. B, 42, 213-220.

SMITH, A.F.M.; SPIEGELHALTER, D.J. (1982). Bayes Factors for Linear and Log-linear Models with Vague Prior Information. J.R.Statist. Soc. B, 44, 377-387.

SPRENT, P. (1990). Some Historical of Functional and Structural Relationships. Contemporary Mathematics.

STONE, M. (1977). An Asymptotic Equivalence of Choice of Model by Cross-Validation and Akaike's Criterion. J.R. Statist. Soc. B, 39, 44-47.

VIEIRA, A.J.C. (1992). Construção e Caracterização de um Sensor de Altas Correntes Utilizando Fibras Opticas. São Carlos, SEL-EESC-USP

WONG, M.Y. (1991). Bartlett Adjustment to the Likelihood Ratio Statistic for Testing Several Slopes. Biometrika, 78, 221-224.

ZELLNER, A. (1971). An Introduction to Bayesian Inference in Econometrics. New York, Wiley. 
ZELLNER, A. (1984). Maximal Data Information Prior Distribuitions. Bayesian Econometrics and Statistics, 201-215. 